\title{
An Overview of the Dwarf Galaxy Survey
}

\author{
S. C. Madden ${ }^{1}$, A. Rémy-Ruyer ${ }^{1}$, M. Galametz ${ }^{2}$, D. Cormier ${ }^{1}$, V. Lebouteiller ${ }^{1}$, \\ F. Galliano ${ }^{1}$, S. Hony ${ }^{1}$, G. J. Bendo ${ }^{3}$, M. W. L. Smith ${ }^{4}$, M. Pohlen ${ }^{4}$, H. Roussel ${ }^{5}$, \\ M. Sauvage ${ }^{1}$, R. $\mathrm{Wu}^{1}$, E. Sturm ${ }^{6}$, A. Poglitsch ${ }^{6}$, A. Contursi ${ }^{6}$, V. Doublier ${ }^{6}$, M. Baes ${ }^{7}$, \\ M. J. Barlow ${ }^{8}$, A. Boselli ${ }^{9}$, M. Boquien ${ }^{9}$, L. R. Carlson ${ }^{10}$, L. Ciesla ${ }^{9}$, A. Cooray ${ }^{11}{ }^{12}$, \\ L. Cortese ${ }^{13}$, I. De Looze ${ }^{7}$, J. A. Irwin ${ }^{14}$, K. Isaak ${ }^{15}$, J. Kamenetzky ${ }^{16}$, \\ O. Ł. Karczewski ${ }^{8}$, N. Lu ${ }^{19}$, J. A. MacHattie ${ }^{14}$ B. O'Halloran ${ }^{17}$, T. J. Parkin ${ }^{18}$, \\ N. Rangwala ${ }^{16}$, M. R. P. Schirm ${ }^{18}$, B. Schulz ${ }^{19}$, L. Spinoglio ${ }^{20}$, M. Vaccari ${ }^{21}{ }^{22}$, \\ C. D. Wilson ${ }^{17}$ and H. Wozniak ${ }^{23}$
}




\footnotetext{
${ }^{1}$ Laboratoire AIM, CEA, Université Paris VII, IRFU/Service d'Astrophysique, Bat. 709, 91191 Gif-sur-Yvette, France

${ }^{2}$ Institute of Astronomy, University of Cambridge, Madingley Road, Cambridge CB3 OHA, UK

${ }^{3}$ UK ALMA Regional Centre Node, Jodrell Bank Centre for Astrophysics, School of Physics \& Astronomy, University of Manchester, Oxford Road, Manchester M13 9PL, UK

${ }^{4}$ School of Physics \& Astronomy, Cardiff University, The Parade, Cardiff, CF24 3AA, UK
}

${ }^{5}$ Institut d'Astrophysique de Paris, UMR7095 CNRS, Université Pierre \& Marie Curie, 98 bis Boulevard Arago, 75014 Paris, France

${ }^{6}$ Max-Planck-Institute for Extraterrestrial Physics (MPE), Giessenbachstrasse 1, 85748 Garching, Germany

${ }^{7}$ Sterrenkundig Observatorium, Universiteit Gent, Krijgslaan 281 S9, B-9000 Gent, Belgium

${ }^{8}$ Department of Physics and Astronomy, University College London, Gower St, London WC1E 6BT, UK

${ }^{9}$ Laboratoire d'Astrophysique de Marseille - LAM, Université d'Aix-Marseille \& CNRS, UMR7326, 38 rue F. Joliot-Curie, 13388 Marseille Cedex 13, France

${ }^{10}$ Leiden Observatory, Leiden University, Leiden, The Netherlands

${ }^{11}$ Center for Astrophysics and Space Astronomy, University of Colorado, 1255 38th Street, Boulder, CO, 80303, USA

${ }^{12}$ Division of Physics, Astronomy and Mathematics, California Institute of Technology, Pasadena, CA 91125 USA

${ }^{13}$ European Southern Observatory, Karl Schwarzschild Str. 2, 85748 Garching bei München, Germany

${ }^{14}$ Department of Physics, Engineering Physics \& Astronomy, Queen's University, 
Received

accepted

Kingston, ON K7L 3N6, Canada

${ }^{15}$ ESA Research and Scientific Support Department, ESTEC/SRE-SA, Keplerlaan 1, 2201 AZ Noordwijk, The Netherlands

${ }^{16}$ Department of Physics and Astronomy, University of California, Irvine, CA 92697 USA

${ }^{17}$ Astrophysics Group, Imperial College, Blackett Laboratory, Prince Consort Road, London SW7 2AZ, UK

${ }^{18}$ Department of Physics and Astronomy, McMaster University, Hamilton, ON L8S 4M1 Canada

${ }^{19}$ Infrared Processing and Analysis Center, Mail Code 100-22, California Institute of Technology, Pasadena, CA 91125, USA

${ }^{20}$ Istituto di Astrofisica e Planetologia Spaziali, INAF-IAPS, Via Fosso del Cavaliere 100, I-00133 Roma, Italy

${ }^{21}$ Astrophysics Group, Physics Department, University of the Western Cape, Private Bag X17, 7535, Bellville, Cape Town, South Africa

${ }^{22}$ Dipartimento di Astronomia, Università di Padova, vicolo Osservatorio, 3, 35122 Padova, Italy

${ }^{22}$ Observatoire astronomique de Strasbourg, Université de Strasbourg, CNRS, UMR 7550, 11 rue de lUniversité , F-67000 Strasbourg, France 


\begin{abstract}
The Dwarf Galaxy Survey (DGS) program is studying low-metallicity galaxies using 230h of far-infrared (FIR) and submillimetre (submm) photometric and spectroscopic observations of the Herschel Space Observatory and draws to this a rich database of a wide range of wavelengths tracing the dust, gas and stars. This sample of 50 galaxies includes the largest metallicity range achievable in the local Universe including the lowest metallicity $(\mathrm{Z})$ galaxies, $1 / 50 Z_{\odot}$, and spans 4 orders of magnitude in star formation rates. The survey is designed to get a handle on the physics of the interstellar medium (ISM) of low metallicity dwarf galaxies, especially on their dust and gas properties and the ISM heating and cooling processes. The DGS produces PACS and SPIRE maps of low-metallicity galaxies observed at 70,100, 160, 250, 350, and $500 \mu \mathrm{m}$ with the highest sensitivity achievable to date in the FIR and submm. The FIR fine-structure lines, [CII] $158 \mu \mathrm{m},[\mathrm{OI}] 63 \mu \mathrm{m},[\mathrm{OI}] 145 \mu \mathrm{m},[\mathrm{OIII}] 88 \mu \mathrm{m},[\mathrm{NIII}] 57 \mu \mathrm{m}$ and [NII] 122 and $205 \mu \mathrm{m}$ have also been observed with the aim of studying the gas cooling in the neutral and ionized phases. The SPIRE FTS observations include many CO lines $(\mathrm{J}=4-3$ to $\mathrm{J}=13-12)$, [NII] $205 \mu \mathrm{m}$ and [CI] lines at 370 and $609 \mu \mathrm{m}$. This paper describes the sample selection and global properties of the galaxies, the observing strategy as well as the vast ancillary database available to complement the Herschel observations. The scientific potential of the full DGS survey is described with some example results included.
\end{abstract}

Subject headings: galaxies: ISM - galaxies:dwarf- galaxies:NGC 4214- ISM: dust submillimeter: galaxies 


\section{Introduction}

One of the keystones in understanding how galaxies form and evolve is establishing the role of dwarf galaxies. Hierarchical models put dwarf galaxies forward as crucial building blocks of much larger galaxies, yet there is indication that many dwarf galaxies in our local Universe have stellar populations much younger than their larger cousins (e.g. Maiolino et al. 2008). A major source of this ambiguity may arise from the fact that accurate measurements to conclude on the properties of the Interstellar Medium (ISM) and star formation histories of local Universe low metallicity dwarf galaxies have been lacking, thus preventing a consistent picture to explain the interplay between the evolution of the metals and the process of star formation. How genuinely young galaxies harboring no old stellar population are related, if at all, to chemically young galaxies, is not at all clear. The scenery is quickly evolving, given the explosion of new wavelength coverage as well as spatial resolution and sensitivity provided by the current far-infrared (FIR) and submillimetre (submm) Herschel Space Observatory, which has made it possible to study in detail the vast variefof dwarf galaxies in our local Universe. These convenient laboratories allow us to study star formation and ISM properties in conditions that may be representative of early Universe environments, such as primordial galaxies where initial bursts of star formation may be taking place.

Before Herschel, much of what we have gleaned of the details of dust and gas properties and the processes of dust recycling, heating, and cooling in galaxies has been limited mostly to Galactic studies. ISO and Spitzer space missions have proven successful in demonstrating the importance of mid-infrared (MIR) to FIR observations to study the ionized and photodissociated gas emission measures and the unusual dust properties in the low luminosity, low metallicity galaxies (e.g. Engelbracht et al. 2005; Madden et al. 2006; Wu et al. 2006; Engelbracht et al. 2008). Systematic statistical surveys, that include 
submm wavelengths, spanning a wide variety of galactic physical parameter space such as metallicity, gas mass fraction, star formation activity, infrared (IR) luminosity, can be used to analyse in detail the physical processes at play in the low metallicity gas and dust over galaxy-wide scales. Only with such studies can we understand the impact of galactic properties on processes controlling the evolution of galaxies. To this end, the Herschel Dwarf Galaxy Survey (HDGS; P.I. Madden; 144.7h) is a compilation of a SPIRE SAG2 guaranteed time (GT) key program plus SPIRE GT2 observations, using the PACS and SPIRE instruments onboard the Herschel Space Observatory to obtain 55 to $500 \mu \mathrm{m}$ photometry and spectroscopy of 48 dwarf galaxies in our local Universe, chosen to cover a broad range of physical conditions. Our larger Herschel sample also includes 85.5h of complementary spectroscopy of dwarf galaxies, including pointings in 2 more galaxies, the Small Magellanic Cloud (SMC) and Large magellanic Cloud (LMC), carried out with the PACS GT key program and GT2 program, SHINING, which is also surveying starbursts and AGNs (P.I. Sturm). The total observing time of our Herschel PACS GT plus SPIRE GT surveys dedicated to 50 dwarf galaxies from our HDGS and SHINING programs, is $230 \mathrm{~h}$ and is hereafter together called the DGS (Dwarf Galaxy Survey).

The DGS sample has also been observed with MIR photometry and spectroscopy with Spitzer (i.e. Bendo et al. 2012b). Thus these data, together with the ancillary database being assembled, create a rich legacy for the community to perform dust and gas analyses in unprecedented detail in low metallicity galaxies from ultraviolet (UV) to millimetre (mm) wavelengths. The properties of the DGS makes it ideally suited for such studies (see section 3). Total infrared luminosity $\left(L_{T I R}\right)$ values of the survey range from $6.6 \times 10^{7} L_{\odot}$ for UGC4483, to our most luminous target, Haro $11, L_{T I R}=1.6 \times 10^{11} L_{\odot}$. Typical star formation rates range over 4 orders of magnitude $\left(0.0007\right.$ to $\left.28 M_{\odot} \mathrm{yr}^{-1}\right)$, with many of the dwarf galaxies containing super star clusters. Their metallicity properties span the largest range achievable in the local Universe, from a nearly solar value in He 2-10 to extremely low 
metallicity $\left(\right.$ ELM) environments with metallicity values, $12+\log (\mathrm{O} / \mathrm{H})<7.6\left(\sim 1 / 20 Z_{\odot}^{\top}\right)$ and ranging as low as $\sim 1 / 40$ to $1 / 50 Z_{\odot}$ in galaxies such as I Zw 18 and SBS 0335-052 among the most metal-poor in the local Universe. Furthermore, these objects have wide variations of gas mass fractions relative to stellar mass (0.001 to 0.7), dust-to-metal mass ratios (0.07 to 0.33$)$, and IR luminosities-to-gas mass ratios (0.4 to 20).

The DGS obtains detailed spatial information on the well-studied nearby star-forming dwarf galaxies of the Local Group. The well-resolved objects serve as anchors between the detailed local physical processes and the larger statistical sample. Size scales of a few pc to 100 pc allow for the detailed multiphase study of low metallicity ISM, where stellar winds and supernovae can have a violent impact on the ISM, controlling the evolution of the dust and gas properties in dwarf galaxies. The DGS includes PACS spectroscopic maps in several bright star forming regions in the $\mathrm{LMC}\left(1 / 2 Z_{\odot}\right)$ and the $\mathrm{SMC}\left(1 / 5 Z_{\odot}\right)$ which complement the study of HERITAGE - the Herschel FIR and submm photometric survey of the Magellanic Clouds (Meixner et al. 2010, Meixner et al 2013, submitted).

The DGS, along with ancillary data existing and continuing to be collected, provides the community with an unprecedented database of high impact legacy value and will lead to many future follow-up studies. To date, the DGS observations have been the objective of several case studies already presented or in preparation: NGC 6822 Galametz et al. 2010, Carlson et al. 2013, in preparation), NGC 1705 (O'Halloran et al. 2010), NGC 4214 (Cormier et al. 2010), Haro 11 (Cormier et al. 2012), LMC/N11B (Lebouteiller et al. 2012), NGC 4449 (Karczewski et al. 2013). The results of the full photometric and spectrometric DGS will be presented in Rémy-Ruyer et al. (2013a, in preparation) and Cormier et al. (2013, in preparation ), respectively. The utility of the FIR fine-structure lines as star

$$
\begin{aligned}
& { }_{1}^{1} \text { throughout we assume solar } \\
& 12+\log (\mathrm{O} / \mathrm{H})_{\odot}=8.7
\end{aligned}
$$


formation indicators and the dependence on metallicity will be presented in de Looze et al (2013, in preparation). The interpretation of this full database will lay the groundwork for understanding star formation activity under low metallicity ISM conditions, thus providing benchmarks against which primordial galaxies can be compared. Some of the outstanding questions which can be addressed with this database include the following:

The global properties and diagnostics of low metallicity galaxies

- What properties of the galaxy as a whole, and local processes within it, control the observed Spectral Energy Distribution (SED) and its evolution? How can we disentangle the effects of galaxy metallicity, star formation properties, ISM morphology and dust properties (dust size distribution, composition, etc.), etc on the observed SED?

- In which galactic environments does the submm exces: $2^{2}$ manifest and what is the nature of this submm excess?

- How can we most accurately trace the total molecular gas reservoirs which are fueling the prominent star formation activity in low metallicity dwarf galaxies? Is the warm molecular gas a significant component in star forming dwarf galaxies? Reliable CO-to- $\mathrm{H}_{2}$ conversion factors for low metallicity ISM conditions are urgently needed.

- How can we calibrate the technique of using observed dust masses to get at the gas reservoirs?

\footnotetext{
${ }^{2}$ The submm excess is emission enhanced beyond that explicable by current dust models and normally appears near or longward of $\lambda \geq 500 \mu \mathrm{m}$ in low metallicity environments (see Section 2).
} 
- Is the $158 \mu \mathrm{m}$ [CII] line a valid star formation rate tracer in local galaxies, and can it be used to quantify star formation in high- $z$ galaxies?

The impact of star formation on the ISM

- What are the effects of the ensemble of star formation sites on full galaxy scales and the processes controlling the feedback between the surrounding metal-poor ISM and star formation under conditions approaching those of the earliest, most metal-poor galaxies?

- What is the impact of the super star clusters on the nature of the surrounding dust and gas? How much star formation activity is actually enshrouded and optically thick out to and beyond MIR wavelengths even in these dust-poor environments?

- What is the effect of metallicity (direct or indirect) on the morphology and filling factors of the different gas phases?

The interplay between the gas and dust in galaxies

- What are the consequences of the dust properties on the gas heating and cooling processes in low metallicity environments and how does this compare with metal-rich ISM?

- What is the relationship between metals in the gas phase and those in the dust as a function of the metallicity of a galaxy? How do the dust-to-gas mass ratios $(\mathrm{D} / \mathrm{G})$ evolve as a function of galaxy metallicity?

- What is the impact of dust abundance and composition and gas reservoirs on the evolution of the ISM and vice versa? 
The improved angular resolution and sensitivity of Herschel in the 60-160 $\mu \mathrm{m}$ range compared to ISO and Spitzer, is allowing us to probe local regions within galaxies as well as statistically-important global galaxy properties. Thus we can study the effect of varying spatial scales on the interpretation of the observations and relate the observed variations in the dust SEDs and gas properties to the actual physical phenomena occurring within the galaxy (e.g. Bendo et al. 2012b; Parkin et al. 2012; Foyle et al. 2012; Aniano et al. 2012, Galametz et al. 2012; Fritz et al. 2012; Smith et al. 2012; Xilouris et al. 2012; Mentuch Cooper et al. 2012). The added benefit of the combination of the Herschel spectroscopy and photometry will make it possible to address the long-standing issues on the total gas and dust reservoirs in low metallicity galaxies, with particular attention to the enigma of quantifying the molecular gas reservoir. This will lead to the construction of a rich interpretative framework for unresolved, more distant galaxies in the early Universe.

In this paper we present the overview of the scientific goals in section 2 ; the source selection and properties are described section 3 , the observing strategy and data treatment of the DGS and ancillary data of the survey are described in section 4; selected science results are presented in section 5: future products to come out of the survey are mentioned in section 6, and a final summary is given in section 7 .

\section{Scientific Goals}

\subsection{The dust properties of dwarf galaxies}

One avenue to understand the star formation activity and the evolution of dwarf galaxies is by studying the dust emission properties. In addition to being the culprit in obscuring the starlight in galaxies, potentially biasing our view of stellar activity, dust plays a more positive role as a major actor in the thermal equilibrium of galaxies. Dust absorbs 
starlight of a wide range of energies, emitting in the MIR to submm/mm wavelength regime. In spite of their metal-poor ISM, dwarf galaxies have been known, even since IRAS observations, to possess non-negligible amounts of dust with some low metallicity galaxies globally harboring warmer dust on average than their dust-rich counterparts. The more active blue compact dwarf galaxies (BCDs), for example, revealed higher IRAS 60/100 $\mu \mathrm{m}$ flux ratios (i.e. warmer dust) compared to those of disk-type metal-rich galaxies while the elevated $25 / 12 \mu \mathrm{m}$ fluxes highlighted the presence of warm stochastically-heated MIR-emitting dust and the dearth of the smallest grains/large molecules, Polycyclic Aromatic Hydrocarbons (PAHs) (e.g. Helou 1986; Hunter et al. 1989; Sauvage et al. 1990; Melisse \& Israel 1994). The dramatic improvement in sensitivity of Spitzer accentuated these findings of galaxy-wide warmer dust in star forming dwarf galaxies (e.g. Jackson et al. 2006; Rosenberg et al. 2006; Cannon et al. 2006; Walter et al. 2007), with an extreme case being one of the lowest metallicity galaxies known in the Universe, SBS0335-052 Houck et al. 2004). ISO and Spitzer spectroscopy confirmed the relatively weak PAH bands in dwarf galaxies (e.g. Madden et al. 2006, O’Halloran et al. 2006; Wu et al. 2006; Hunter \& Kaufman 2007; Smith et al. 2007; Hunt et al. 2010) and are sometimes completely absent as metallicities drop to a threshold level of about $12+\log (\mathrm{O} / \mathrm{H})=8.2-$ about $1 / 5$ to $1 / 3$ $Z_{\odot}($ Engelbracht et al. 2005).

While observations have been modeled with sufficient MIR to submm coverage in only a limited number of local Universe metal-poor galaxies with IRAS, ISO, Spitzer and ground-based telescopes, their SEDs were already exhibiting evidence for dust properties with other notable differences from those of our Galaxy as well as other metal-rich starburst galaxies. Excess emission in the submm/mm wavelength range (Figure 11) over the expected Rayleigh-Jeans behavior was noted in the COBE observations of our Galaxy (Reach et al. 1995) and has been detected in low-metallicity dwarf galaxies using ground-based telescopes observing $850 / 870 \mu \mathrm{m}$ with SCUBA on the JCMT and with 
LABOCA on APEX (e.g. Galliano et al. 2003, 2005; Bendo et al. 2006, Galametz et al. 2009; Zhu et al. 2009; Galametz et al. 2011). In the LMC, where Herschel brings 10 pc resolution at $500 \mu \mathrm{m}$, Galliano et al. (2011) have highlighted locations of submm excess ranging from $15 \%$ to $40 \%$ compared to that expected from dust SED models. The intensity of the excess is anti-correlated with the dust mass surface density. Possible explanations put forth to explain the submm excess include: 1) very cold dust component (e.g. Galliano et al. 2005; Galametz et al. 2011); 2) unusual dust emissivity properties (e.g. Lisenfeld et al. 2002; Meny et al. 2007; Galliano et al. 2011; Paradis et al. 2011); 3) anomalous spinning dust which has explained excess emission appearing at radio wavelengths (e.g. Draine \& Lazarian 1998; Hoang et al. 2011; Ysard et al. 2011) and 4) magnetic dipole emission from magnetic grains (Draine \& Hensley 2012). No single explanation put forth to date as the origin of the submm excess is completely satisfactory for all cases observed. Thus, the origin of the submm excess remains enigmatic. With more sensitive submm wavelength coverage, Herschel is confirming the flatter submm slope indicative of the submm excess present in some dwarf galaxies in the DGS (i.e. Rémy-Ruyer et al. 2013a, in preparation).

The global MIR SEDs of dwarf galaxies, often dominated by prominent ionized gas lines, weak PAH emission and steeply rising MIR continuum, resemble HII regions (e.g. Madden et al. 2006), or even, to some extent, ULIRGs (Figure 1). Galaxy-wide, star forming dwarf galaxies often have a larger proportion of warm dust than spiral galaxies, with steeply rising MIR to FIR slopes and overall SEDs peaking at wavelengths shortward of $\sim 60 \mu \mathrm{m}$ (Figure 1) (e.g. Galametz et al. 2009, 2011). Starbursting dwarf galaxies have a higher relative abundance of small grains, compared to our Galaxy (e.g. Lisenfeld et al. 2002; Galliano et al. 2003, 2005). In one of the most metal-poor galaxies detected to date, SBS 0335-052 $\left(1 / 40 Z_{\odot}\right)$, the MIR to FIR SED is unlike that of any other galaxy observed so far, with silicate absorption bands superimposed on a featureless continuum peaking at an unusually short wavelengths, $\sim 30 \mu \mathrm{m}$ (Thuan et al. 1999; Houck et al. 2004). The 
smallest grains in galaxies, the most numerous in low metallicity galaxies being the very small grains, not the PAHs which are abundant in disk galaxies, are normally playing the most important role in gas heating, commonly via the photoelectric effect (Bakes \& Tielens 1994). Thus how the dust size distribution and abundance varies within galaxies is critical to understand the heating and cooling processes. Given these observed dust properties, the heating and cooling processes will, undoubtedly, be altered in dust-poor galaxies relative to dust-rich galaxies. The structure of the ISM in terms of relative filling factors of star clusters, atomic, ionized and molecular gas looks to be different from those in our Galaxy or other metal-rich starburst galaxies: the ionized regions and the galaxy-wide hard radiation field, play an important role in shaping the observed SED and the gas properties (Lebouteiller et al. 2012; Cormier et al. 2012). Simply assuming Galactic dust properties in the analyses of SEDs of low metallicity galaxies may lead to erroneous results in the determination of dust mass, extinction properties, gas mass, star formation properties, etc.

The choice of dust composition to use when interpreting the observations complicates the problem. For example, assuming the often-used graphite dust analog, which, along with a silicate and PAH components, well-describes the dust composition of the Galaxy (e.g. Zubko et al. 2004, Draine \& Li 2007) can lead to inexplicably large dust masses in low metallicity galaxies such as the LMC, as shown by Galliano et al. (2011). While either amorphous carbon or graphite and silicate and PAHs in the SED model can successfully fit the observations, the resulting larger dust mass using graphite, is sometimes difficult to reconcile with the metallicity, the observed gas mass and the expected $\mathrm{D} / \mathrm{G}$ (Section 2.4). Posessing higher emissivity properties, an amorphous carbon component (Zubko et al. 1996) can result in a lower dust mass that agrees better in some cases with the measured gas mass and the expected $\mathrm{D} / \mathrm{G}$. A recent reevaluation of the optical properties of (hydrogenated) amorphous carbon may lead to yet a different view of the variations in the observed dust

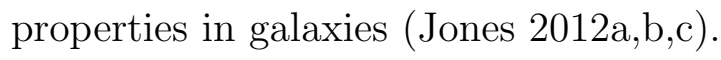




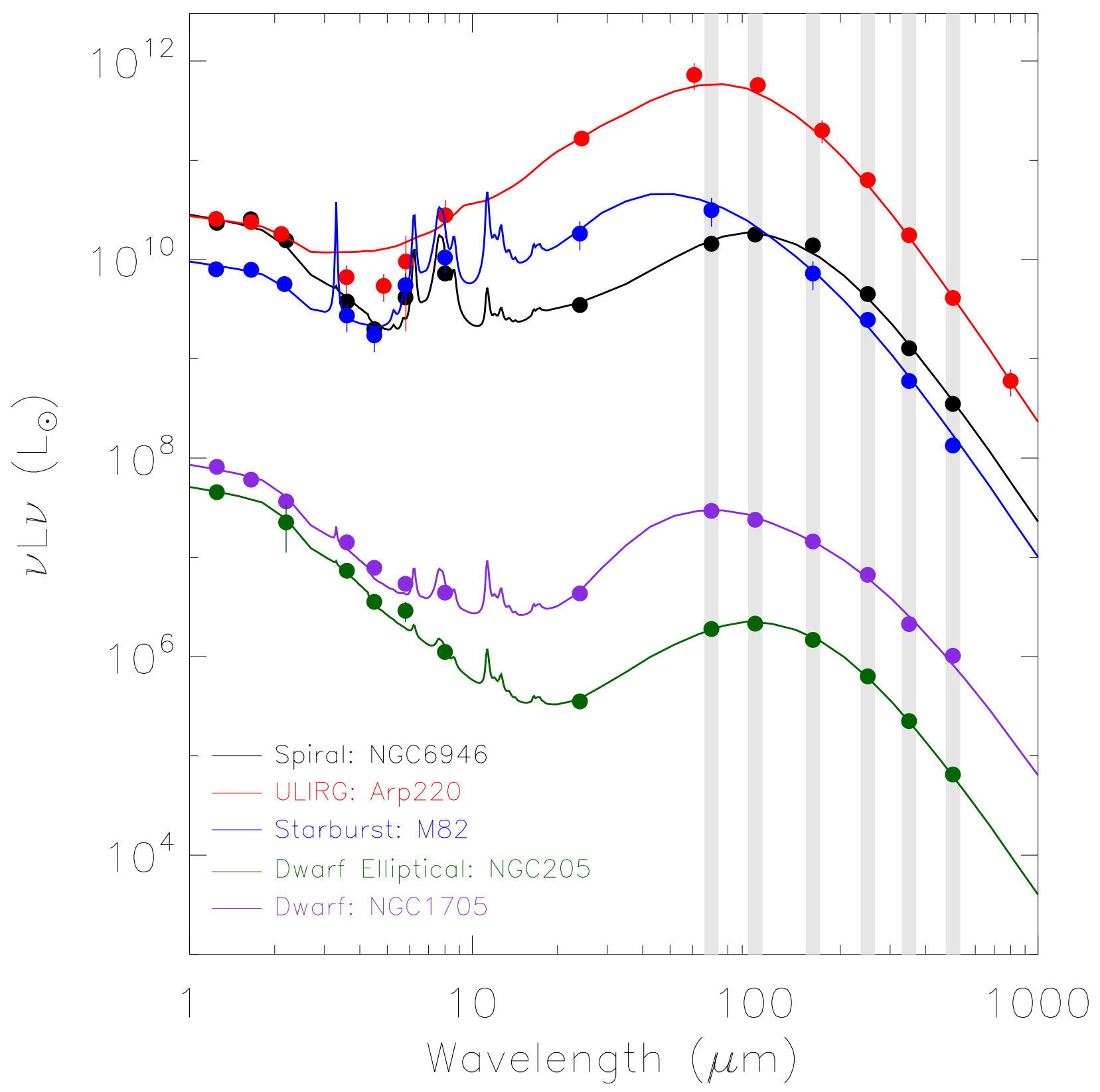

Fig. 1.- Comparison of a dwarf galaxy with the SEDs of 4 other galaxies: star-forming dwarf galaxy, NGC 1705 (O'Halloran et al. 2010) with a spiral galaxy, NCG 6946 Aniano et al. 2012), a Ultra Luminous InfraRed Galaxy (ULIRG), Arp 220, a metal-rich starburst, M82 (Roussel et al. 2010) and a dwarf elliptical, NGC 205 (De Looze et al.|2012). The dwarf galaxy, NGC 1705, is one of the faintest example here with a SED characteriztic of starforming low metallicity dwarf galaxies. The vertical bars indicate the Herschel PACS and SPIRE channels. The SED models are derived using the Galliano et al. "AC" (amorphous carbon) model (Galliano et al. 2011). 
Many of the conclusions on the dust properties of low metallicity ISM rest on the need for higher spatial resolution and longer wavelength coverage into the submm, as provided by Herschel. While Draine et al. (2007) concluded that there is little effect overall on the dust masses of galaxies with or without submm observations, Galametz et al. (2011) found that including submm constraints in the SED modelling process can affect the dust masses estimated. To complicate interpretation, the single temperature modified black body, often used to describe the dust FIR-submm properties, can lead to different dust masses, for

example, compared to more realistic dust SED models (e.g. Galliano et al. 2011; Dale et al. 2012; Galametz et al. 2012). This is a consequence of the fact that 70-500 $\mu \mathrm{m}$ emission from galaxies can originate from different heating sources and a single modified blackbody fitting these data can overestimate the average dust temperature and thus underestimate the dust mass within the galaxies (e.g. Bendo et al. 2010; Boquien et al. 2011; Bendo et al. 2012b; Dale et al. 2012). Herschel is well-positioned to lend clarity to these issues.

\subsection{FIR Fine-Structure lines as gas coolants in the interstellar medium}

The Kennicutt-Schmidt law demonstrates the empirical relationship between total gas column density and star formation rate surface density. The observed slope of $\sim 1.4$ indicates that the efficiency in forming stars increases with the gas surface density (Kennicutt 1998). Evidence is growing for molecular gas surface density, in particular, as the regulator for star formation in galaxies of the local Universe as well as at high-redshift, and not necessarily the total gas density (e.g. Kennicutt et al. 2007; Bigiel et al. 2008; Genzel et al. 2012). While mostly more massive galaxies have been used to derive this relationship, does the same recipe hold for star formation in the wide range of low mass dwarf galaxies, or, for that matter, high- $z$ low metallicity dwarf galaxies?

We take for granted that $\mathrm{CO}(\mathrm{J}=1-0)$ is the best tracer of molecular gas in local and 
distant galaxies, considering the observational difficulties in detecting directly the most abundant molecule, $\mathrm{H}_{2}$. While $\mathrm{HI}$ halos are often very extended in dwarf galaxies, CO has been notoriously difficult to detect. Traversing from CO ( J=1-0) observations to the total $\mathrm{H}_{2}$ gas mass in dwarf galaxies is a perplexing issue, one that has been hampering studies of low metallicity ISM for decades. This CO $(\mathrm{J}=1-0)$ deficit is often difficult to reconcile with the molecular gas necessary to fuel star formation and valiant effort has been invested in determining a recipe for the $\mathrm{CO}(\mathrm{J}=1-0)$-to- $\mathrm{H}_{2}$ conversion factor in low metallicity galaxies (e.g. Leroy et al. 2011; Schruba et al. 2012; Bigiel et al. 2011, and references within). On the other hand, surprisingly prominent FIR fine structure cooling lines, such as the 158 $\mu \mathrm{m}[\mathrm{CII}]$ line, often interpreted as the best tracer of photodissociation regions (PDRs) around molecular clouds illuminated by massive stars, have been detected in low metallicity dwarf galaxies with the Kuiper Airborne Observatory (KAO) and the ISO LWS Stacey et al. 1991; Poglitsch et al. 1995; Madden et al. 1997; Madden 2000; Bergvall et al. 2000; Hunter et al. 2001; Vermeij et al. 2002; Brauher et al. 2008; Israel \& Maloney 2011). Thus, exceptionally bright $158 \mu \mathrm{m}[\mathrm{CII}]$ with exceptionally faint $\mathrm{CO}(\mathrm{J}=1-0)$ renders the ISM of low metallicity galaxies and the conditions for star formation rather elusive.

$\mathrm{L}_{[C I I]}$ has been hailed as an important tracer of star formation activity (Stacey et al. 1991; Boselli et al. 2002; de Looze et al. 2011). For example, values of $\mathrm{L}_{[C I I]} / \mathrm{L}_{C O(J=1-0)}$ of more active normal starburst galaxies have ratios of $\sim 4000$ while more quiescent spiral galaxies show a factor of 2 lower (Stacey et al. 1991). In dwarf galaxies, however, values of $\mathrm{L}_{[C I I]} / \mathrm{L}_{C O(J=1-0)}$ can range from about 4000 to as high as $\sim 80000$ (Madden 2000) much higher than the most active metal-rich galaxies (Figure 2). What is the explanation behind such strikingly high $\mathrm{L}_{[C I I]} / \mathrm{L}_{C O(J=1-0)}$ ?

Toward regions of the Local Group galaxy, IC10, for example, [CII] excitation from collisions with $\mathrm{H}$ atoms could not explain the surprisingly high observed [CII] intensity. 
Assuming the $[\mathrm{CII}]$ excitation originated in PDRs, comparison of the observed HI column density required an additional excitation source, namely $\mathrm{H}_{2}$ molecules, to reconcile the cooling rate with the photoelectric UV heating rate. This lead to the suggestion that up to 100 times more molecular hydrogen gas may be present, compared to the $\mathrm{H}_{2}$ inferred from the relatively faint CO ( $\mathrm{J}=1-0)$ lines (Madden et al. 1997). These early KAO detections of the $158 \mu \mathrm{m}$ [CII] line in low metallicity galaxies, namely the LMC (Poglitsch et al. 1995; Israel et al. 1996; Israel \& Maloney 2011) and IC10 (Madden et al. 1997) were the first observational evidence for the presence of a reservoir of $\mathrm{H}_{2}$ gas not traced by $\mathrm{CO}(\mathrm{J}=1-0)$, but residing in the $\mathrm{C}^{+}$-emitting region - the "CO-dark" molecular gas.

The presence of the CO-dark molecular gas is regulated by conditions present in low metallicity environments. Molecules which are not effective at self-shielding, such as CO, will be photo-dissociated before $\mathrm{H}_{2}$ in the PDRs. The combined effect of the strong FUV field from the nearby young star clusters and the decreased dust abundance is to shift the atomic-molecular gas transition deeper into the cloud, reducing the relative size of the $\mathrm{CO}$ core and increasing the volume of the PDR. In this scenario, a potentially significant reservoir of $\mathrm{H}_{2}$ can be present in $\mathrm{C}^{+}$- emitting PDR, outside the smaller $\mathrm{CO}$ core. This component, not traced by CO nor HI, has also been uncovered in our Galaxy via $\gamma$ - ray observations (Grenier et al. 2005) and more recently with Planck (Planck Collaboration et al. 2011; Paradis et al. 2012). This effect of the CO-dark molecular gas in the PDR envelope is shown theoretically to be controlled strongly by $A_{V}$ and should increase in importance as the metallicity decreases (Wolfire et al. 2010; Krumholz \& Gnedin 2011; Glover \& Clark 2012). More recent detailed studies comparing the dust and observed gas properties in the LMC and other nearby galaxies with Herschel and Planck have likewise highlighted the presence of this otherwise undetected gas phase (Section 2.4), which could also be in the form of optically thick HI. 


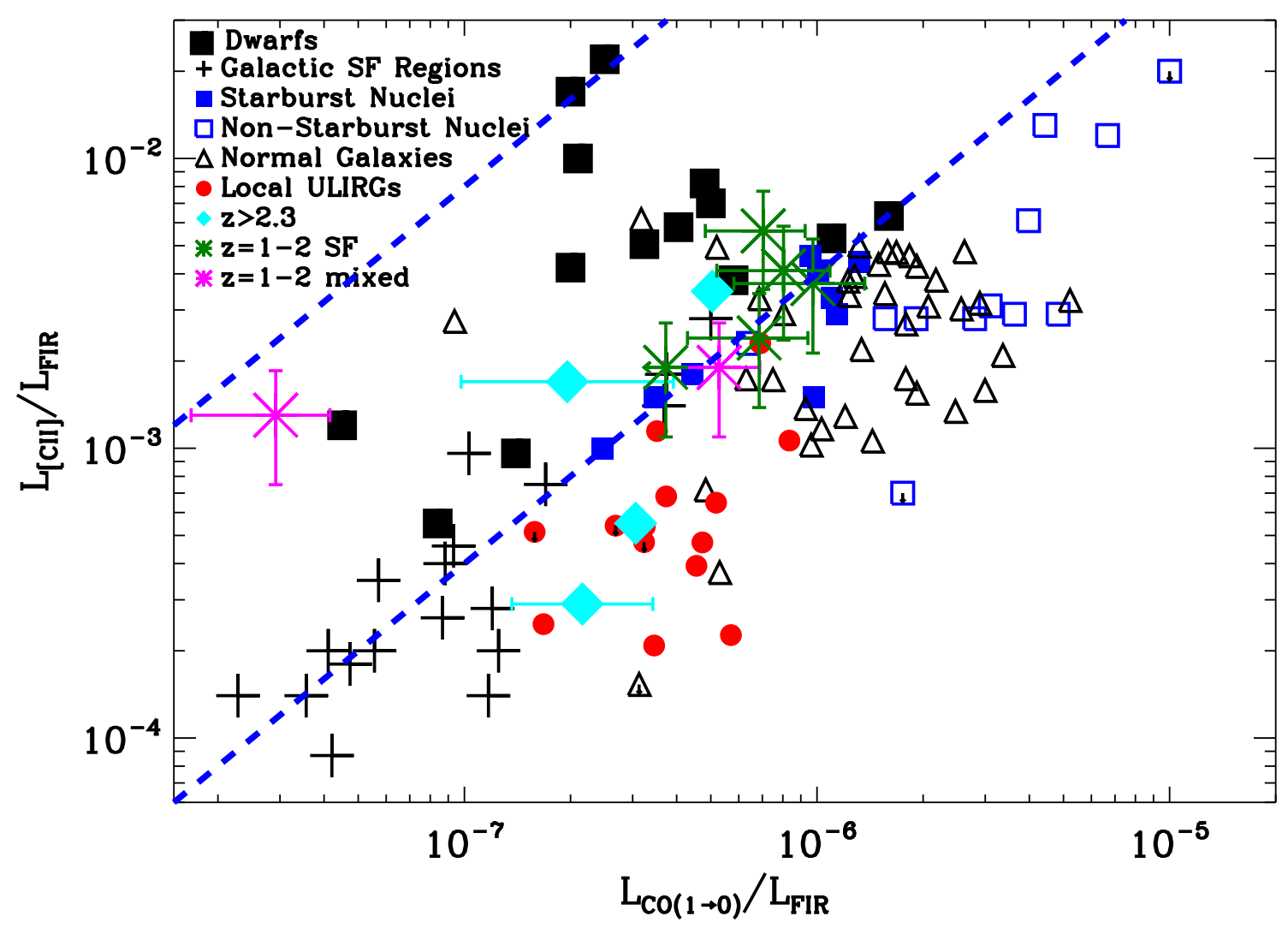

Fig. 2.- $\mathrm{L}_{[C I I]} / \mathrm{L}_{F I R}$ vs $\mathrm{L}_{C O} / \mathrm{L}_{F I R}$ for local galaxies, and high- $z$ galaxies (Hailey-Dunsheath et al. 2010; Stacey et al. 2010) and low metallicity dwarf galaxies (Madden 2000). Lines of constant $\mathrm{L}_{[C I I]} / \mathrm{L}_{C O}$ are shown (blue, dashed) for 4,000 (local starburst and high- $z$ galaxies) and 80,000, the maximum end of dwarf galaxies. These observations are from the ISO/LWS and the KAO. Herschel observations of dwarf galaxies will populate this plot further to the upper left region. 
In addition to the widely-used [CII] line, the DGS spectroscopy programme traces the other key FIR ISM cooling lines such as [OIII], [OI], [NIII] and [NII] (Table 1). These important cooling lines are diagnostics to probe the FUV flux, the gas density (n) and temperature $(\mathrm{T})$ and the filling factor of the ionized gas and photodissociation regions (e.g. Wolfire et al. 1990; Kaufman et al. 2006). While the new NASA-DFG airborne facility, SOFIA, will continue observing the FIR fine-structure lines beyond Herschel's lifetime, it must contend with the residual effects of the Earth's atmosphere. Hence, Herschel allows us the best sensitivity and spatial resolution to probe a variety of emission lines from these intrinsically faint dwarf galaxies.

Since the ionization potential of $\mathrm{C}^{0}$ is $11.3 \mathrm{eV}$ (Table 1) - less than that required to ionize atomic hydrogen - [CII] can be found in the ionized as well as the neutral phases. One way to determine how much of the observed [CII] may be excited by collisions with electrons in low density ionized regions, for example, is to use the [NII] lines at 122 and 205 $\mu \mathrm{m}$ (e.g. Oberst et al. 2011). Since $\mathrm{N}^{0}$ requires an ionization potential of $14.5 \mathrm{eV}$, these lines arise unambiguously from a completely ionized phase. Their relatively low critical densities $\left(n_{\text {crit }}\right)$ for collisions with electrons $\left(48\right.$ to $\left.200 \mathrm{~cm}^{-3}\right)$ make them useful to associate any observed [CII] emission possibly arising from the diffuse ionized gas, and thus, provide a means to extract the PDR-only origin component of the [CII] emission. The [NII] $122 \mu \mathrm{m}$ line is detected with PACS only in the brightest regions of the DGS galaxies. The PACS [NII] $205 \mu \mathrm{m}$ line was not observable in the dwarf galaxies: leakage from other orders of the grating renders the flux calibration of the [NII] $205 \mu \mathrm{m}$ line unreliable and the sensitivity of the instrument beyond $190 \mu \mathrm{m}$ is degraded. The [NII] $205 \mu \mathrm{m}$ line is, however, covered by the SPIRE FTS which has observed 4 DGS galaxies. The [OIII] $88 \mu \mathrm{m}$ line has a similarly low $n_{\text {crit }}$ for collisions with electrons (Table 1) and can also be associated with the low density ionized medium. It has the highest ionization energy of these FIR lines and traces the hard photons traversing the diffuse ionized medium. Thus, with Herschel, along with 
the existing ancillary data, we can more accurately model the diffuse and dense atomic, ionized and molecular phases of the low-metallicity ISM.

The sensitivity provided by Herschel, along with the wide range of MIR lines that have been observed for the target galaxies with the Spitzer IRS spectrograph (Table 5), provides $\sim 20 \mathrm{MIR}$ and FIR fine structure lines in a wide variety of low metallicity galaxies in the DGS. Detailed studies of the photodissociated and photoionized gas can characterize the physical properties of the ISM as well as the properties of the energetic sources in low metallicity environments (see Cormier et al. (2012) for an example of such complex extragalactic modeling). Additionally, these observations will be able to shed light on quantifying the molecular gas reservoir in dwarf galaxies.

\subsection{Possible Warm Gas Reservoir in Dwarf Galaxies?}

MIR $\mathrm{H}_{2}$ lines, possible tracers or warm gas, can sometimes be present in Spitzer IRS spectra of dwarf galaxies, most often at low levels (Wu et al. 2006; Hunt et al. 2010), but are not normally thought to account for a substantial reservoir of the molecular gas in dwarf galaxies because most of this gas lies at lower $\mathrm{T}(<100 \mathrm{~K})$.

The SPIRE FTS, covering 194 to $670 \mu \mathrm{m}$, provides simultaneous coverage of CO rotational lines ranging from $\mathrm{J}=4-3$ through $\mathrm{J}=13-12$, a range within which the spectral line energy distribution (SLED) of CO lines has been found to peak for AGNs, star forming galaxies and for high-z QSOs unveiling, often, a previously unknown warm molecular component (e.g. Panuzzo et al. 2010; Rangwala et al. 2011; Kamenetzky et al. 2012; Spinoglio et al. 2012). The warm molecular gas component of the relatively bright, well-studied nearby dwarf galaxies, NGC 4214, IC10, He2-10 and 30 Doradus, the most massive star forming region in our neighboring LMC, are observed with the SPIRE FTS. 
Table 1. PACS FIR emission lines for the DGS: their properties and diagnostic capabilities

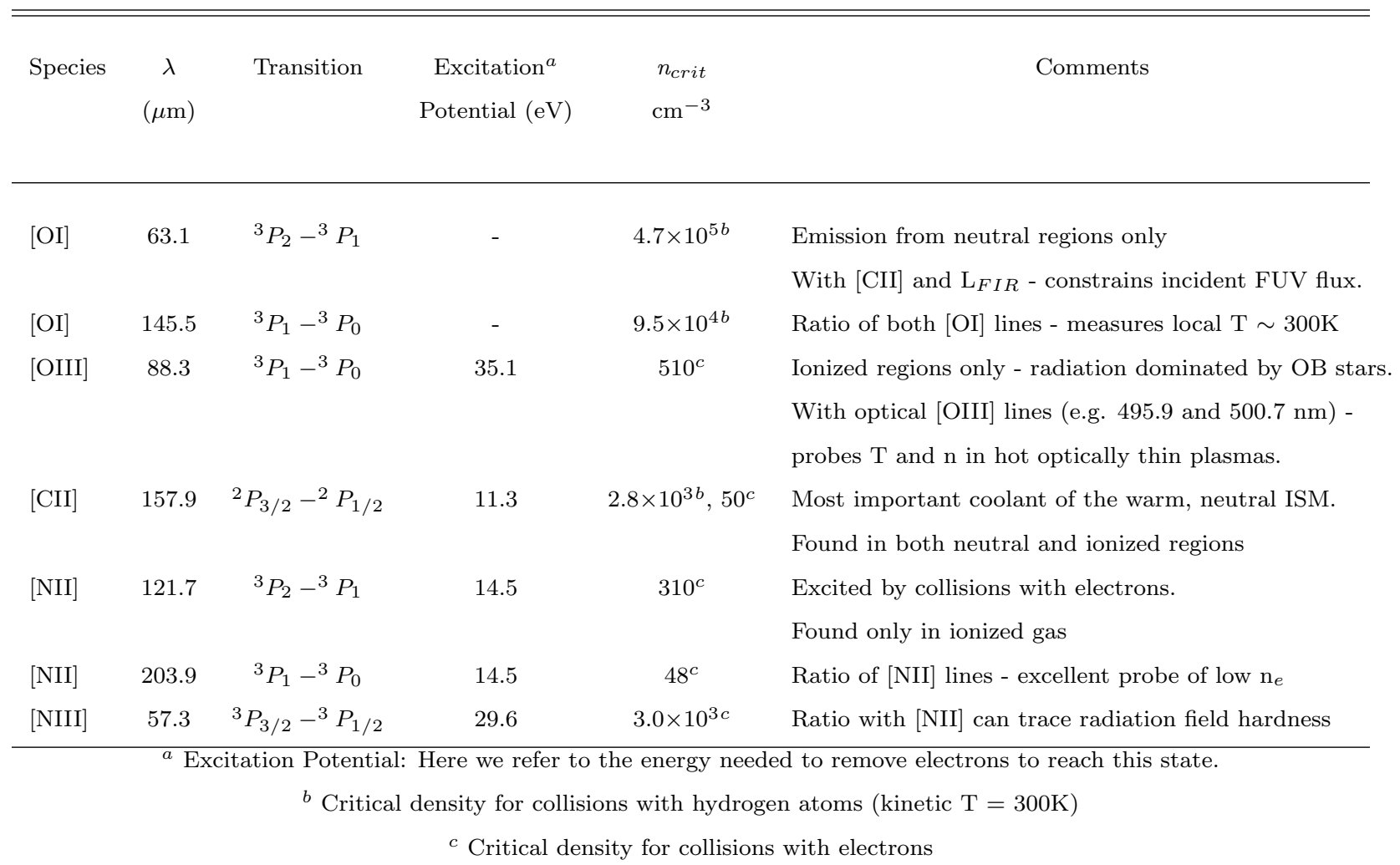


30Doradus, He2-10 and IC10 have already been detected from the ground in CO transitions as high as $\mathrm{J}=7-6$ (Bayet et al. 2004, 2006; Pineda et al. 2012). While CO J=1-0 can be excited at low densities $\left(10^{2}\right.$ to $\left.10^{3} \mathrm{~cm}^{-3}\right)$ and low temperatures $(\sim 10 \mathrm{~K}-50 \mathrm{~K})$, higher-J CO lines effectively trace the denser and warmer gas connected to ongoing star-formation. The high $\mathrm{J}$ ground-based CO lines (up to $\mathrm{J}=7-6$ ) have been touted to be the best available SFR indicators accessible with ground-based instruments (Bayet et al. 2009). The FTS observations, aided by PDR and chemical models, will lead to the determination of the physical parameters (including density, temperature, filling factor and mass) of the CO-emitting molecular reservoir in low metallicity galaxies. This warm component is essential to help provide a complete inventory of all of the gas in these star-forming low metallicity galaxies, much of it possibly residing in the warmest reservoirs more reliably traced by the high-J CO lines from the FTS observations.

\subsection{Dust to Gas Mass ratios (D/G)}

Limits in sensitivity, spatial resolution and wavelength coverage in the past have left an ambiguity in the behavior of the D/G at low metallicities (e.g. Galametz et al. 2011), The DGS is finally extending studies on $\mathrm{D} / \mathrm{G}$ in galaxies to the lowest metallicities in the local Universe (Rémy-Ruyer et al. 2013b in preparation).

While the proportion of heavy elements in the gas and dust scales with the metallicity in the disk of our Galaxy (Dwek 1998), it is not completely clear that this also holds for low metallicity dwarf galaxies. For a sample of low-metallicity galaxies, Lisenfeld \& Ferrara (1998) and Issa et al. (1990) found that the dependence of metallicity on D/G can deviate from the expected linear relationship which implies that the fraction of metals in the dust phase varies over the history of a galaxy. These studies, however, were performed for dust masses determined from IRAS observations, therefore not tracing cooler dust 
beyond $100 \mu \mathrm{m}$. James et al. (2002), who did a similar study using some ground-based $850 \mu \mathrm{m}$ observations, include a few low metallicity galaxies in their large sample and find a flatter trend of the $\mathrm{D} / \mathrm{G}$ at lower metallicities that needs to be tested for a larger range of metallicities, particularly for lower metallicities. In a study conducted before Herschel, Galametz et al. (2011) demonstrated that submm wavelength observations were indispensable to constrain the total dust masses, leaving some ambiguity in the measured $\mathrm{D} / \mathrm{G}$ even without considering a submm excess. On the other hand, Draine et al. (2007) concluded that the D/G of the SINGS sample of galaxies was well determined with Spitzer observations up to $160 \mu \mathrm{m}$ with an additional cirrus component to explain the $850 \mu \mathrm{m}$ observations of the metal-rich galaxies in the sample. More recently, Dale et al. (2012) results extending the SINGS sample to Herschel wavelengths with the KINGFISH survey (Kennicutt et al. 2011), note a submm excess present in their low Z galaxies. Herschel is beginning to uncover more examples of possible candidates to study the submm excess as shown, for example, in the dwarf galaxies in the Herschel Virgo Cluster Survey Grossi et al. 2010). Likewise, a metallicity dependence of the SPIRE band ratios in the Herschel Reference Survey (Boselli et al. 2012) suggests the presence of a submm excess. More complete wavelength sampling by Herschel will help resolve the submm behavior and refine our extragalactic dust SED models.

Bot et al. (2004) modelled the dust in the diffuse ISM of the SMC ( $Z=1 / 10)$, and concluded that the $\mathrm{D} / \mathrm{G}$ is 30 times lower than that of the Galaxy - 3 times lower than the value a linear scaling of the metallicity would suggest. The SMC result supports the idea of depletion of heavy elements in the dust, such as through supernova events. The large sample of DGS galaxies surveyed from MIR to the submm, having widely varying metallicities, allows us to rigorously test various chemical evolution models (e.g. Edmunds 2001; Dwek 1998; Galliano et al. 2008). Tighter constraints were put on the metallicity dependence of the $\mathrm{D} / \mathrm{G}$ by Galametz et al. (2011) but the behavior below $Z=12+\log (\mathrm{O} / \mathrm{H})$ 
$\sim 8$ has been ambiguous due to lack of low metallicity observations. Investigating the behavior of $\mathrm{D} / \mathrm{G}$ with metallicity in the DGS will help to decipher the origin of the dust: for example, from supernovae versus evolved low mass stars.

One popular way to get at possible CO-dark molecular gas is via the dust mass. Using the dust mass determined from observations, along with an assumed D/G (most often used as a linear or near-linear relation with metallicity), can give the total gas mass which can be compared to that observed in HI and CO. This has been shown to reveal gas reservoirs not emitting in CO or HI (e.g. Leroy et al. 2011, and references within). Measuring dust mass and using an expected $\mathrm{D} / \mathrm{G}$ has also been used to determine the CO-to- $\mathrm{H}_{2}$ conversion factor for high- $z$ galaxies (Magdis et al. 2012; Magnelli et al. 2012). Such methods to get at any possible dark gas, however, require confidence in the dust mass determination as well as understanding metallicity variations and the $\mathrm{D} / \mathrm{G}$ variations throughout a galaxy.

Possible uncertainties in the total dust mass, as well as the total molecular gas reservoir, again highlights the difficulty in understanding the ISM of low metallicity galaxies and the local conditions for star formation. With the new Herschel data and self consistent modeling of the gas and dust together we can begin to unravel the mysteries of the ISM physics in dwarf galaxies.

\section{Source Selection}

Until very recently, we have had information only on a small number of relatively well-known dwarf galaxies with metallicities mostly ranging from $1 / 2$ to $1 / 10 Z_{\odot}$, with the exceptions of I Zw 18 and SBS 0335-052, which are 1/50 and 1/40 $Z_{\odot}$. Deep optical emission line and photometric observations from the Hamburg/SAO Survey and the First and Second Byurakan Surveys (e.g. Izotov et al. 1991; Ugryumov et al. 2003) have unveiled 
a treasure trove of low metallicity galaxies, with metallicity values ranging as low as $1 / 50$ $Z_{\odot}$, including a large number of ELM galaxies. We have chosen a broad sample of 50 galaxies ranging from very low metallicity $\left(\sim 1 / 50 Z_{\odot}\right)$ to moderate metallicity $\left(\sim 1 / 3 Z_{\odot}\right)$ with $\sim 1 / 3$ of the sample targeting ELMs. The first panel in Figure 3 shows the distribution of the metallicity values of our sample. Beyond the Local Group sources, our DGS sample was selected from several surveys including the Hamburg/SAO Survey and the First and Second Byurakan Surveys picking up emission line galaxies. Our target sources come from follow-up observations which specifically identified the metal-deficient Blue Compact Dwarf galaxies (BCDs) from the survey (e.g. Izotov et al. 1991; Ugryumov et al. 2003; Popescu \& Hopp 2000), as well as the sample of Izotov \& Thuan (1999) and Hopkins et al. (2002), which contain some of the better-studied BCDs. The sources range from the nearest neighbor galaxies, the Large and Small Magellanic Clouds (LMC and SMC; $50 \mathrm{kpc)}$ ) and NGC 6822 (500 kpc) to the more distant Haro 11 (92 Mpc) and HS0052+2536 (191 Mpc).

The design of the DGS is an attempt to obtain a statistically significant sample of about 9 galaxies in 7 bins over a wide metallicity range: $12+\log (\mathrm{O} / \mathrm{H})$ ranging from 8.4 $\left(1 / 3 Z_{\odot}\right)$, in bins of $12+\log (\mathrm{O} / \mathrm{H})=0.2$ (a factor of 1.4 difference) to provide an accuracy of $\sim 30 \%$. As can be seen from Figure 3 , it was not possible to have a uniform sample in terms of metallicity, due to lack of availability or detectability of such extreme low metallicity sources in the local Universe. We also did not insist on populating our highest metallicity bins, since metal-rich galaxies are plentiful in other surveys, such as the Herschel Reference Survey (Boselli et al. 2010; Ciesla et al. 2012), Herschel Virgo Cluster Survey (Davies et al. 2012) and the KINGFISH survey (Kennicutt et al. 2011), for example.

The target list is presented in Table 2 along with galaxy characteristics that include: Galaxy name (column 1), RA and DEC (columns 2 and 3) and distance (column 4). Any distance quoted here not referenced to the literature, has been determined using the 

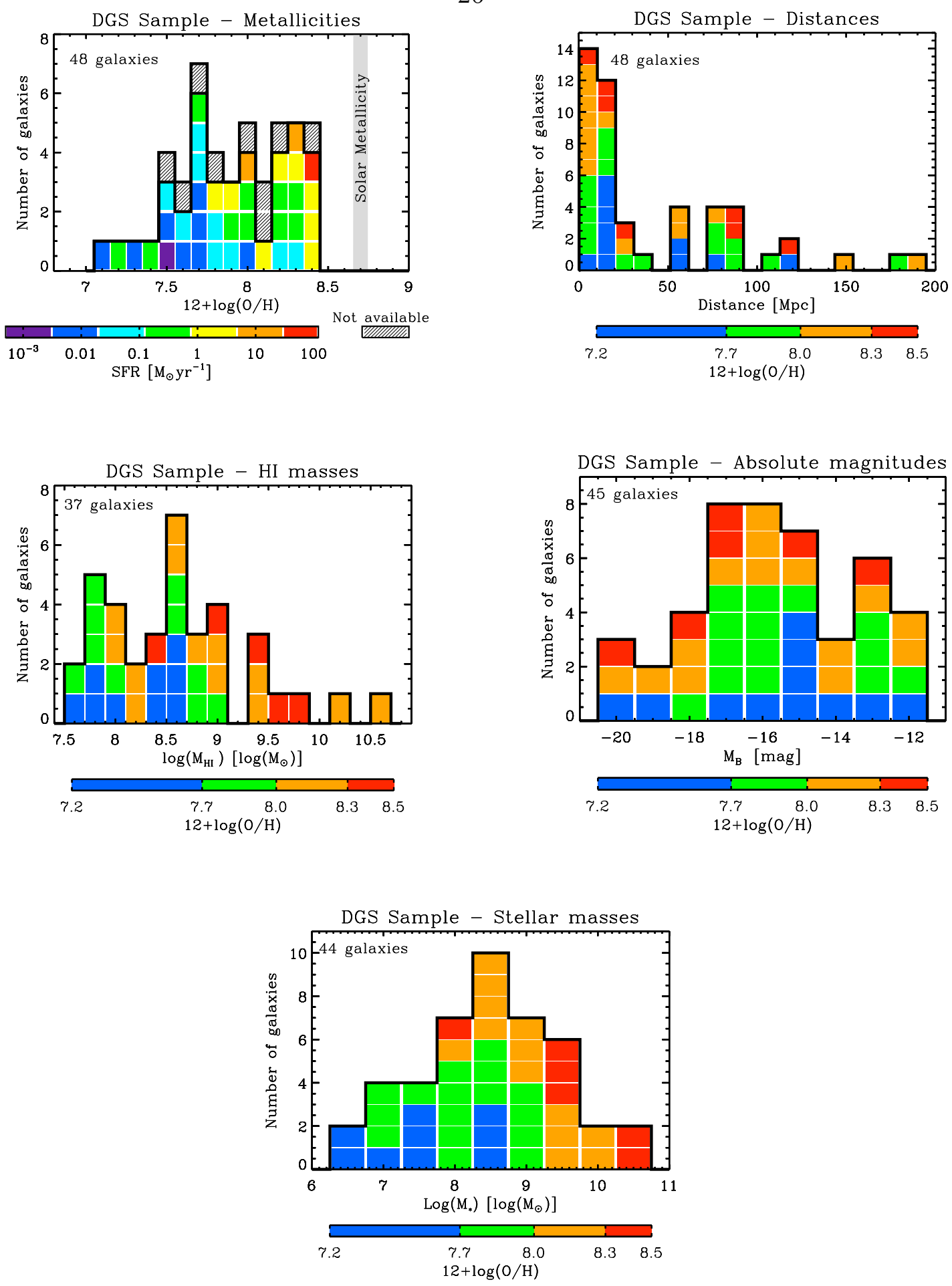

Fig. 3.- Properties of the DGS sample : Distribution of metallicity and SFR properties (top left) computed from $L_{T I R}$ as in Dale \& Helou (2002), if IRAS or Spitzer data is available. Otherwise SFR was determined from $\mathrm{H}_{\alpha}$ or $\mathrm{H}_{\beta}$ (Kennicutt 1998). When data were not available they are labeled 'not available'. Histograms of distances (top right), HI masses (middle left), absolute B magnitude (middle right) and stellar masses, $M_{*}$ (bottom), from the formula of Eskew et al. (2012) using 3.6 and $4.5 \mu \mathrm{m}$. The color scale represents the range of star formation rate for the top panel and metallicity for the others. 
velocities given in NED and the Mould et al. (2000) model, assuming $\mathrm{H}_{0}=70 \mathrm{~km} \mathrm{~s}^{-1}$ $\mathrm{Mpc}^{-1}$. Metallicities (in terms of oxygen abundances) are in column $5 ; \mathrm{M}_{B}$ (column 6 ), galaxy size in terms of $\mathrm{D}_{25}$ (column 7), $M_{*}$, stellar mass, from the formula of Eskew et al. (2012) using 3.6 and $4.5 \mu \mathrm{m}$ (column 8); $\mathrm{M}_{H I}$, mass of HI (column 9); $L_{T I R}$ determined from Spitzer bands (Dale \& Helou 2002) (column 10) and star formation rate (SFR) using the IR-based calibration of Kennicutt (1998) (column 11).

Determination of absolute metallicity values can vary, depending on the calibration method used (e.g. Kewley \& Ellison 2008, and references within). In order to ensure homogeneity in the calibration of the metallicity values in the DGS sample, metallicities were recomputed from optical line intensities following the methods of Izotov et al. (2006) (I06) and Pilyugin \& Thuan (2005) (PT05) and compared. The method of I06 uses the direct measurement of the electron temperature $\mathrm{T}_{e}[\mathrm{OIII}]$ from the [OIII] $\lambda 4363$ line. Pilyugin \& Thuan (2005) is an empirical calibration of the metallicity value using the $R_{23}$ ratio determined from strong emission lines: $\mathrm{R}_{23}=([\mathrm{OII}] \lambda 3727+[\mathrm{OIII}] \lambda \lambda 4959,5007) / \mathrm{H} \beta$. To break the degeneracy between the upper and lower branches of the calibration (Kobulnicky \& Kewley 2004), we use the $[\mathrm{NII}] \lambda 6584 /[\mathrm{OII}] \lambda 3727$ ratio as advised in Kewley \& Ellison (2008). The mean of the variations between the PT05 and I06 values for this sample is about 0.1 dex (see Appendix A for comparison and discussion). For the presentation of the DGS, we choose the PT05 values for consistency (Table 2). Uncertainties on the metallicity values are computed by propagating the errors in the observed lines.

The wide range in the distribution of the DGS sample in metallicity, SFR, distance, $\mathrm{M}_{H I}, \mathrm{M}_{B}$, and $\mathrm{M}_{*}$ are presented as histograms in Figure 3 . 


\section{Observation strategy and data reduction}

The DGS observed both the dust and gas components in the target sources with a total observing time of 230h. With Herschel the FIR PACS bands of 70, 100 and $160 \mu \mathrm{m}$ and the submm bands of SPIRE at 250, 350 and $500 \mu \mathrm{m}$ are mapped. The fine-structure lines of [CII] $158 \mu \mathrm{m},[\mathrm{OIII}] 88 \mu \mathrm{m},[\mathrm{OI}] 63,[\mathrm{OI}] 145 \mu \mathrm{m}$ and in few cases [NIII] $57 \mu \mathrm{m},[\mathrm{NII}]$ $122 \mu \mathrm{m}$ and [NII] $205 \mu \mathrm{m}$ have been observed. SPIRE FTS submm observations have been obtained for 4 DGS sources.

The survey contains 37 compact galaxies with sizes ranging from 0.08 ' to 1.2 ' and 11 well-studied extended galaxies (Table 2), with the most extended ones being NGC 625, NGC 2366, NGC 4214, NGC 4449, NGC 4861 and the Local Group galaxies, IC 10, NGC 6822 and the Magellanic Clouds. All of these galaxies have also been observed with Spitzer photometry bands and the Spitzer IRS (Figure 4).

Since the low surface brightness dust in diffuse metal-poor regions is important in studying the dust processing and may be associated with the extended HI structures in dwarf galaxies, we planned observations to completely cover the star forming regions and

where possible much of the HI structures. A nominal size of at least $2 \times \mathrm{D}_{25}$ was considered for most photometry maps.

\subsection{PACS imaging}

As 2 of the 3 PACS bands (Poglitsch et al. 2010) can be observed simultaneously (either $70 \mu \mathrm{m}$ and $160 \mu \mathrm{m}$ or $100 \mu \mathrm{m}$ and $160 \mu \mathrm{m}$ ), 2 sets of observations were required to obtain all of the 3 PACS bands. Mapping the largest galaxies of the Local Group with the 3 PACS bands required $\sim 10$ h of observing time per galaxy. For each band, we obtained two sets of maps with scanning angles separated by 90 degrees to reduce the possibility of 


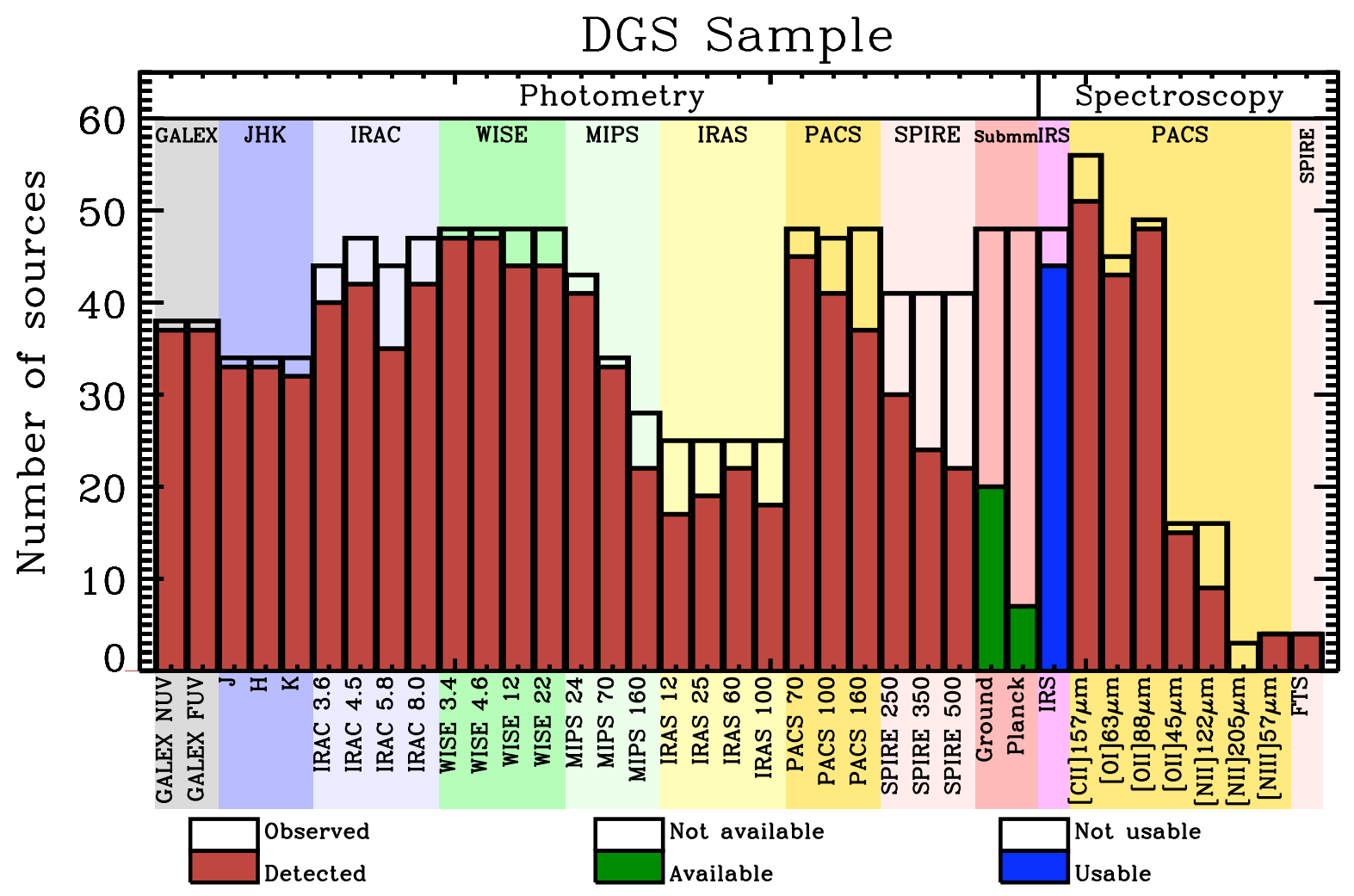

Fig. 4.- Histogram of the number of observed and detected Herschel sources in the DGS program along with the accompanying ancillary data that has been collected. Note that PACS spectroscopy includes 7 individual LMC and SMC sources, thus accounting for the larger number (55 sources) in the histogram while the accompanying LMC and SMC Herschel photometry data is in the HERITAGE program (Meixner et al. 2013, submitted). 
striping in the maps. All of the sources were observed in the PACS scan-map mode with map sizes varying from 4'x4' to 30'x30' using medium scan speed.

The PACS data were reduced using the Herschel Interactive Processing Environment (HIPE) version 7.0. The details of the data reduction and the various map reconstruction techniques and tests are described in Rémy-Ruyer et al. 2013a (in preparation).

Three different map-making methods, PhotProject, MADmap, and Scanamorphos (Roussel et al. 2012, submitted), were performed on all of the DGS PACS data and resulting aperture photometry values were compared to determine the preferred method. The PACS bolometer thermal drifts, in the form of $1 / \mathrm{f}$ noise, leave imprints on the maps which manifest in large scale spatial structures. These are removed in PhotProject via high-pass filtering, having the tendancy to suppress extended, large scale emission. The MADmap method creates maximum likelihood maps from the time ordered data, assuming the the detector noise is Gaussian, which is not the case for the $1 / \mathrm{f}$ noise. Extended features tend to be reproduced somewhat better than in PhotProject maps. Scanamorphos takes advantage of the redundancy in the maps and accounts for the correlated and uncorrelated, non-thermal noise. From Level 1 data treatment in HIPE, the data were exported into Scanamorphos and maps reconstructed using the default options. After detailed tests comparing all methods (Rémy-Ruyer et al. 2013a in preparation), PhotProject was in most cases found to be more adapted for point sources while Scanamorphos was chosen for the extended galaxies.

The FWHM beam sizes for PACS bands are 5.2", 7.7" and 12" for the 70, 100 and 160 $\mu \mathrm{m}$ observations while final pixel sizes are 2", 2" and 4", respectively. The sensitivities we achieve in the PACS observations are normally a $1 \sigma$ value ranging $\sim 1.2-8.4 \mathrm{MJy} \mathrm{sr}^{-1}$ for 70 and $100 \mu \mathrm{m}$ and 0.6 - 5.6 $\mathrm{MJy} \mathrm{sr}^{-1}$ for $160 \mu \mathrm{m}$ (Table 3). Calibration uncertainties are 3, 3, and $5 \%$ for the 70, 100 and $160 \mu \mathrm{m}$ PACS bands. We obtain a S/N of at least 10 for 
the brightest galaxies or the brightest regions in galaxies, and 3 to $5 \sigma$ detections typically for the fainter (lowest metallicity) galaxies. 


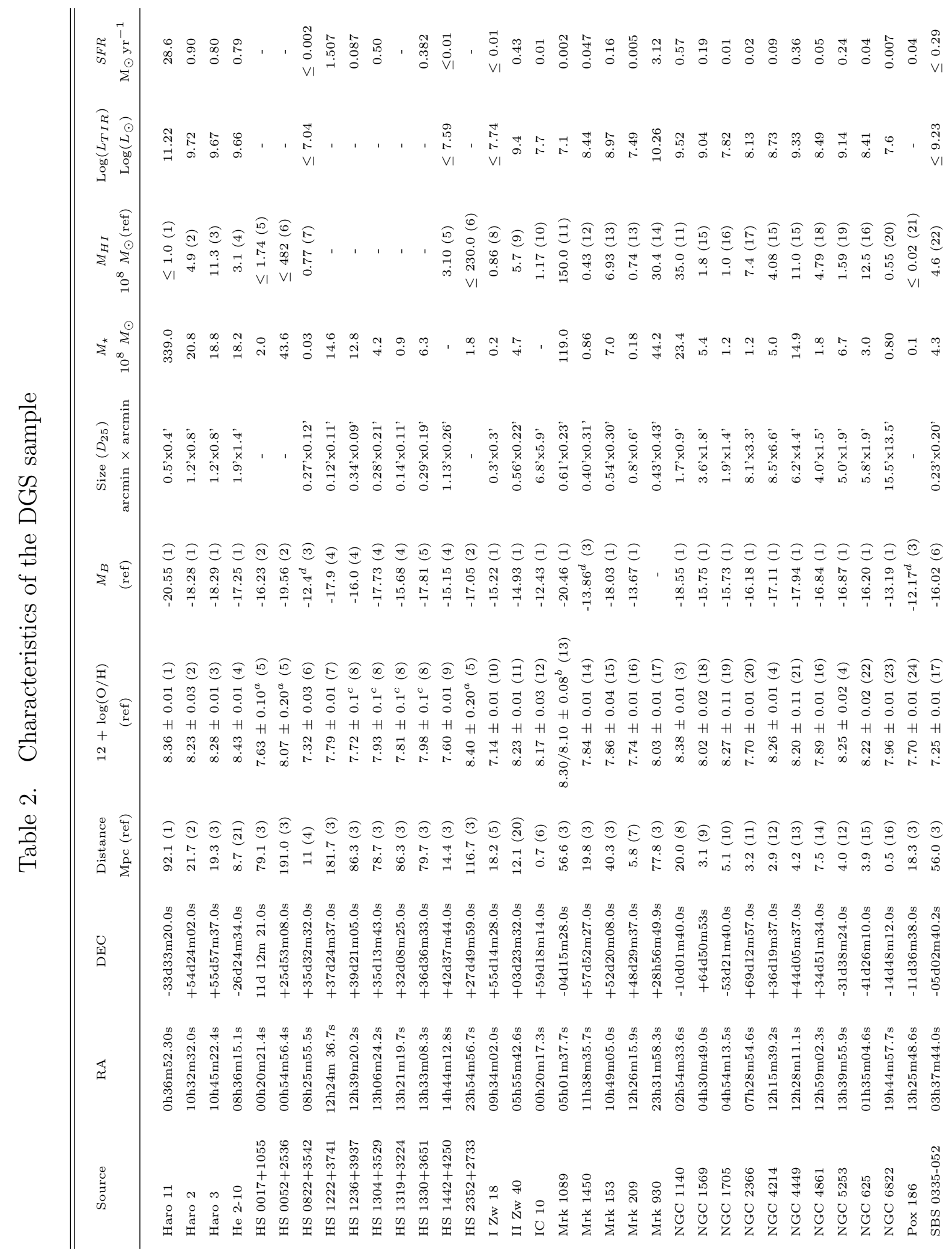




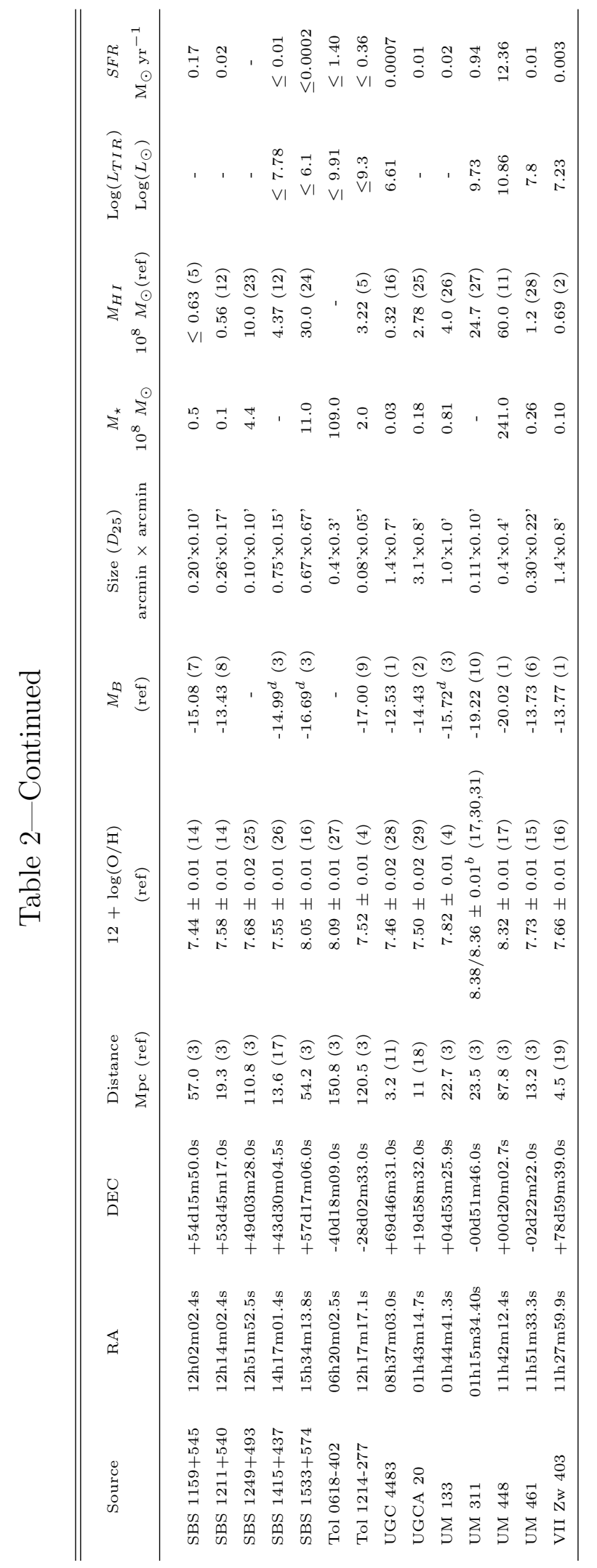




\section{Notes to Table 2}

Positions are from the NASA/IPAC Extragalactic Database (NED). Distances are from the literature. Metallicity values in $12+$ log $(\mathrm{O} / \mathrm{H})$ are determined as in Pilyugin \& Thuan 2005 (PT05) using the $\mathrm{R}_{23}$ ratio except for where noted. See text for further details on methods. Absolute $\mathrm{B}$ magnitude, $\mathrm{M}_{B}$, is derived from the corresponding apparent magnitudes from NED. $\mathrm{D}_{25}$ is the galaxy diameter from NED. $\mathrm{M}_{\star}$ is stellar mass converted from 3.6 and $4.5 \mu \mathrm{m}$ following the method of Eskew et al. (2012). $\mathrm{M}_{H I}$ is from the literature. Total infrared luminosities ( $L_{T I R}$ ) have been computed using the formula of Dale \& Helou (2002) and the Spitzer fluxes from Bendo et al. (2012a) when available. Star formation rates (SFR) has been derived from $L_{T I R}$ using the formula of Kennicutt (1998) or from $\mathrm{H} \alpha$ and $\mathrm{H} \beta$ when no IR data is available. A dash indicates that no data is available.

References for distances : (1) Bergvall et al. 2006) ; (2) Kennicutt et al. 2003) ; (3) this work, calculated from the redshifts available in NED, the Hubble flow model from Mould et al. 2000 and assuming $\mathrm{H}_{0}=70 \mathrm{~km} \mathrm{~s}^{-1} \mathrm{Mpc}^{-1}$; (4) Pustilnik et al. 2003 ; (5) Aloisi et al. 2007) ; (6) Kim et al. 2009) ; (7) Schulte-Ladbeck et al. 2001) ; (8) Moll et al. 2007) ; (9) Grocholski et al. 2012 ; (10) Tosi et al. (2001 ; (11) Karachentsev et al. 2002 ; (12) Karachentsev et al. 2004) ; (13) Karachentsev et al. 2003 ; (14) de Vaucouleurs et al. 1991) ; (15) Cannon et al. 2003) ; (16) Gieren et al. 2006 ; (17) Aloisi et al. 2005) ; (18) Sharina et al. 1996 ; (19) Lynds et al. 1998 ; (20) Bordalo et al. 2009) ; (21) Tully 1988

References for metallicities : (1) Guseva et al. 2012; (2) Kong \& Cheng 2002 ; (3) Izotov et al. 2004 ; (4) Kobulnicky et al. (1999) ; (5) Ugryumov et al. 2003) ; (6) Pustilnik et al. 2003) ; (7) Izotov et al. 2007) ; (8) Popescu \& Hopp 2000 ; (9) Guseva et al. 2003a ; (10) Izotov \& Thuan (1999); (11) Guseva et al. 2000); (12) Magrini \& Gonçalves (2009); (13) López-Sánchez \& Esteban 2010) ; (14) Izotov et al. (1994) ; (15) Izotov et al. 2006 ; (16) Izotov et al. 1997) ; (17) Izotov \& Thuan (1998) ; (18) Kobulnicky \& Skillman (1997) ; (19) Lee \& Skillman 2004 ; (20) Saviane et al. 2008) ; (21) McCall et al. 1985 ; (22) Skillman et al. 2003 ; (23) Lee et al. 2006 ; (24) Guseva et al. 2007) ; (25) Thuan et al. 1995; (26) Guseva et al. 2003b) ; (27) Masegosa et al. 1994 ; (28) van Zee \& Haynes 2006 ; (29) van Zee et al. (1996) ; (30) Moles et al. 1994; (31) Pilyugin \& Thuan 2007)

${ }^{a}$ For these galaxies, no line intensities were available in the literature to determine the metallicities using the PT05 method. We use Ugryumov et al. 2003) where the [OIII] $\lambda 4363$ line is used to obtain the electron temperature and the method from Izotov et al. (1994). I06 is an updated version of the method of Izotov et al. 1994.

${ }^{b}$ These galaxies are part of a group of galaxies. We list the value of the metallicity for the galaxy only ( $1^{s t}$ value quoted) and for the whole group ( $2^{\text {nd }}$ value quoted), which is the mean of all of the metallicities in the group. For Mrk1089, the galaxy is region A-C from López-Sánchez \& Esteban 2010. For UM311, the galaxy is region 3 following Moles et al. 1994.

${ }^{c}$ For these galaxies, no line intensity uncertainties, from which the metallicities are determined, are quoted. We assume a conservative value of 0.1 which is the mean of the variation of the difference between this metallicity calibration, PT05, and the I06 method (see Appendix A).

References for apparent magnitudes : (1) de Vaucouleurs et al. (1991) ; (2) Ugryumov et al. 2003) ; (3) Gil de Paz \& Madore 2005) (4) Vennik et al. (2000) ; (5) Ugryumov et al. 2001) ; (6) Maddox et al. (1990) ; (7) SDSS DR4 ; (8) Paturel et al. 2003) ; (9) Fricke et al. (2001) ; (10) Smoker et al. (2000)

$d$ corrected for Galactic extinction

References for HI masses : (1) Bergvall \& Östlin 2002 ; (2) Thuan et al. 2004) ; (3) Gordon \& Gottesman 1981) ; (4) Sauvage et al. 1997) ; (5) Pustilnik \& Martin 2007) ; (6) MacHattie et al in preparation ; (7) Chengalur et al. 2006 ; (8) van Zee et al. (1998; (9) Bettoni et al. 2003 ; (10) Mateo 1998) ; (11) Davoust \& Contini 2004) ; (12) Huchtmeier et al. 2005 ; (13) Thuan \& Martin (1981) ; (14) Hopkins et al. 2002) ; (15) Walter et al. 2008) ; (16) Karachentsev et al. 1999) ; (17) Hunter et al. 2011) ; (18) van Eymeren et al. (2009) ; (19) López-Sánchez et al. 2012); (20) Koribalski et al. 2004); (21) Begum \& Chengalur 2005) ; (22) Ekta et al. 2009) ; (23) Pustilnik et al. 2002) ; (24) Thuan et al. 1999) ; (25) derived from Martin 1998) ; (26) Ekta \& Chengalur 2010 ; (27) Smoker et al. 2000) ; (28) Engelbracht et al. 2008 and references within. 
The improvement in spatial resolution of Herschel over Spitzer can be immediately

seen in Figure 5, presenting the nearby (5 Mpc) galaxy, NGC 1705, in the DGS (O'Halloran et al. 2010). Two clusters that are blended at FIR wavelengths with Spitzer are resolved with PACS on Herschel, allowing for detailed studies of the cooler dust properties for the first time.

\subsection{PACS spectroscopic observations}

The PACS spectrometer footprint consists of $5 \times 5$ pixels of $9.4 "$ each, with a total field of view of $47 " \times 47$ ". Each spatial pixel covers 16 spectral elements. In only a few cases were all of the brightest 7 FIR fine structure lines observed (Table 4): $158 \mu \mathrm{m}$ [CII], 63 and $145 \mu \mathrm{m}$ [OI], $88 \mu \mathrm{m}$ [OIII], $57 \mu \mathrm{m}$ [NIII] and the 122 and $205 \mu \mathrm{m}$ [NII] lines. In all but a few of the faintest galaxies, at least the [CII] and [OIII] were observed (Figure 4). The spectral resolutions for the [CII], [OIII] and [OI] lines are: $\sim 1250\left(240 \mathrm{~km} \mathrm{~s}^{-1}\right)$, $\sim 2400\left(125 \mathrm{~km} \mathrm{~s}^{-1}\right)$, and $\sim 3300\left(90 \mathrm{~km} \mathrm{~s}^{-1}\right)$, respectively. The chop/nod line observing mode was used for the compact sources which fit within the spectrometer footprint. For sources too extended for chopping (maximum chop of $+/-3$ arcmin), we made raster maps using the unchopped grating scan mode, which replaced the decomissioned wavelength switching mode, used only for LMC/N11B (Lebouteiller et al. 2012). The data have been reduced to Level 1 within HIPE and then input into PACSman, an IDL package to handle line-fitting and map projection for PACS observations (Lebouteiller et al. 2012). Maps are projected on a subpixel grid with with each final pixel size about $3 " \times 3 "$, roughly $1 / 3$ of the original spatial pixel. Details of the PACS data processing of the survey will be presented in Cormier et al. (2013, in preparation).

The size of the spectroscopic maps vary depending on source and line. (Table 4). With the FWHM of $9.5 "$ at $60-90 \mu \mathrm{m}$ and $11.5 "$ at $150 \mu \mathrm{m}$, the spatial resolution of Herschel 


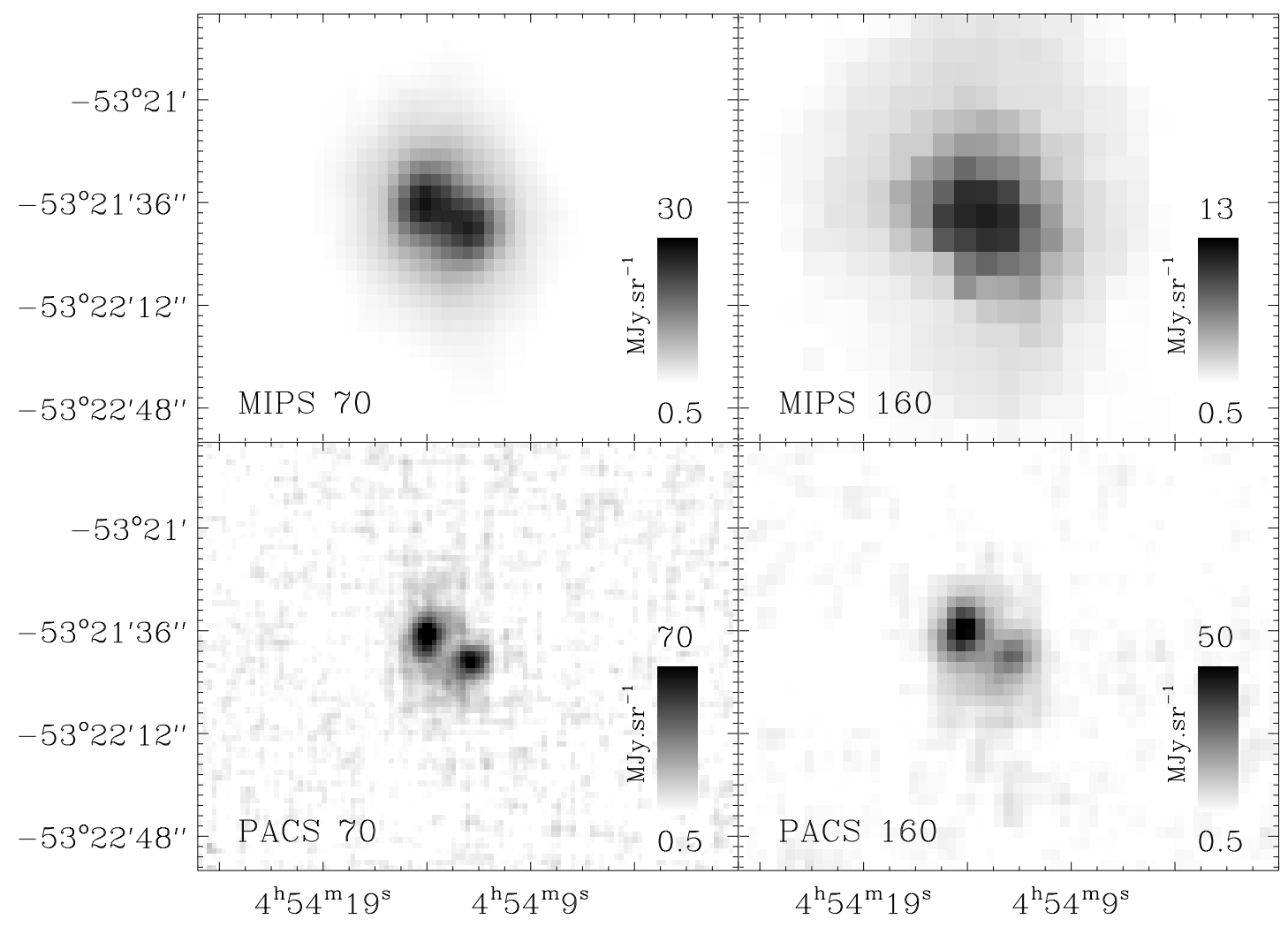

Fig. 5.- Improvement in spatial resolution of Herschel in the FIR over Spitzer is demonstrated on the DGS galaxy NGC1705 (O'Halloran et al. 2010) in the comparison of MIPS (top row) with PACS (bottom row). The two clusters are well separated in both PACS images whereas they are blurred in MIPS images and indistinguishable at $160 \mu \mathrm{m}$. The FWHM of the PACS beams for the 70 and $160 \mu \mathrm{m}$ bands are 5.7" and 12" while for the MIPS bands the FWHM values are 18" and 38". 
allows us to explore the morphology of the galaxies, and determine the physical properties traced by the FIR cooling lines for the first time within dwarf galaxies, not just around the brightest star forming regions. We achieved a dynamic range in flux values greater than or equal to 10 in [CII], [OI] $63 \mu \mathrm{m}$, and [OIII] observations - in some cases as high as 100, in the brightest sources, thus, providing the sensitivity to extract physical information from the more diffuse ISM. In some Local Group galaxies, the S/N can be as high as 100 for some lines in the extended regions. For the more compact sources (often more distant), the median S/N is around: 13 for [CII], 18 for [OI] $63 \mu \mathrm{m}, 38$ for [OIII], 6 for [NII]122 $\mu \mathrm{m}$, and 15 for $[\mathrm{OI}] 145 \mu \mathrm{m}$. As an example of the kind of sensitivity brought to these studies by PACS, Madden et al. (1997) detected low surface brightness extended [CII] emission in IC 10 at the level of $1 \times 10^{-15} \mathrm{~W} \mathrm{~m}^{-2}$ in a $55 "$ beam of FIFI on the KAO. If such emission were spread uniformly over the beam, the expected signal at the PACS spatial resolution would be $\sim 4 \times 10^{-17} \mathrm{~W} \mathrm{~m}^{-2}$. In comparison, the sensitivity of PACS after single spectroscopic integration (effective on-source time of 150s) for this line, in an equivalent KAO beam, is $2.9 \times 10^{-18} \mathrm{~W} \mathrm{~m}^{-2}$, and thus was rapidly detected with PACS with a $\mathrm{S} / \mathrm{N}$ ranging from 10 to 100 across IC10.

\subsection{SPIRE imaging}

SPIRE (Griffin et al. 2010) observed 250, 350 and $500 \mu \mathrm{m}$ bands simultaneously, with FWHM beam sizes of 18.1", 25.2" and 36.6", respectively. The details of the data reduction are described in Rémy-Ruyer et al. 2013a (in preparation). Sixteen galaxies including those with sizes larger than 1.5' were observed in the large scan-map mode, while the remaining galaxies were observed in small scan-map mode. Observations were performed as cross-scans, thus removing $1 / \mathrm{f}$ noise and at nominal speed $\left(30 " \mathrm{~s}^{-1}\right)$. SPIRE data were processed mainly through HIPE, using the waveletDeglitcher. The residual baseline 
subtraction applies the median of the bolometer timelines for the whole observation, not only a single scan leg (BriGadE method, Smith et al, in preparation). Calibration uncertainties are estimated to be $\sim 7 \%$ for all SPIRE bands. At $250 \mu \mathrm{m}, 8$ sources are detected and unresolved and 11 are not detected. At $500 \mu \mathrm{m}, 5$ sources are unresolved and 19 are not detected (Figure 3). The $1 \sigma$ sensitivities we achieved with SPIRE range from

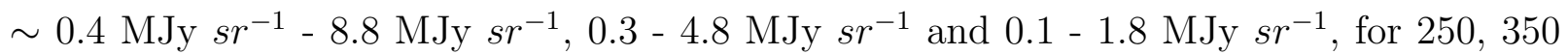
and $500 \mu \mathrm{m}$, respectively (Table 3) and final pixel sizes are 6", 8" and 12", respectively. Mapping the 11 larger galaxies takes about $1 \mathrm{~h}$ at most per galaxy to reach the desired sensitivities with SPIRE while the small scan mode required about 10 minutes per galaxy. 
Table 3. Herschel PACS and SPIRE $1 \sigma$ rms values (MJy sr ${ }^{-1}$ ) of final maps.

\begin{tabular}{|c|c|c|c|c|c|c|}
\hline Source & $70 \mu \mathrm{m}$ & $100 \mu \mathrm{m}$ & $160 \mu \mathrm{m}$ & $250 \mu \mathrm{m}$ & $350 \mu \mathrm{m}$ & $500 \mu \mathrm{m}$ \\
\hline Haro11 & 5.29 & 7.18 & 2.55 & 0.87 & 0.52 & 0.18 \\
\hline Haro2 & 4.95 & 4.87 & 2.06 & 0.87 & 0.51 & 0.20 \\
\hline Haro3 & 7.60 & 7.54 & 5.57 & 1.01 & 0.68 & 0.25 \\
\hline $\mathrm{He} 2-10$ & 8.46 & 8.27 & 4.10 & 1.04 & 0.62 & 0.30 \\
\hline HS0017+1055 & 2.93 & 2.96 & 1.27 & 0.62 & 0.33 & 0.12 \\
\hline HSO052+2536 & 2.89 & 2.72 & 1.39 & 0.76 & 0.36 & 0.15 \\
\hline HS0822+3542 & 2.33 & 2.01 & 1.04 & - & - & - \\
\hline HS1222+3741 & 2.90 & 3.07 & 1.36 & - & - & - \\
\hline HS1236+3937 & 3.24 & 3.28 & 1.46 & 0.77 & 0.44 & 0.21 \\
\hline HS1304+3529 & 3.24 & 4.08 & 1.55 & 0.94 & 0.49 & 0.16 \\
\hline HS1319+3224 & 1.91 & 1.90 & 1.03 & - & - & - \\
\hline HS1330+3651 & 2.38 & 2.40 & 0.98 & - & - & - \\
\hline $\mathrm{HS} 1442+4250$ & 3.83 & 3.54 & 1.78 & 0.93 & 0.56 & 0.21 \\
\hline HS2352+2733 & 2.30 & 2.11 & 0.88 & 0.72 & 0.47 & 0.13 \\
\hline IZw18 & 1.94 & 1.80 & 0.98 & 0.48 & 0.24 & 0.10 \\
\hline IC10 & 4.45 & 4.71 & 3.55 & 8.82 & 4.79 & 1.82 \\
\hline IIZw40 & 5.23 & 5.09 & 3.15 & 1.67 & 0.81 & 0.36 \\
\hline Mrk1089 & 7.88 & 7.77 & 6.02 & 1.14 & 0.64 & 0.29 \\
\hline Mrk1450 & 2.67 & 2.67 & 1.22 & 0.88 & 0.77 & 0.37 \\
\hline Mrk153 & 4.42 & 4.72 & 2.52 & 0.56 & 0.41 & 0.20 \\
\hline Mrk209 & 7.08 & 7.28 & 3.79 & 0.62 & 0.38 & 0.13 \\
\hline Mrk930 & 3.78 & 3.49 & 1.73 & 0.79 & 0.40 & 0.15 \\
\hline NGC1140 & 3.77 & 3.68 & 1.77 & 0.87 & 0.50 & 0.19 \\
\hline NGC1569 & 3.78 & 3.80 & 2.07 & 2.38 & 1.28 & 0.46 \\
\hline NGC1705 & 4.08 & 3.96 & 1.79 & 0.86 & 0.54 & 0.25 \\
\hline NGC2366 & 3.28 & 3.27 & 1.44 & 0.65 & 0.39 & 0.17 \\
\hline NGC4214 & 3.79 & 3.66 & 1.72 & 0.91 & 0.56 & 0.25 \\
\hline NGC4449 & 3.45 & 3.34 & 1.63 & 0.83 & 0.48 & 0.21 \\
\hline NGC4861 & 3.20 & 3.06 & 1.40 & 0.80 & 0.49 & 0.22 \\
\hline NGC5253 & 3.43 & 3.35 & 1.57 & 0.90 & 0.49 & 0.21 \\
\hline NGC625 & 3.70 & 3.55 & 1.60 & 0.80 & 0.46 & 0.19 \\
\hline NGC6822 & 4.21 & 4.05 & 1.99 & 3.06 & 1.71 & 0.64 \\
\hline
\end{tabular}


Table 3-Continued

\begin{tabular}{lcccccc}
\hline \multicolumn{1}{c}{ Source } & $70 \mu \mathrm{m}$ & $100 \mu \mathrm{m}$ & $160 \mu \mathrm{m}$ & $250 \mu \mathrm{m}$ & $350 \mu \mathrm{m}$ & $500 \mu \mathrm{m}$ \\
\hline Pox186 & 3.69 & 3.34 & 1.40 & 0.67 & 0.39 & 0.18 \\
SBS0335-052 & 2.26 & 1.99 & 1.01 & 0.67 & 0.38 & 0.16 \\
SBS1159+545 & 2.65 & 1.96 & 0.98 & 0.58 & 0.33 & 0.16 \\
SBS1211+540 & 1.21 & 1.29 & 0.64 & 0.59 & 0.34 & 0.13 \\
SBS1249+493 & 3.45 & 3.34 & 1.45 & 0.80 & 0.49 & 0.17 \\
SBS1415+437 & 3.75 & 3.80 & 1.67 & - & - & - \\
SBS1533+574 & 5.35 & 5.27 & 2.29 & 1.18 & 0.87 & 0.39 \\
Tol0618-402 & 1.50 & 1.50 & 0.90 & - & - & - \\
Tol1214-277 & 1.53 & 1.44 & 0.73 & 0.64 & 0.38 & 0.18 \\
UGC4483 & 5.32 & 6.50 & 2.26 & 0.60 & 0.34 & 0.15 \\
UGCA20 & 3.65 & 3.80 & 1.55 & - & - & - \\
UM133 & 3.74 & 3.64 & 1.86 & 0.75 & 0.44 & 0.18 \\
UM311 & 3.63 & 3.46 & 1.55 & 0.82 & 0.51 & 0.21 \\
UM448 & 7.74 & NaN & 4.47 & 0.74 & 0.47 & 0.23 \\
UM461 & 3.81 & 3.67 & 1.79 & 0.79 & 0.56 & 0.20 \\
VIIZw403 & 7.22 & 7.46 & 3.48 & 0.55 & 0.31 & 0.15 \\
\hline
\end{tabular}

Note. - A dash indicates lack of data for this band. 


\subsection{SPIRE FTS observations}

The SPIRE Fourier Transform Spectrometer (FTS) covers 194 to $671 \mu \mathrm{m}$ (447 to $1550 \mathrm{GHz}$ ), providing simultaneous coverage, for example, of CO rotational lines ranging from $\mathrm{J}=4-3$ through $\mathrm{J}=13-12$, as well as other molecules and atomic fines structure lines, such as [NII] $205 \mu \mathrm{m}$ and [CI] lines at 492 and $809 \mathrm{GHz}$. The spectrometer array can be operated in three spectral resolutions, low $(\delta \nu=25 \mathrm{GHz})$, medium $(\delta \nu=7 \mathrm{GHz})$, and, high $(\delta \nu=1.2 \mathrm{GHz})$, and is split into the Spectrometer Long Wave array (SLW: 303 -671 $\mu \mathrm{m}$ ) and the Spectrometer Short Wave array (SSW: 194-313 $\mu \mathrm{m}$ ). The SLW and SSW consist of 7 and 17 unvignetted bolometers arranged in a hexagon pattern covering a field of view of about $3^{\prime} \times 3$ '. The beam ranges from 17 " to 40 " across the FTS wavelength range. Depending on the image sampling mode, the bolometer arrays are moved around the requested pointing within the FOV in 1-point (sparse), 4-point (intermediate), or, 16-point (full) jiggles, resulting in a map with beam spacings of 50.5", 25.3", or, 12.7", for the SLW, and, 32.5", 16.3", or, 8.1", for the SSW.

NGC 4214 and IC10 are observed with the FTS in high spectral resolution, using the sparse sampling mode for a total of 2.7 and 3.7h, respectively. He2-10 and 30 Doradus, the most massive star forming region in our neighboring LMC, are observed in intermediate sampling mode for 4.6 and $2.8 \mathrm{~h}$, respectively, and also with high spectral resolution. The SPIRE FTS data is first processed using the extended source calibration of the Herschel Science Center pipeline which assumes the source is uniformly distributed within the FOV. Since this assumption is not necessarily accurate, we correct for the source distribution using the photometry maps using the method introduced in $\mathrm{Wu}$ et al. (2013, in preparation). Calibration uncertainties in the SPIRE FTS range from about 5\% at the lower wavelength to $\sim 10 \%$ toward the long wavelength end. 


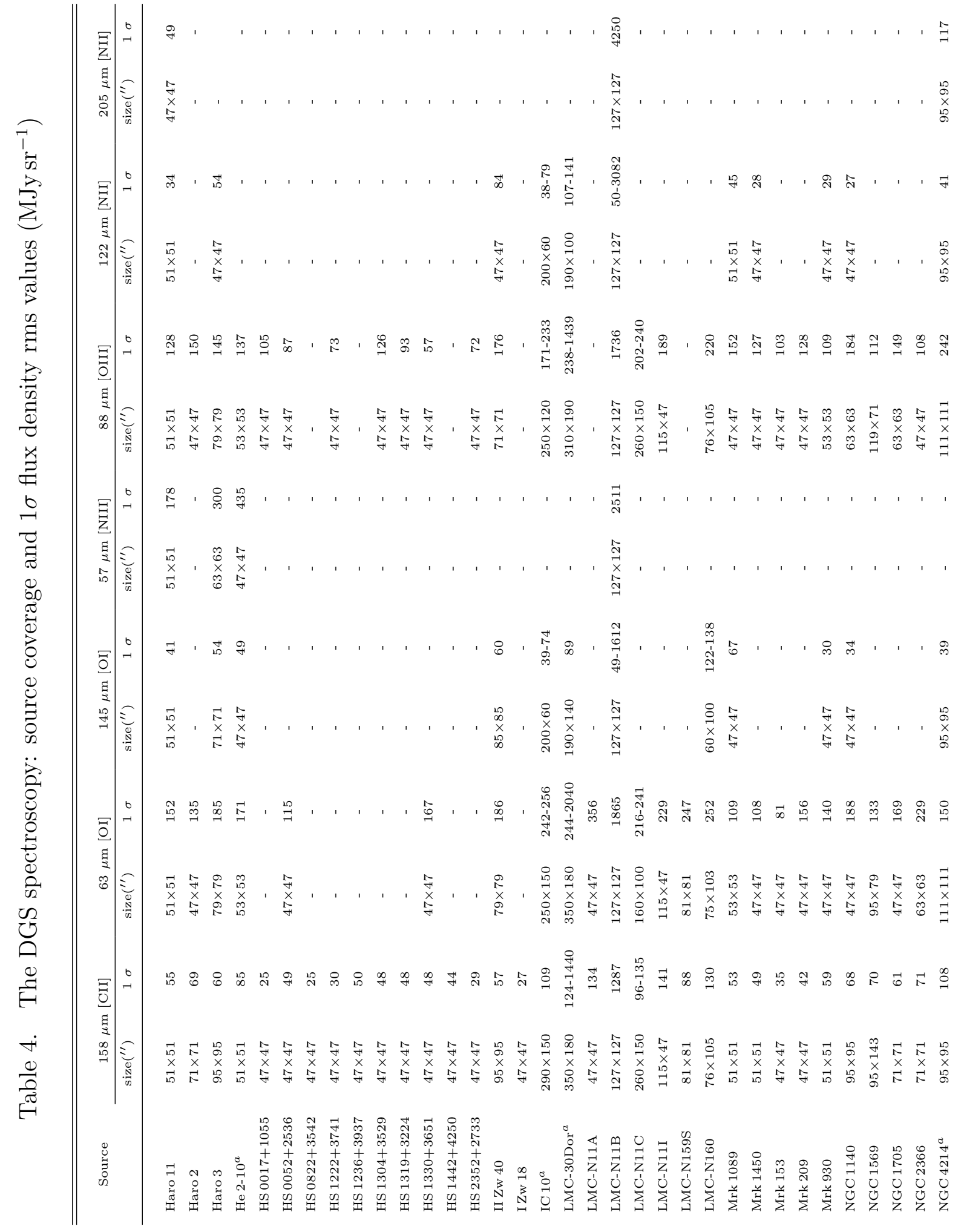




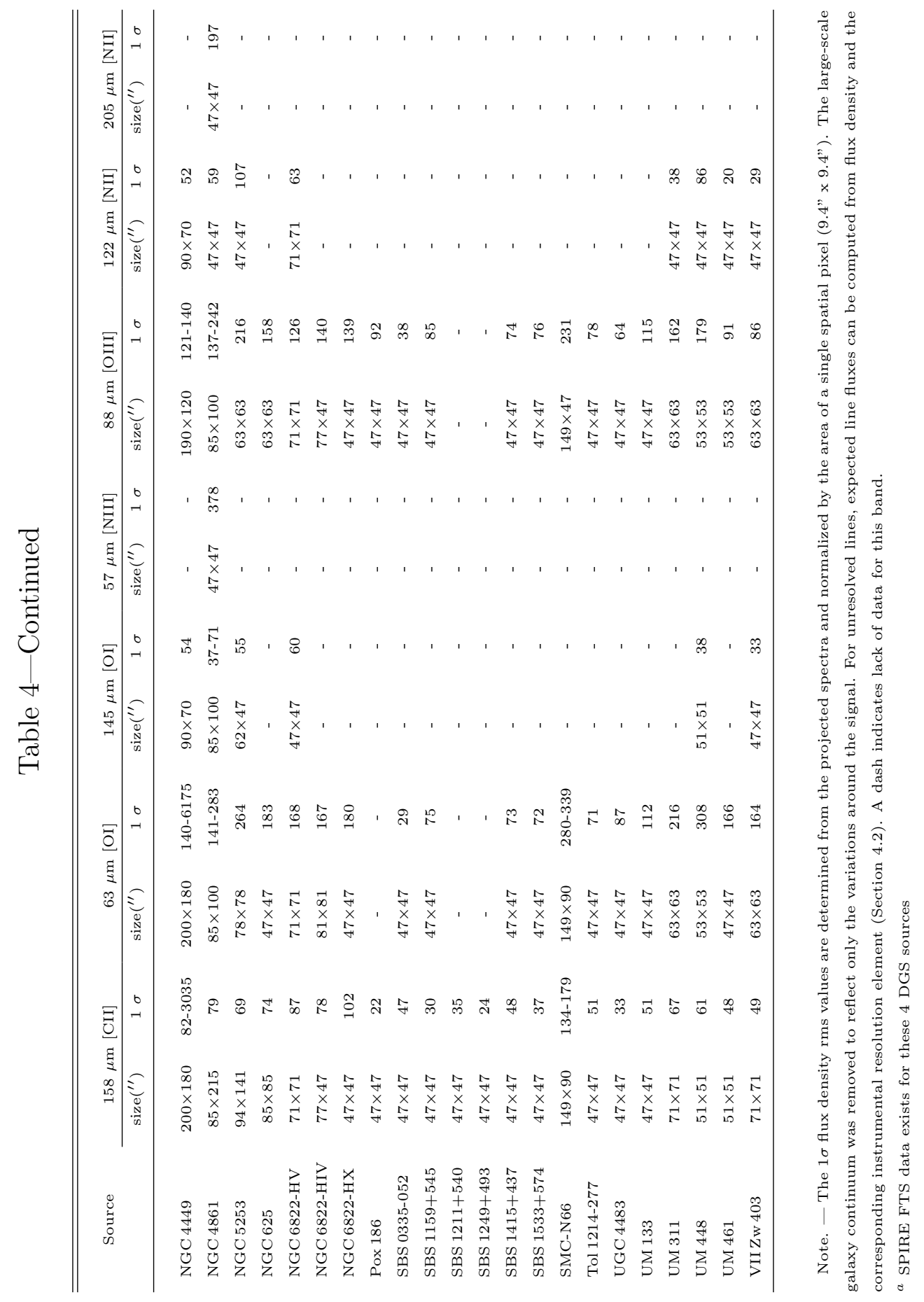




\subsection{Ancillary data - for a multiphase, multiwavelength approach}

The DGS galaxies have been observed with numerous ground-based and space-based observatories (Figure 4). Having the full range of UV to radio observations available opens

the door to some very powerful multiphase modeling of the dust and gas, and to relate this to a coherent picture of chemical evolution of galaxies. An important goal of the studies to come out of the DGS is to characterize the ISM as a function of metallicity and make the link to the ISM and star formation processes in truly primordial galaxies. With the vast multi-wavelength Herschel data set, along with the complementary data that exists or is being collected, the physical characteristics of the nebular gas, the photodissociation regions, the neutral gas and stellar properties can be characterized.

The first step in exploiting the Herschel observations has been the construction of the detailed database of existing observations from UV to radio wavelengths. In addition to the new PACS and SPIRE photometry and spectroscopy, the database includes the NIR to mm bands necessary to best characterize the dust properties using existing IRAS data, Spitzer MIR to FIR observations, as well as the critical ground-based submm observations: $850 \mu \mathrm{m}$, $870 \mu \mathrm{m}$ and $1.2 \mathrm{~mm}$ data from SCUBA/JCMT or SCUBA-2/JCMT, LABOCA/APEX, MAMBO/IRAM and, complementing the longer submm to radio wavelengths, Planck data. Information on the atomic, ionized and molecular gas phases includes the Spitzer IRS spectroscopy and is being complemented by data from the literature. A campaign of follow up observations to complete the molecular, atomic and dust submm/mm database is currently underway (Cormier et al. 2013, in preparation; Rémy-Ruyer et al. 2013b, in preparation). To characterize the stellar activity, 2MASS and GALEX data have been collected for the database.

The ancillary data compiled for the DGS sample to date include the following: 
- GALEX maps in FUV and NUV (1528 and 2271A) observations are collected from the STScI MAST archive (http://galex.stsci.edu/GR6/). These data give us a view into the star formation histories of the galaxies by capturing the direct UV emission from young stars to the most recent star formation activity (e.g. Hunter et al. 2010). The dust absorption properties in the UV provide additional constraints on the SED modeling of the dust emission properties in the MIR to FIR. GALEX observations exist for all but 9 of the DGS sample.

- 2MASS observations have been compiled from IRSA (http://irsa.ipac.caltech.edu/) and exist for 34 galaxies in the DGS. The J, H and $\mathrm{K}$ bands from 2MASS provide direct access to the emission from the oldest stellar population.

- Spitzer has observed all 50 targets of the DGS with various combinations of IRAC bands at 3.6, 4.5, 5.8 and $8.0 \mu \mathrm{m}$ (beam sizes : 1.7", 1.7", 1.9", 2.0") and MIPS bands at 24, 70 and $160 \mu \mathrm{m}$ (beam sizes : 6", 18", 38"). The IRAC and MIPS micron data consist of a combination of archival data and the cycle 5 program: Dust Evolution in Low-Metallicity Environments (P.I. F. Galliano; ID: 50550). The MIPS data are reduced and compiled in Bendo et al. (2012b) and recently publicly released. Spitzer IRAC bands cover the emission from the MIR-emitting small grains and the PAHs, the most important species in galaxies for gas heating via the photoelectric effect (Bakes \& Tielens 1994). The MIR and FIR range of the dust SEDs and the evolved stellar population are well constrained with the IRAC and MIPS bands. Furthermore MIPS shares two bands with PACS at 70 and $160 \mu \mathrm{m}$. This is important to compare the two instruments and assure that they correspond and to understand cases of disagreement (Rémy-Ruyer et al. 2013a, in preparation). Spitzer IRS spectroscopy, covering 5 to $35 \mu \mathrm{m}$, exists for all but 5 of the DGS galaxies and have been recently reprocessed (Rémy-Ruyer et al. 2013b, in preparation). The IRS spectroscopy covers 
the PAHs bands as well as many ionized lines which are relatively bright in dwarf galaxies and are thus important to constrain the properties of the ionizing sources and the nebular properties (e.g. Cormier et al. 2012; Lebouteiller et al. 2012). For example, MIR nebular lines, such as [NeII], [NeIII], [SIV], and [SIII], accessible with the Spitzer IRS, have relatively high critical densities $\left(>1000 \mathrm{~cm}^{-3}\right)$, complementing the PACS low density tracers, [NII], [NIII], and [OIII] lines (Table 1). Also, the 33 $\mu \mathrm{m}$ [SiII] line, which requires an ionization energy of $8.1 \mathrm{eV}$, complements the PACS neutral PDR gas tracers such as [CII] and [OI].

- The sensitivity of IRAS at 12, 25, 60 and $100 \mu \mathrm{m}$ (beam sizes: $10.8^{\prime} \times 4.5^{\prime}$ to 3.0' $\times 5.0^{\prime}$ ) provides data for about one half of the DGS sample (data from the IRSA site). The $12 \mu \mathrm{m}$ observations provide an additional valuable MIR constraint useful for modeling the global SEDs of the DGS galaxies.

- The DGS galaxies have also been detected by WISE at 3.4, 4.6, 12 and $22 \mu \mathrm{m}$ with spatial resolutions of $6.1 ", 6.4 ", 6.5 "$, and $12.0 "$, respectively, for all but one galaxy (data from the IRSA site). The $22 \mu \mathrm{m}$ observations, especially, will give the necessary constraint on the MIR wavelength range of the SED when neither IRAS nor Spitzer $24 \mu \mathrm{m}$ are available.

- Submm observations from ground-based telescopes are crucial to determine the presence and characterization of the submm excess. Usually this excess may begin to appear around 450-500 $\mu \mathrm{m}$ compelling observations longward of $500 \mu \mathrm{m}$. About $30 \%$ of the DGS sample have already been observed in the submm range with either the MAMBO $1.2 \mathrm{~mm}$ (IRAM - 30m telescope), LABOCA (APEX), SCUBA(JCMT) pr SCUBA-2 $(J C M T)$. New observations for DGS sources are currently underway with SCUBA-2 as well as with LABOCA on APEX to augment the submm observations to characterize and study the submm excess (Rémy-Ruyer et al. 2013b, in preparation). 
- Planck has observed the sky with bands from 30 to $857 \mathrm{GHz}$. Due to the relatively large Planck beams (4.5' to 33') and limited sensitivity, only a handful of galaxies are included in the Planck Early Release Catalog. Further Planck data processing in the next data-release in 2013 is expected to produce more results for the DGS targets.

A summary of the available ancillary data currently included in the DGS database, can be found in Table 5. Figure 4 gives a view of range of existing Herschel and ancillary for the DGS sample, and how many galaxies were detected for the different data sets. 







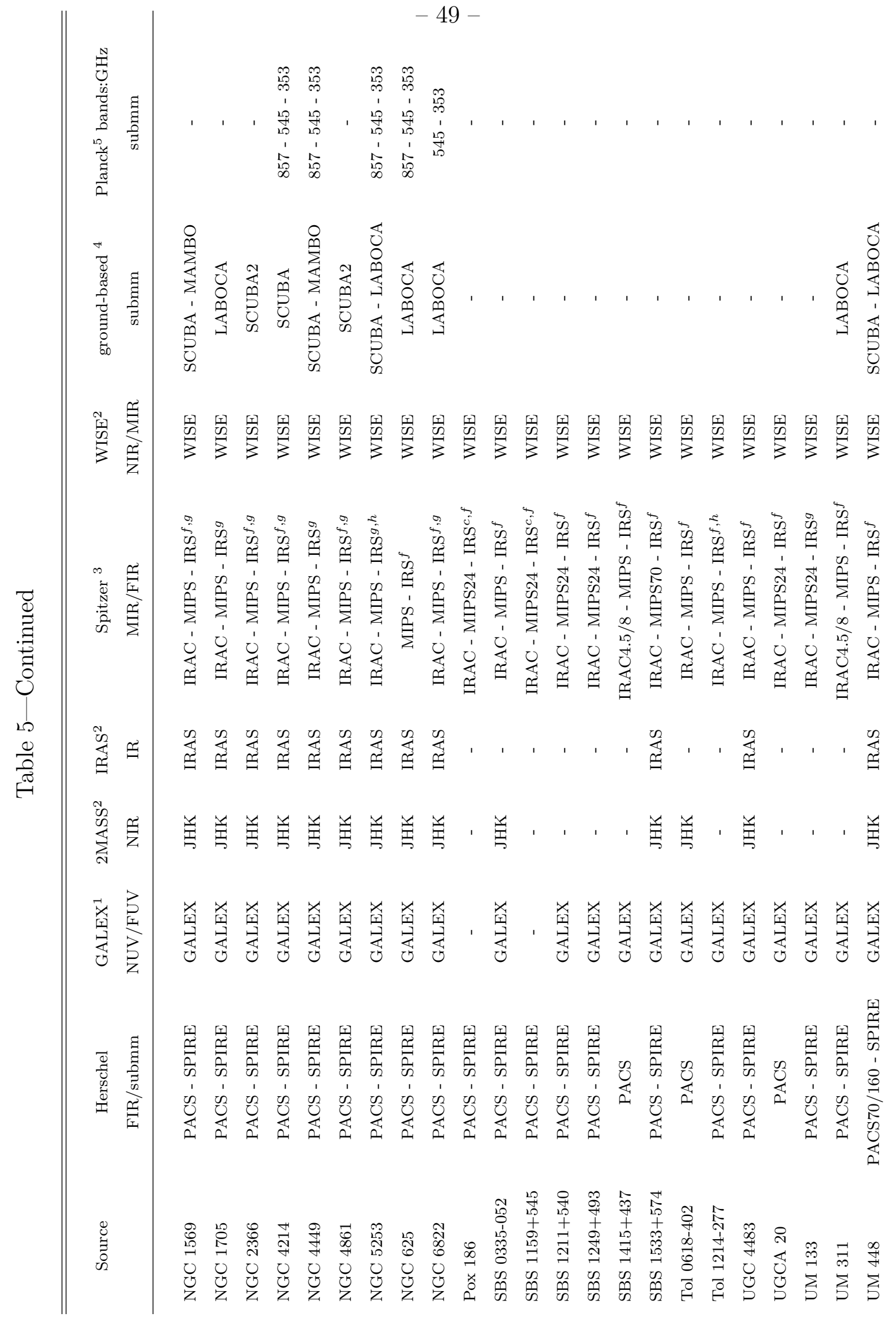




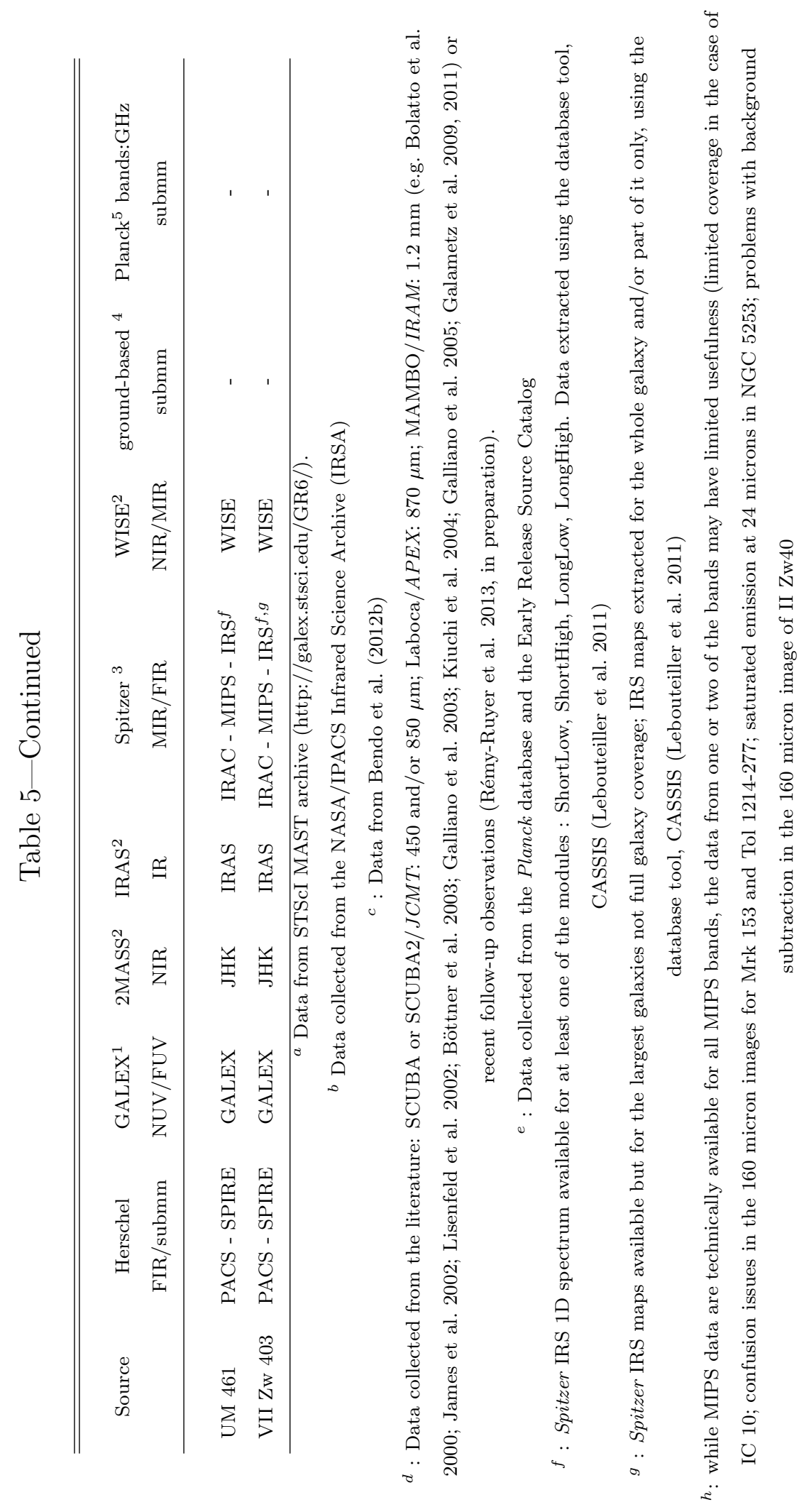




\section{A taste of science results}

Here we give some examples of science that can be carried out with the DGS, using the full photometric and spectroscopic Herschel data along with the available ancillary data at a variety of wavelengths.

\subsection{Distribution of the ISM components in NGC 4214}

An example of the detailed FIR and submm dust distribution that Herschel reveals is shown in Figure 6 for NGC 4214, a nearby (D=2.9 Mpc) star-forming dwarf galaxy with metallicity $12+\log (\mathrm{O} / \mathrm{H})=8.2($ Kobulnicky \& Skillman 1996). The PACS and SPIRE images from the DGS are the highest spatial resolution data at these wavelengths. The distribution of the dust traced by the 3 FIR PACS bands, where the peak of the total luminosity exists, resembles, at first glance, that of the longer wavelength SPIRE bands, which are mostly tracing the overall colder dust component. There are two particularly prominent star-forming regions seen at Spitzer and Herschel images: the more northern of these 2 prominent peaks is called central (C), while the peak just to the south-east of this is called SE. The quiescent extended "neck" region to the north-west is called NW. The $\mathrm{C}$ and SE sites contain clusters of widely varying ages and characteristics (Úbeda et al. 2007a; Fanelli et al. 1997) are very prominent at all PACS bands and at least up to 350 $\mu \mathrm{m}$ and are sitting in a more extended, diffuse dust component. These 2 Herschel peaks are seen prominently in $\mathrm{H} \alpha$ (Figure 6): the massive central HII region harboring a range of clusters overall $\sim 3$ to 4 Myr (MacKenty et al. 2000) and as well a super star cluster; the SE site being the second most prominent $\mathrm{H} \alpha$ region configured in a series of knots of some of the youngest clusters in the galaxy, $\sim 2$ Myr (Úbeda et al. 2007b). The NW region is characterized by a more quiescent cluster of $\mathrm{H} \alpha$ knots, also apparent in Herschel observations. The contrast between the bright star forming regions and the extended low 
surface brightness emission decreases toward longer wavelength, where, for example, at 500 $\mu \mathrm{m}$ the emission is rather dominated by the more extended cooler dust emission.

At other wavelengths, there are some obvious similarities to the Herschel data. In Figure 6, we include maps from the rich ancillary dataset available for NGC4214: GALEX tracing the youngest stellar components, $\mathrm{H} \alpha$ showing the distribution of the ionized gas, IRAC $3.6 \mu \mathrm{m}$ shows the evolved stellar component, IRAC $8 \mu \mathrm{m}$ the PAHs and/or hot dust component, MIPS $24 \mu \mathrm{m}$ (Bendo et al. 2012b) tracing the warm dust component and 21 cm HI, from the THINGS survey (Walter et al. 2008). Note how extended the HI emission is, while the star formation activity is primarily concentrated toward the central 2 star forming regions (C and $\mathrm{SE})$. 


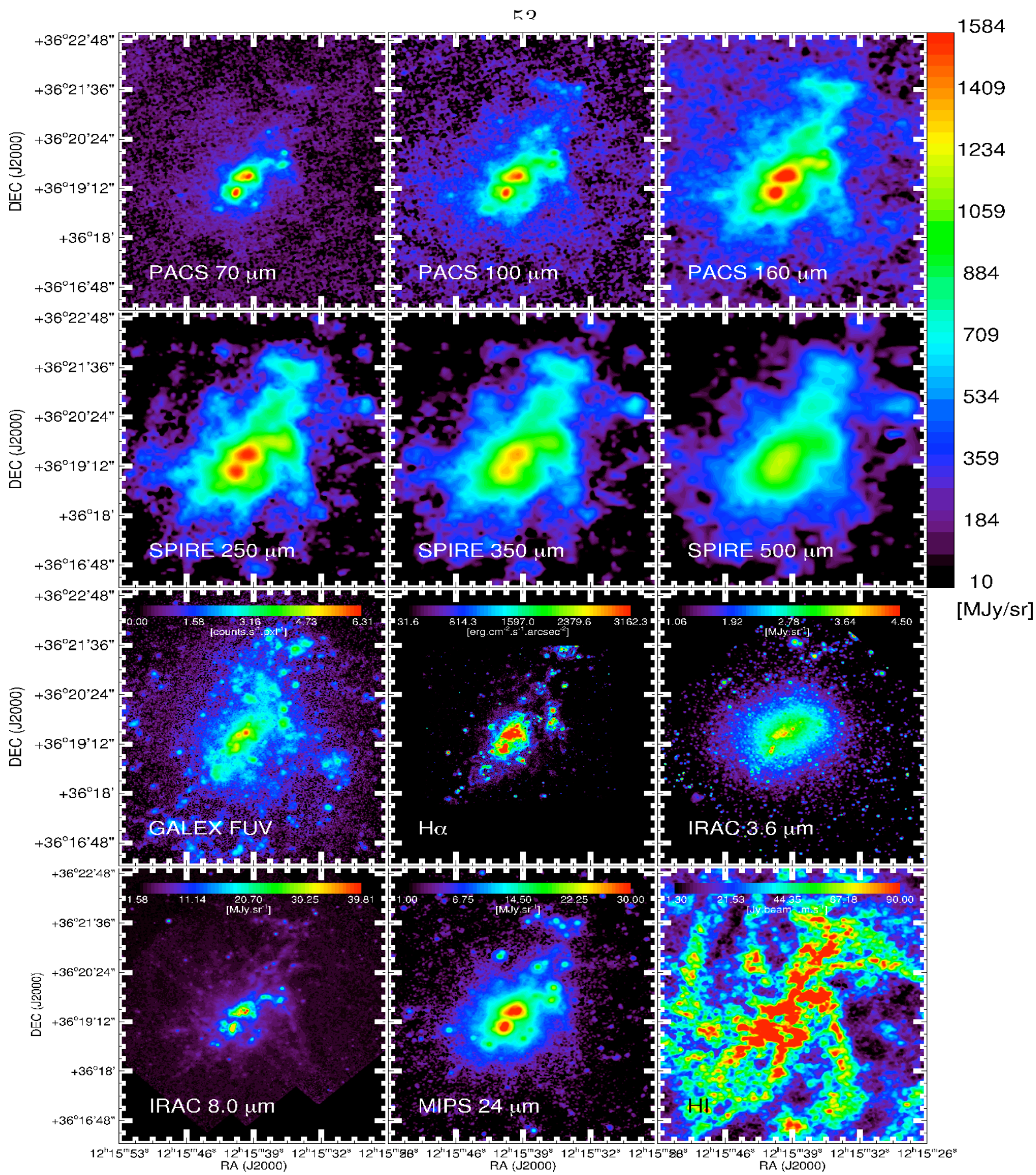

Fig. 6. - Herschel and ancillary images for the DGS galaxy, NGC4214. The PACS maps have been reduced with Scanamorphos and the SPIRE maps with a customized version of the official pipeline (see Rémy-Ruyer et al. 2013a, in preparation). Note the 2 star forming regions especially prominent at PACS FIR wavelengths: NGC4214-C is the more northern of the 2 brightest star forming regions, and NGC4214-SE is just south-east of this. NGC4214-C harbors a super star cluster. The rather quiescent extended "neck" region to the north-west is called NW. 

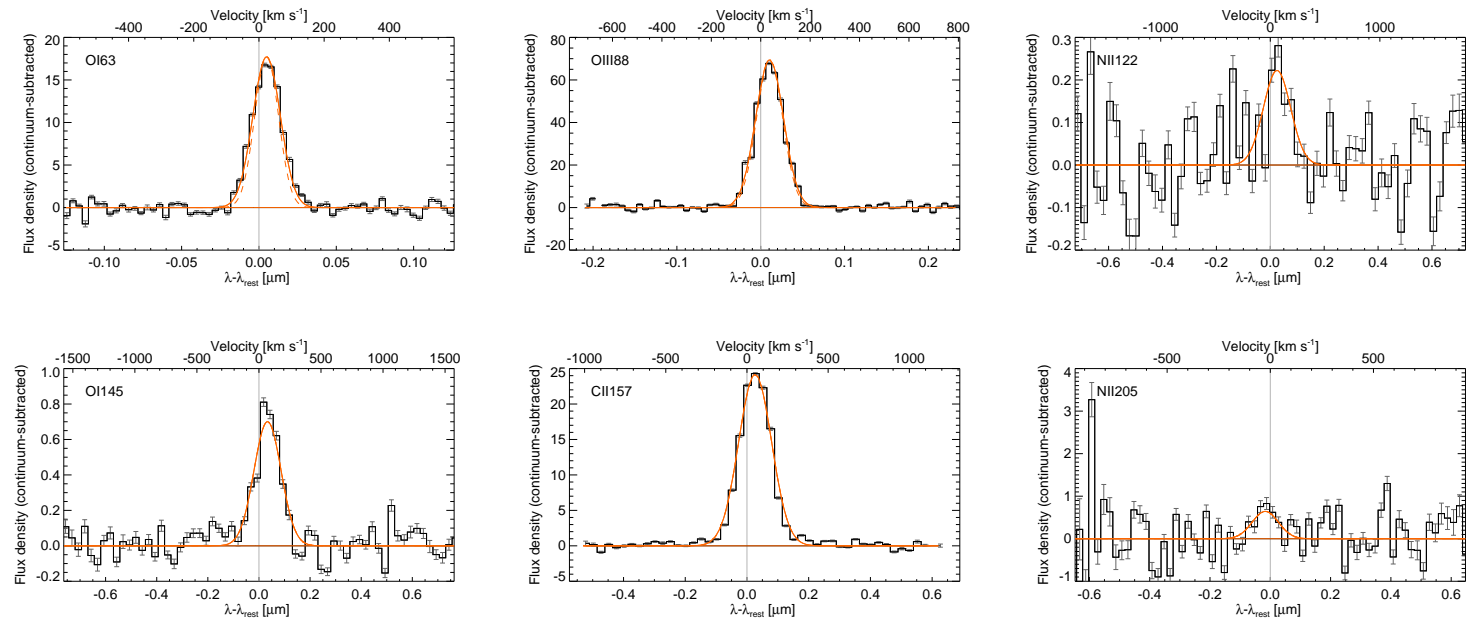

Fig. 7. - Example of the PACS spectral line quality observed near the central star forming region in NGC 4214. For these cases the lines are not resolved and the spectra have been binned to $1 / 5$ of the instrument spectral resolution (see section 4.2). 

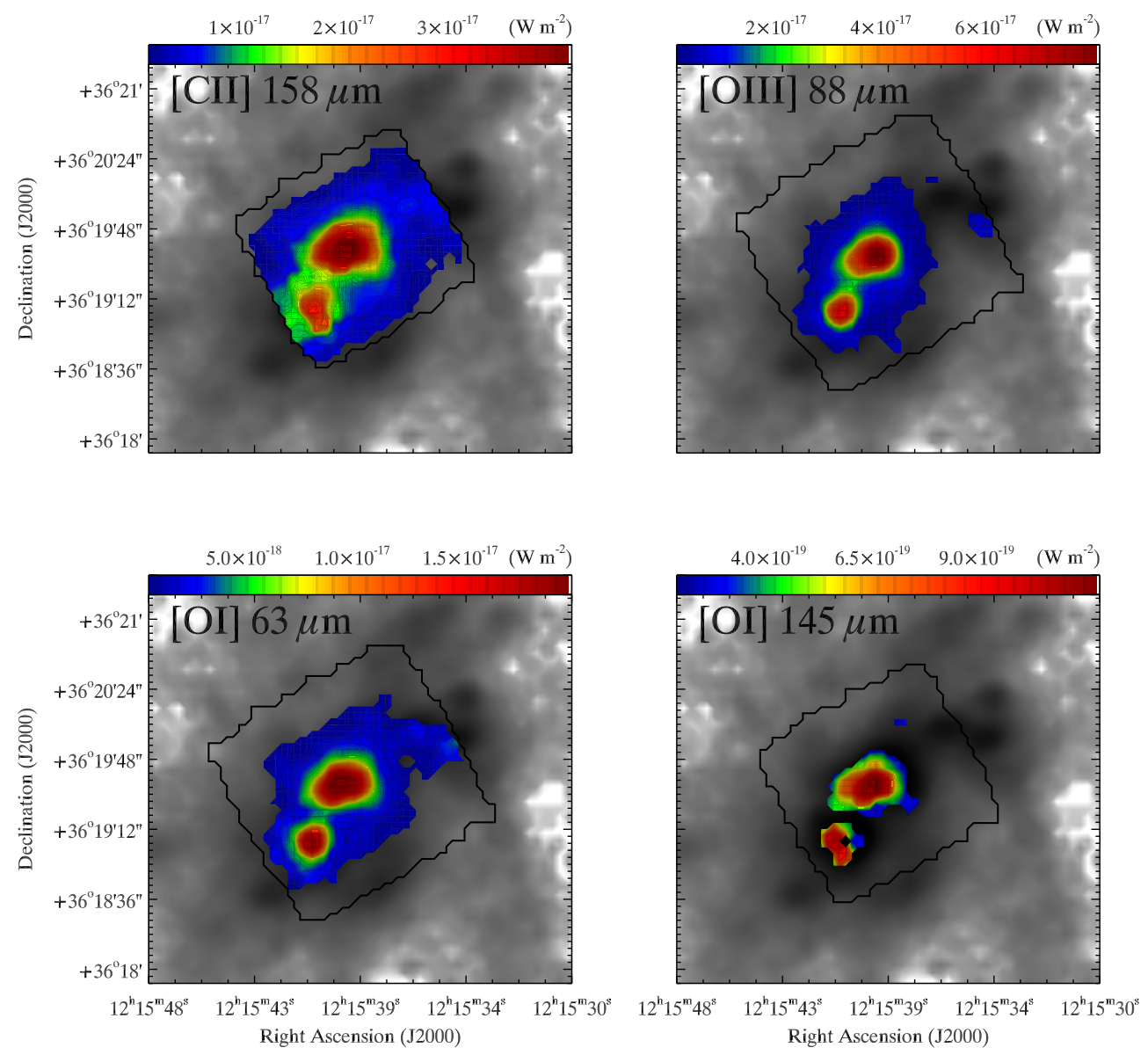

Fig. 8. - PACS spectroscopic maps on the $160 \mu \mathrm{m}$ photometry map of NGC 4214. The [CII] peaks are very broad, covering the star forming regions ( $\mathrm{C}$ and $\mathrm{SE}$ ) and the associated extended PDRs. The [OIII] line emission is much more peaked toward region $\mathrm{C}$, peaking on the location of the super star cluster, the ionizing source, whereas, the [OI] emission peaks toward $\mathrm{C}$ and SE, but the peak at $\mathrm{C}$ is shifted from the [OIII] peak (Cormier et al. 2010). The NW region is illuminated in the PDR tracers, [CII] and [OI]. 


\subsection{FIR dust and gas properties within NGC 4214}

Four PACS lines are very bright throughout NGC 4214: [OIII] 88 $\mu \mathrm{m}$, [CII] 158 $\mu \mathrm{m}$ and the [OI] 63 and $145 \mu \mathrm{m}$ lines (Figure 7). The [OIII] $88 \mu \mathrm{m}$ line is the brightest everywhere, not the [CII] $158 \mu \mathrm{m}$ line, which is most often the brightest line throughout normal metallicity galaxies. The very porous ISM in NGC4214, typical of low metallicity star forming galaxies, is very evident in the extent of the wide-scale distribution of [OIII] 88 $\mu \mathrm{m}$, requiring the presence of $35 \mathrm{eV}$ photons, consistent with the deficit of PAHs found on galaxy-wide scales, presumably destroyed by the permeating unattenuated UV photons in dwarf galaxies (e.g. Engelbracht et al. 2005; Madden et al. 2006). The porosity of NGC 4214 and other dwarf galaxies is also shown by studies concluding the importance of the escaping ionizing radiation on the dust SED (Hermelo et al. 2013; Cormier et al.|2012). The [NII] 122 and $205 \mu \mathrm{m}$ lines are also detected toward the peak star forming regions, but are 1 or 2 orders of magnitudes fainter than the other lines. All of the lines peak toward the 2 main star forming regions (Figure 8): the [OIII] peak coincides with the location of the super star cluster in region $\mathrm{C}$, while the $[\mathrm{CII}]$ peak is shifted off of the super star cluster and coincides with the peak of the neutral PDR tracers, the 2 [OI] lines. The $\mathrm{S} / \mathrm{N}$ of the [CII] map is remarkable - more than 10 throughout the region mapped as illustrated in Figure 9 . allowing for detailed spatial studies of the heating and cooling of the near and beyond the low metallicity star forming regions.

To illustrate the kind of spectral and spatial studies that can be carried out on the gas and dust properties, we present the combined gas and dust SEDs of the 3 main HII regions, created from Spitzer IRAC, MIPS, IRS and Herschel observations (Figure 10). The data treatment and dust modelling details are described in Rémy-Ruyer et al. (2013a, in preparation). The shapes of the dust SEDs are representative of typical compact HII regions. These SEDs include the MIR and FIR lines from the PACS spectroscopic maps 
to present the full gas plus dust SEDs. Note the relatively wide diversity of behavior of the high excitation vs. low excitation lines and the variation in the line to FIR continuum levels. For example, the NW region shows a much more quiescent dust SED as well as low levels of high excitation ionic lines, which are in contrast to the those of the 2 bright star forming regions at the origin of the peaks of the warm dust emission (C and SE). Notice that toward the central and south-eastern star forming regions, the PDR tracers, [CII] 158 $\mu \mathrm{m}$ and [OI] $63 \mu \mathrm{m}$ lines, as well as the ionized gas tracer, [OIII] $88 \mu \mathrm{m}$, dominate the infrared line emission and account for 2 to $4 \%$ of the total $L_{T I R}$ (Cormier et al. 2010). The rich MIR wavelength range shows prominent high excitation ionic lines, such as [NeII] $12.8 \mu \mathrm{m},[\mathrm{NeIII}] 15.5 \mu \mathrm{m}$, [OIV] $25.9 \mu$ mand [SIV] $10.5 \mu \mathrm{m}$, arising from the rather dense HII regions as well as the PAH bands, emitting from 3 to $17 \mu \mathrm{m}$. Cormier et al. (2012) demonstrate the level of complexity possible now in modeling $\sim 20$ MIR and FIR fine structure lines self-consistently with the dust continuum.

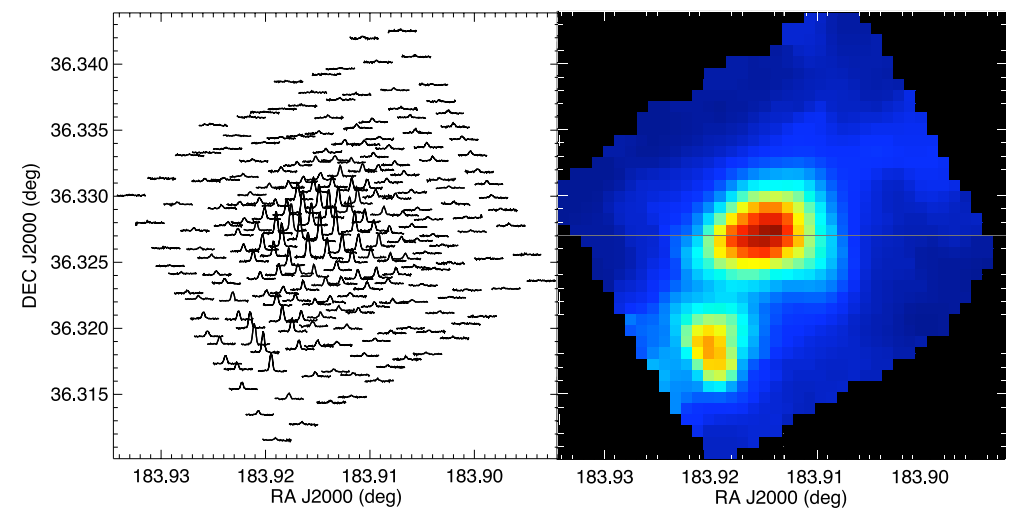

Fig. 9.- (left): Distribution of the PACS [CII] spectra. Even toward the edges of the region mapped $\left(2^{\prime} \times 2^{\prime}\right)$, the $\mathrm{S} / \mathrm{N}$ of the $[\mathrm{CII}]$ line is at least 10 . (right) Image of the [CII] line map, showing the peak emission toward regions $\mathrm{C}$ and $\mathrm{NE}$. 


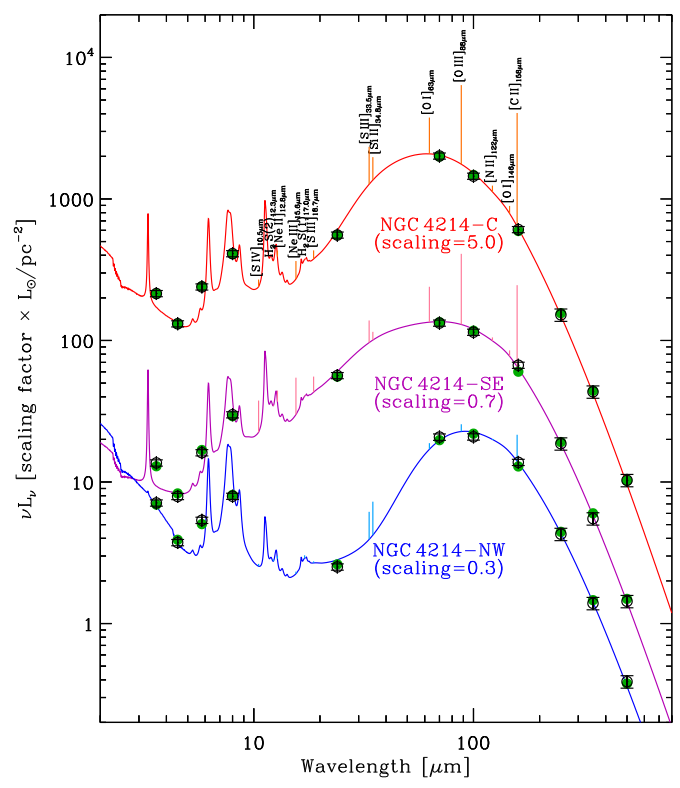

Fig. 10.- SEDs of the 2 main star forming regions, in the center (NGC4214-C) and to the southeast (NGC4214-SE) along with the SED of a more quiescent region to the north-west (NGC4214NW) for comparison. For clarity, the luminosities have been multiplied by the scaling factor indicated below each SED. The black circles with error bars are the integrated Spitzer and Herschel broadband observations. The solid lines are the fitted SED models (Galliano et al.|2011), fit to the broadband observations. The green dots are the synthetic photometry of the model. The vertical spectra on top of the SED represent the observed integrated line luminosities from Spitzer IRS and Herschel PACS (Cormier et al. 2010). 


\subsection{Submillimetre excess}

In Figure 11 we present examples of modelled SEDs for the new Herschel data as well as with the ancillary dataset available for VIIZw403 and NGC 1569. From these modeled SEDs we will have access to new parameters for the galaxies, such as the total dust mass, total luminosity, new SFR and dust composition and dust size distribution (Rémy-Ruyer et al. 2013a, 2013b in preparation). Comparison of the observed SEDs with modeled SEDs in dwarf galaxies often points to an as-yet inexplicable excess (Section 2) which begins to become apparent near $\sim 500 \mu \mathrm{m}$, the longest wavelength observed by Herschel, or sometimes becomes more evident at longer submm wavelengths, in which case it may be detectable with ground-based submm telescopes, such as JCMT(SCUBA2), $A P E X(\mathrm{LABOCA}), \operatorname{IRAM(GISMO)}$ or $A L M A$, for example. VIIZw403 already shows a submm excess beginning at $\sim 500 \mu \mathrm{m}$ (Figure 11). In NGC 1569, on the other hand, there is no hint of submm excess throughout the Herschel SPIRE bands, including $\sim 500 \mu \mathrm{m}$. However, a small excess beginning at $\sim$ SCUBA $850 \mu \mathrm{m}$ observations is present, growing at mm wavelengths (Galliano et al. 2003). In this case, the submm excess would not be detectable without wavelengths beyond Herschel. The Herschel data are crucial to properly constrain the submm dust emission to be able to accurately extrapolate and interpret the SED to wavelengths $>500 \mu \mathrm{m}$. Without the Herschel data, the Rayleigh-Jeans side of the dust SED is poorly constrained, making it more difficult to properly assess whether the submillimetre excess is present. Accurate quantification of the submm excess at SPIRE wavelengths must take into account careful consideration of the most recent calibration and beam sizes in the aperture photometry.

Note that these are global SEDs presented here, and spatial SED modelling throughout well-resolved galaxies, may uncover regions where the presence of this submm excess may be apparent even at Herschel wavelengths, as has been detected with SPIRE within the 
LMC, where the presence of this excess is anti-correlated with the dust surface density (Galliano et al. 2011). 

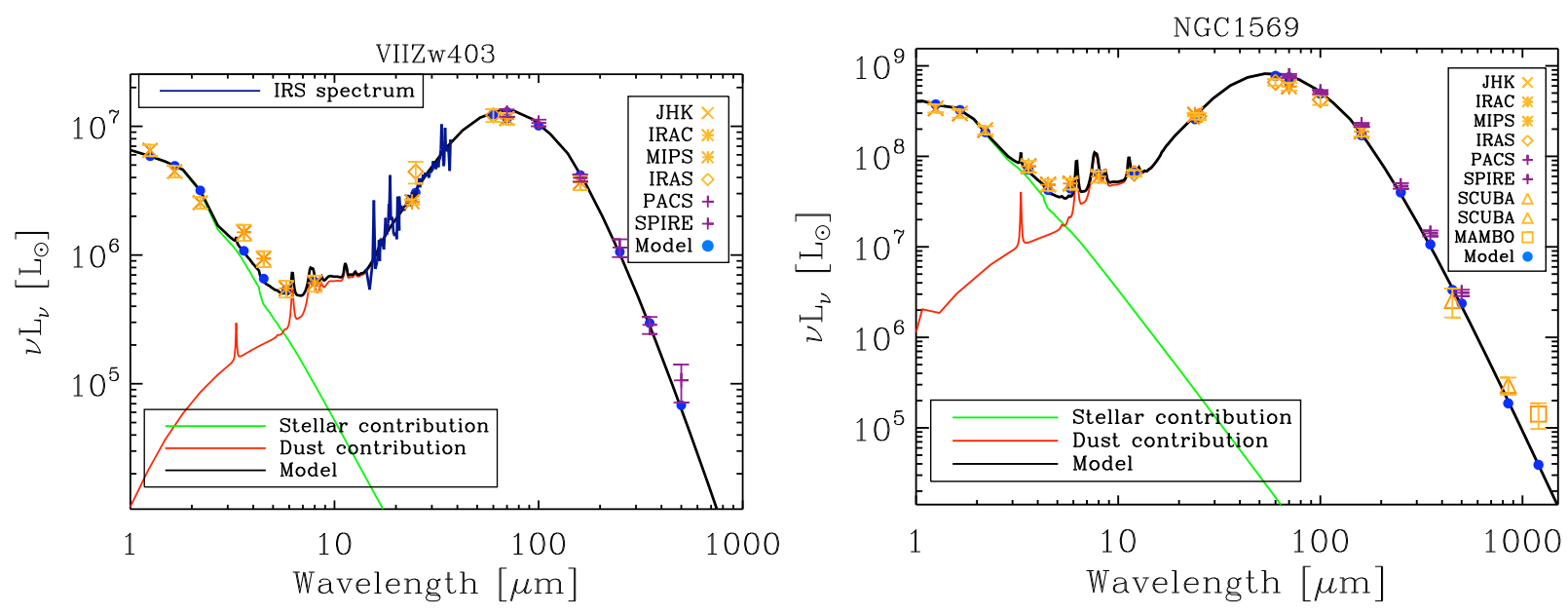

Fig. 11. - Examples of galaxy-wide SEDs for two DGS galaxies : VIIZw403 (left), and NGC 1569 ( right). Both SEDs have been modelled as in Galliano et al. (2011) and include the new Herschel data (purple) as well as any available ancillary data (orange). The different symbols refer to the different instruments that have been used. For VIIZw403, the IRS spectrum is displayed in dark blue. While limited IRS spectra do exist for NGC1569, we do not include them in this SED fit, due to lack of full galaxy coverage. The total modelled SED in black is the sum of the contributions displayed here: stellar (green) and dust (red). The model points in the different considered bands are the filled blue circles. The dust SED has been corrected for CO line contamination as well as free-free emission. Notice the submm excess that becomes apparent in VIIZw403 at the SPIRE 500 $\mu \mathrm{m}$ band while some galaxies, such as NGC 1569, for example, the submm excess may only begin at longer wavelengths, such as 850/870 $\mu \mathrm{m}$ or longer (Galliano et al. 2003; Galametz et al. 2009, Rémy-Ruyer et al. 2013a, in preparation). 


\section{DGS Products}

The final products for the PACS and SPIRE photometry data will become public on the HeDaM database (http://hedam.oamp.fr/; (Roehlly et al. 2011)) by the end of 2013/beginning of 2014. All of the Herschel maps for the DGS galaxies will be available in FITS format as well as Jpg format. A catalog of the new Herschel fluxes will also be available in Rémy-Ruyer et al. 2013a, in preparation. Since the PACS and SPIRE spectroscopic data is still in the process of being finalized, this data is forseen to become available in mid-2014. This data will be delivered in the form of final total spectra and total flux values.

As an illustration of the wealth of data the DGS brings, in Appendix A we have assembled an Atlas of 3 color images of the DGS composed of Spitzer MIPS $24 \mu \mathrm{m}$, tracing the hot dust component, Herschel PACS $100 \mu \mathrm{m}$, close to the peak of the dust SED, and Herschel $250 \mu \mathrm{m}$, tracing the cooler dust.

\section{Summary}

We present an overview of the Dwarf Galaxy Survey which has used 230h of Herschel observations to study the FIR and submm gas and dust properties in 50 star forming dwarf galaxies of the local Universe with 6 PACS and SPIRE bands at 70, 100, 160, 250, 350, $500 \mu \mathrm{m}$ and the PACS and SPIRE spectrometers. The PACS FIR spectroscopy gives us maps of [CII] $158 \mu \mathrm{m}$, [OI] 63 and $145 \mu \mathrm{m}$, [OIII] $88 \mu \mathrm{m}$, [NIII] $57 \mu \mathrm{m}$ and [NII] 122 and $205 \mu \mathrm{m}$ The SPIRE FTS covers 194 to $671 \mu \mathrm{m}$ and includes high-J CO lines (J=4-3 to $\mathrm{J}=13-12)$, [NII] $205 \mathrm{mu}$ and [CI] lines at 370 and $609 \mu \mathrm{m}$. The targets span a wide range of metallicity values, as low as $1 / 50 \mathrm{Z}_{\odot}$, and cover 4 orders of magnitude of star formation rates. The full DGS database goes beyond the FIR and submm observations of Herschel, 
and includes a rich array of ancillary observations, including Spitzer MIR photometry and spectroscopy and FIR photometry, 2MASS NIR data, WISE MIR data, GALEX UV and FUV observations, IRAS, and ground-based submm data, for example. We present some science goals the full survey will bring and show some examples to illustrate the potential of this survey for futures studies of the distribution of the gas and dust properties within galaxies as well as on global scales. We anticipate that advances on the evolution of the dust and gas as a function of metallicity will bring us closer to understanding the enrichment of the Universe over cosmic times. 


\section{A. Determination of Metallicities}

The metallicity of a galaxy is an intrinsic parameter tracing the evolution of the system: the ISM becoming more enriched with elements as a function of age. Calibrating this important parameter from observations and models has lead to discrepancies that have been studied extensively (see Kewley \& Ellison (2008) for a review on this subject). Here we compare the results in determining the metallicity values of the DGS sample using the method of Izotov et al. (2006) (I06), who determine the electron temperature, $\mathrm{T}_{e}$, from the [OIII] $\lambda 4363$ line with the method of Pilyugin \& Thuan (2005) (PT05) which uses the $\mathrm{R}_{23}$ ratio : $\mathrm{R}_{23}=([\mathrm{OII}] \lambda 3727+[\mathrm{OIII}] \lambda \lambda 4959,5007) / \mathrm{H} \beta$. Degeneracies in the upper and lower branches of this calibration are removed using the [NII] $] 6584 /[\mathrm{OII}] \lambda 3727$ ratio (Kewley \& Ellison 2008). In Table 6 we provide the metallicity values of the DGS targets determined

for the I06 method. Uncertainty values have been determined by propagating the errors in the observed lines from the literature. Table 2 includes the metallicity values of the PT05 method, which has been adopted for the DGS survey.

Figure 12 shows the variation between the I06 and PT05 methods of metallicity calibration. We find a mean deviation of \pm 0.1 dex, as shown by the dashed lines. 


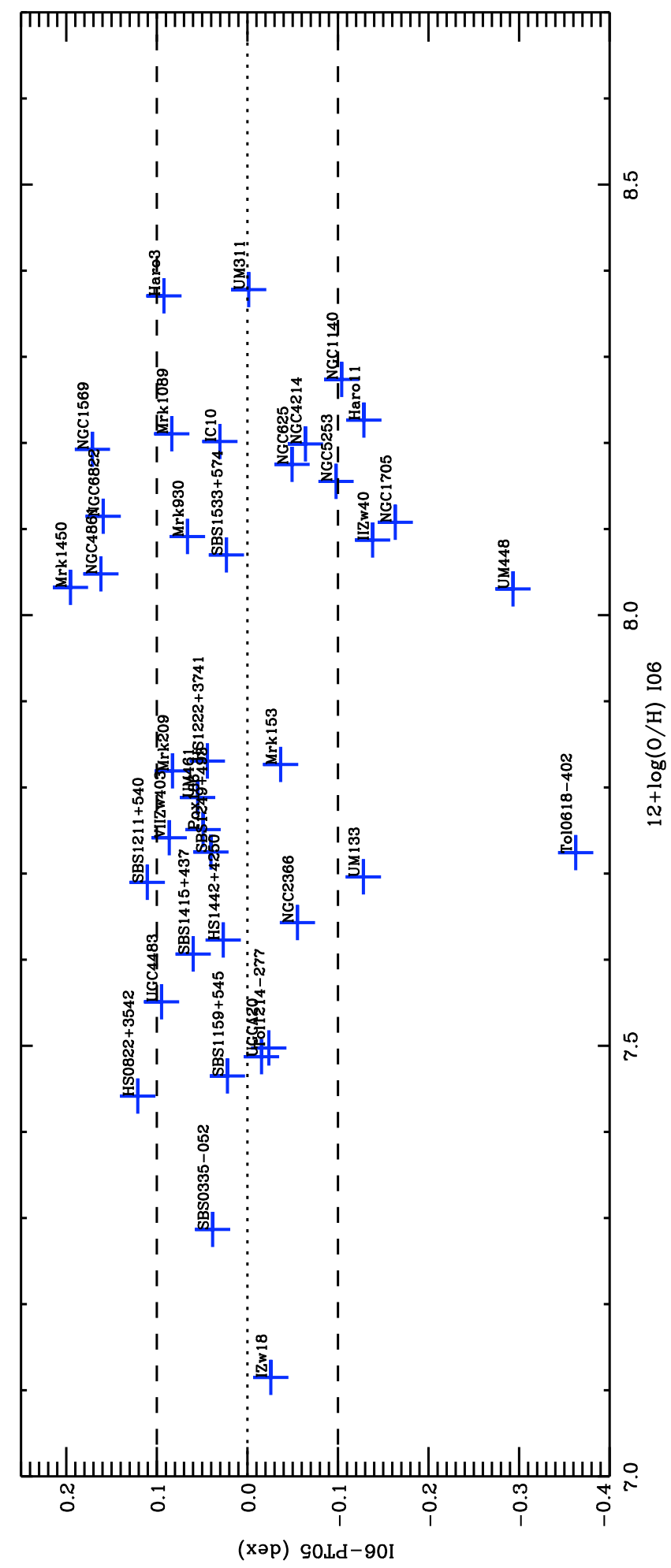

Fig. 12.- Comparison of I06 and PT05 metallicity calibrations for the DGS galaxies. The dashed lines delineate the mean variation of the sample, \pm 0.1 dex. 
Table 6. Metallicities for the DGS galaxies determined using the I06 calibration

\begin{tabular}{|c|c|c|}
\hline Source & $12+\log (\mathrm{O} / \mathrm{H})(\mathrm{I} 06)$ & Ref \\
\hline Haro11 & $8.23 \pm 0.03$ & 1 \\
\hline Haro $^{a}$ & - & - \\
\hline Haro3 & $8.37 \pm 0.02$ & 3 \\
\hline $\mathrm{He} 2-10^{a}$ & - & - \\
\hline $\mathrm{HS} 0017+1055^{b}$ & $7.63 \pm 0.10$ & 5 \\
\hline $\mathrm{HS} 0052+2536^{b}$ & $8.07 \pm 0.20$ & 5 \\
\hline HSO822+3542 & $7.44 \pm 0.06$ & 6 \\
\hline HS1222+3741 & $7.83 \pm 0.03$ & 7 \\
\hline $\mathrm{HS} 1236+3937^{c}$ & $7.47 \pm 0.1$ & 8 \\
\hline $\mathrm{HS} 1304+3529^{c}$ & $7.66 \pm 0.1$ & 8 \\
\hline $\mathrm{HS} 1319+3224^{c}$ & $7.59 \pm 0.1$ & 8 \\
\hline $\mathrm{HS} 1330+3651^{c}$ & $7.66 \pm 0.1$ & 8 \\
\hline HS1442+4250 & $7.62 \pm 0.02$ & 9 \\
\hline $\mathrm{HS} 2352+2733^{b}$ & $8.40 \pm 0.20$ & 5 \\
\hline IZw18 & $7.12 \pm 0.04$ & 10 \\
\hline IIZw40 & $8.09 \pm 0.02$ & 11 \\
\hline IC10 & $8.20 \pm 0.06$ & 12 \\
\hline Mrk1089 $9^{d}$ & $8.39 / 8.21 \pm 0.12$ & 13 \\
\hline Mrk1450 & $8.03 \pm 0.01$ & 14 \\
\hline Mrk153 & $7.83 \pm 0.05$ & 15 \\
\hline Mrk209 & $7.82 \pm 0.01$ & 16 \\
\hline Mrk930 & $8.09 \pm 0.03$ & 17 \\
\hline NGC1140 & $8.27 \pm 0.08$ & 3 \\
\hline NGC1569 & $8.19 \pm 0.03$ & 18 \\
\hline NGC1705 & $8.11 \pm 0.13$ & 19 \\
\hline NGC2366 & $7.64 \pm 0.03$ & 20 \\
\hline NGC4214 & $8.20 \pm 0.03$ & 4 \\
\hline NGC4449 ${ }^{a}$ & - & - \\
\hline NGC4861 & $8.05 \pm 0.01$ & 16 \\
\hline NGC5253 & $8.16 \pm 0.03$ & 4 \\
\hline NGC625 & $8.18 \pm 0.03$ & 22 \\
\hline NGC6822 & $8.12 \pm 0.07$ & 23 \\
\hline
\end{tabular}




\section{Table 6 - Continued}

\begin{tabular}{lcc}
\hline \multicolumn{1}{c}{ Source } & $12+\log (\mathrm{O} / \mathrm{H})(\mathrm{I} 06)$ & Ref \\
\hline Pox186 & $7.75 \pm 0.01$ & 24 \\
SBS0335-052 & $7.29 \pm 0.01$ & 17 \\
SBS1159+545 & $7.46 \pm 0.02$ & 14 \\
SBS1211+540 & $7.69 \pm 0.01$ & 14 \\
SBS1249+493 & $7.73 \pm 0.01$ & 25 \\
SBS1415+437 & $7.61 \pm 0.01$ & 26 \\
SBS1533+574 & $8.07 \pm 0.02$ & 16 \\
Tol0618-402 & $7.72 \pm 0.02$ & 27 \\
Tol1214-277 & $7.50 \pm 0.01$ & 4 \\
UGC4483 & $7.55 \pm 0.03$ & 28 \\
UGCA20 & $7.49 \pm 0.04$ & 29 \\
UM133 & $7.70 \pm 0.01$ & 4 \\
UM311 & $8.38 / 8.36 \pm 0.05$ & $17,30,31$ \\
UM448 & $8.03 \pm 0.04$ & 17 \\
UM461 & $7.79 \pm 0.03$ & 15 \\
VIIZw403 & $7.74 \pm 0.01$ & 16 \\
\hline & Notes : &
\end{tabular}

${ }^{a}:$ For these galaxies, the $[\mathrm{OIII}] \lambda 4363$ line was not detected and a direct determination of the metallicity with the I06 method was therefore not possible.

${ }^{b}$ : For these galaxies, we quote Ugryumov et al. (2003) who use the [OIII] $\lambda 4363$ line to determine the electron temperature and the method from Izotov et al. (1994). I06 is an updated version of the method of Izotov et al. (1994).

${ }^{c}$ : For these galaxies, no line intensity uncertainties, from which the metallicities are determined, are quoted. We assume a conservative value of 0.1 which is the mean of the variation of the difference between PT05, and the I06 method.

$d$ : These galaxies are members of groups of galaxies. We quote the value of the metallicity for the individual galaxy (left value) and that for the whole group (right value), which is the mean of all of the metallicities in the group. For Mrk1089, the galaxy is region A-C from López-Sánchez \& Esteban (2010). For UM311, the galaxy is region 3 following Moles et al. (1994).

References for metallicities : (1) Guseva et al. (2012) ; (3) Izotov et al. (2004) ; (4) Kobulnicky et al. (1999) ; (5) Ugryumov et al. (2003) ; (6) Pustilnik et al. (2003) ; (7) Izotov et al. (2007) ; (8) Popescu \& Hopp (2000) ; (9) Guseva et al. (2003a) ; (10) Izotov \& Thuan (1999) ; (11) Guseva et al. (2000) ; (12) Magrini \& Gonçalves (2009) ; (13) López-Sánchez \& Esteban 2010 ; (14) Izotov et al. (1994) ; (15) Izotov et al. 2006) ; (16) Izotov et al. (1997) ; (17) Izotov \& Thuan (1998) ; (18) Kobulnicky \& Skillman (1997) ; (19) Lee \& Skillman 2004) ; (20) Saviane et al. (2008) ;

(22) Skillman et al. (2003) ; (23) Lee et al. (2006) ; (24) Guseva et al. 2007) ; (25) Thuan et al. (1995) ; (26) Guseva et al. (2003b) ; (27) Masegosa et al. (1994) ; (28) van Zee \& Haynes (2006) ; (29) van Zee et al. (1996) ; (30) Moles et al. (1994) ; (31) Pilyugin \& Thuan (2007) 


\section{B. DGS Spitzer-Herschel Atlas}

As an illustration of the wealth of data the DGS brings, we have assembled an atlas of 3-color images of the DGS galaxies composed of Spitzer MIPS $24 \mu \mathrm{m}$ (blue), tracing the hot dust component, PACS $100 \mu \mathrm{m}$ (green), close to the peak of the dust SED, and SPIRE $250 \mu \mathrm{m}$ (red), tracing the cooler dust. Note that the images here are produced using their original resolution. The beams for the different bands are indicated in the lower right of each image. When a band is not present in the 3-color image, the source was not observed at that wavelength. 

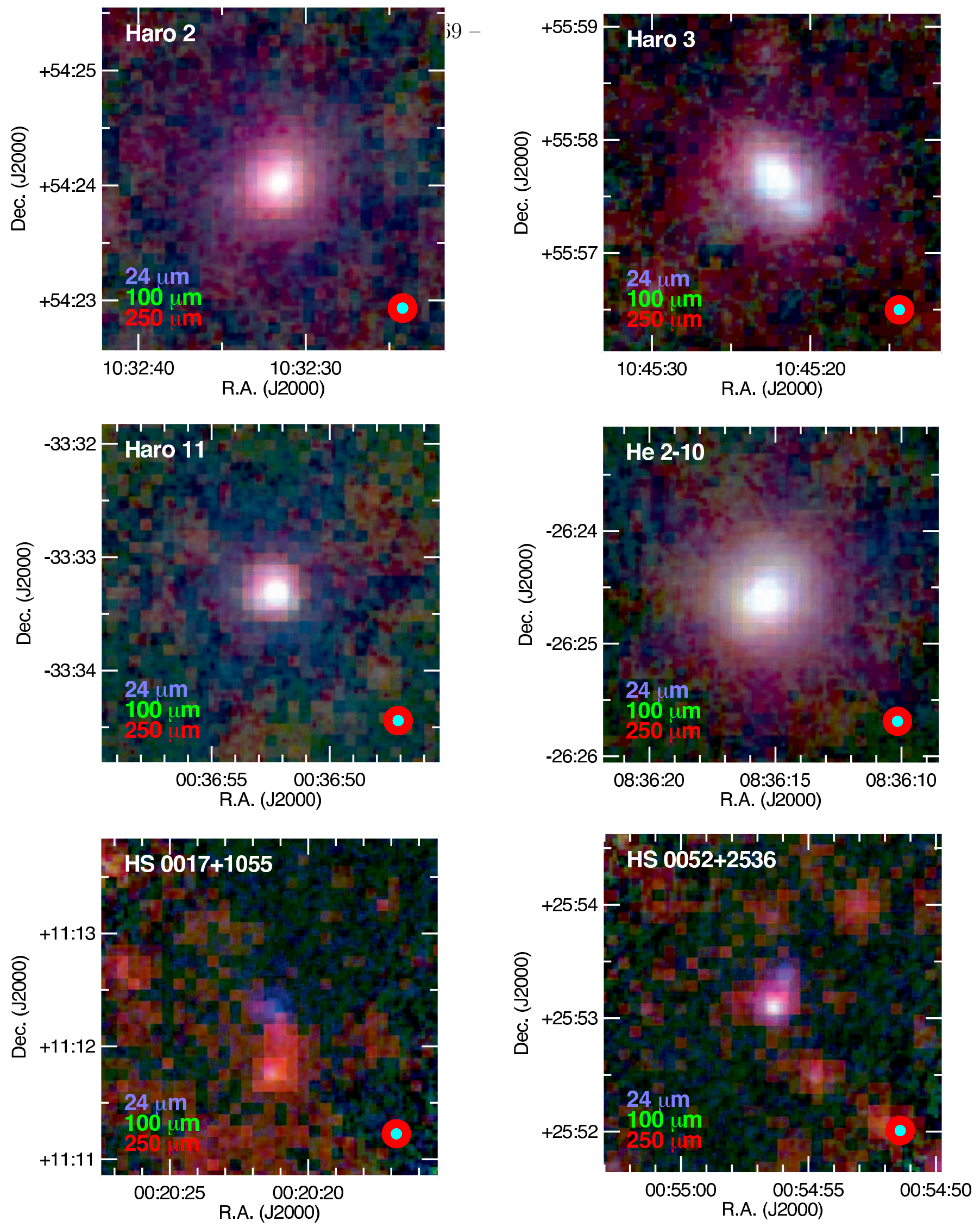

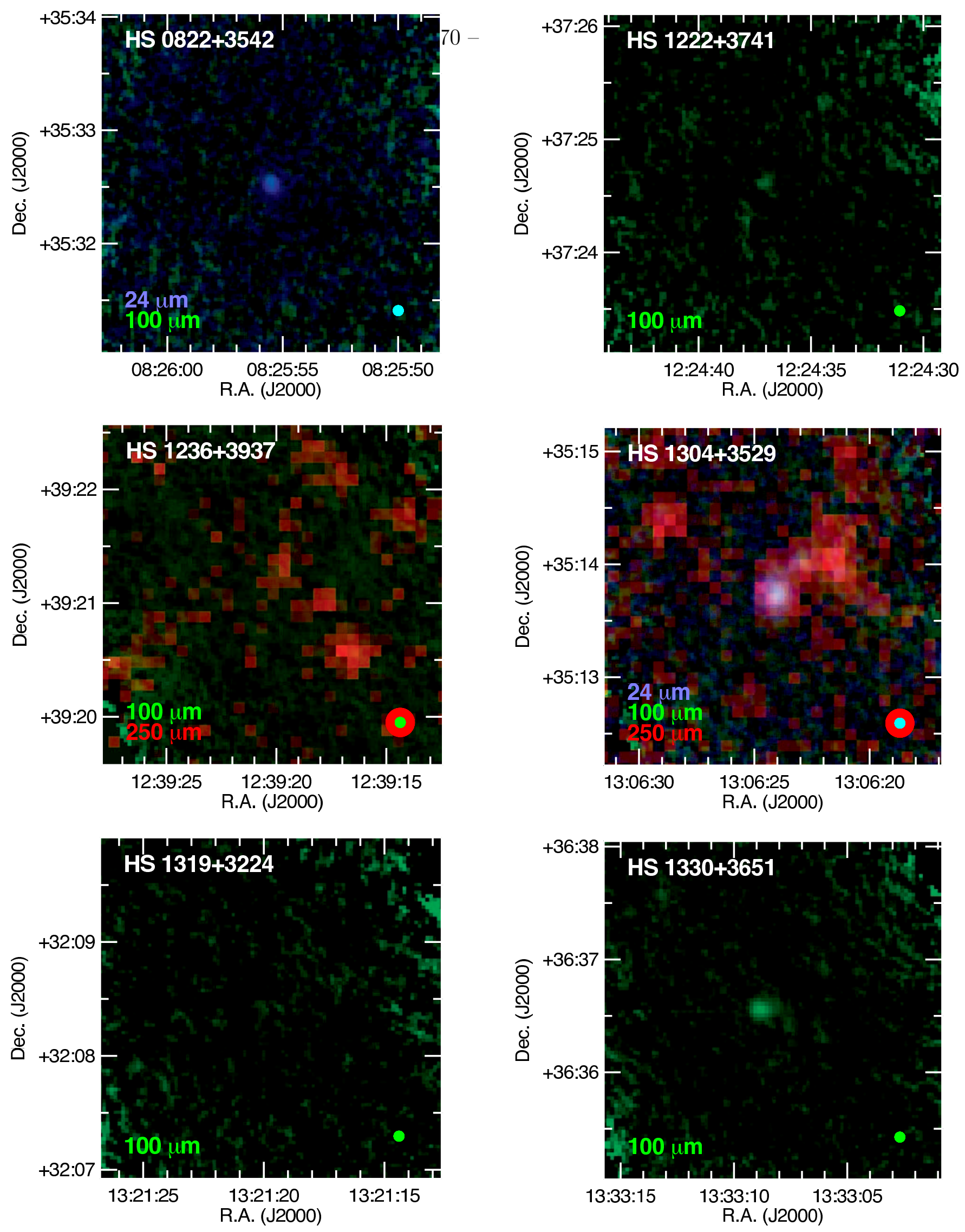

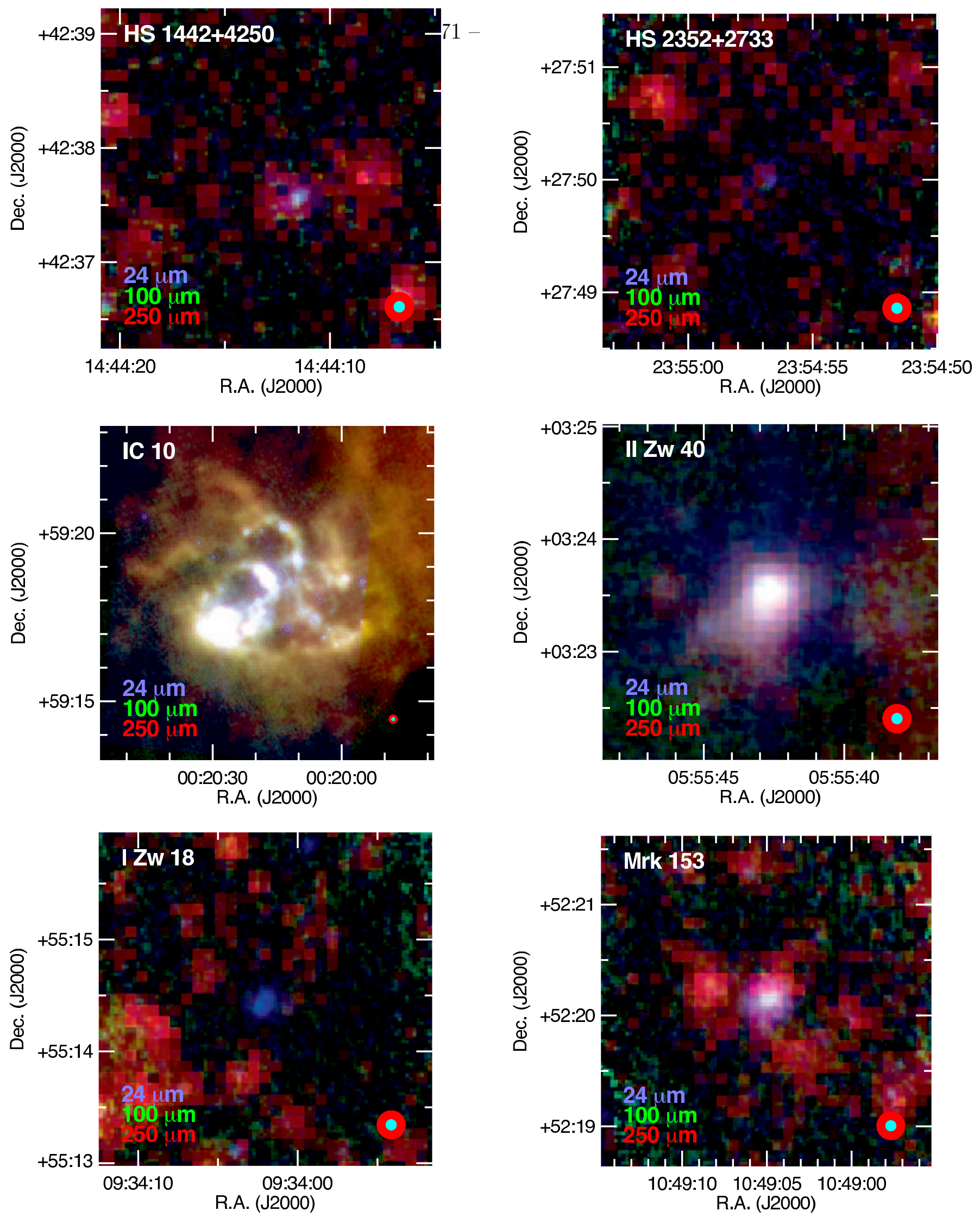

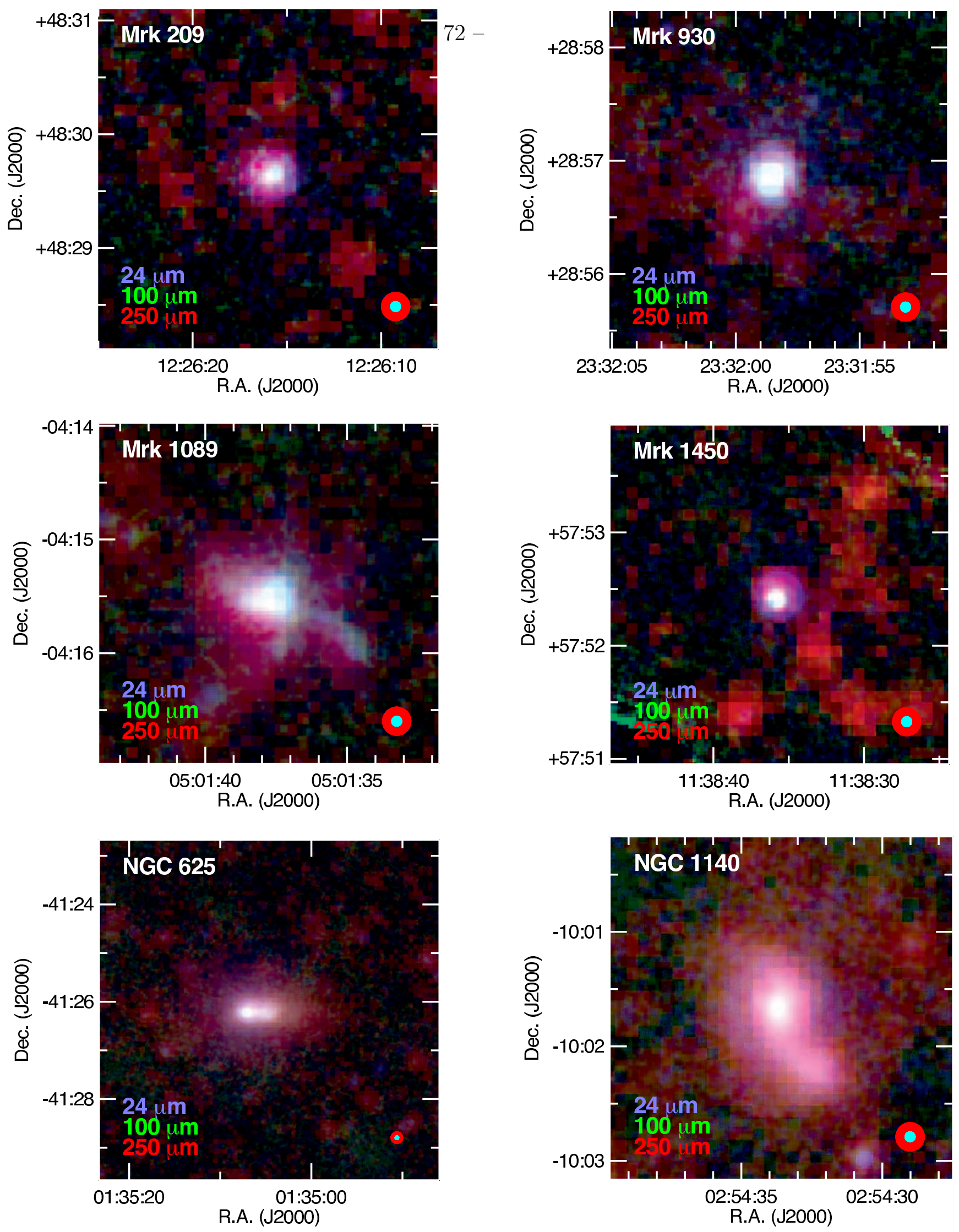



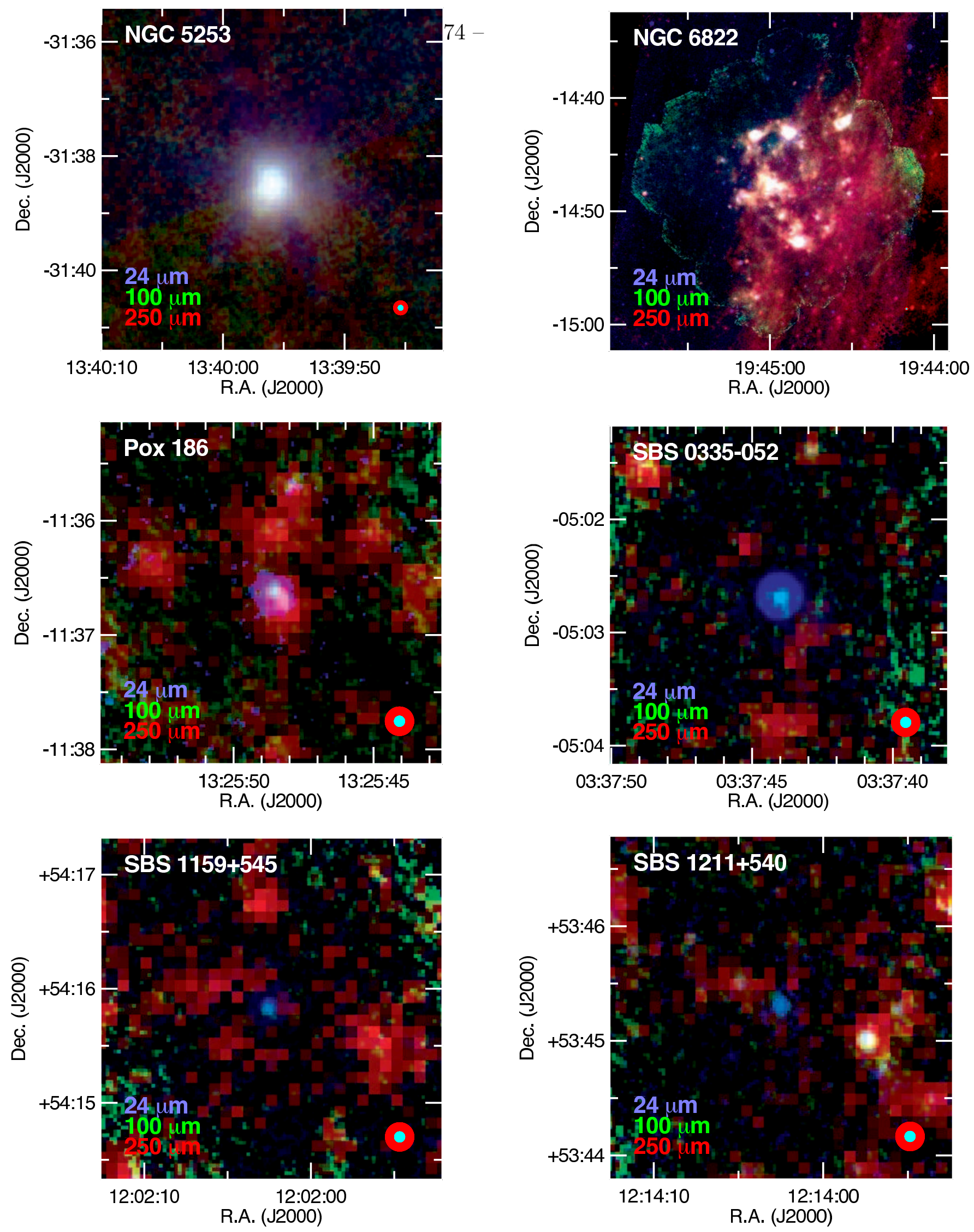

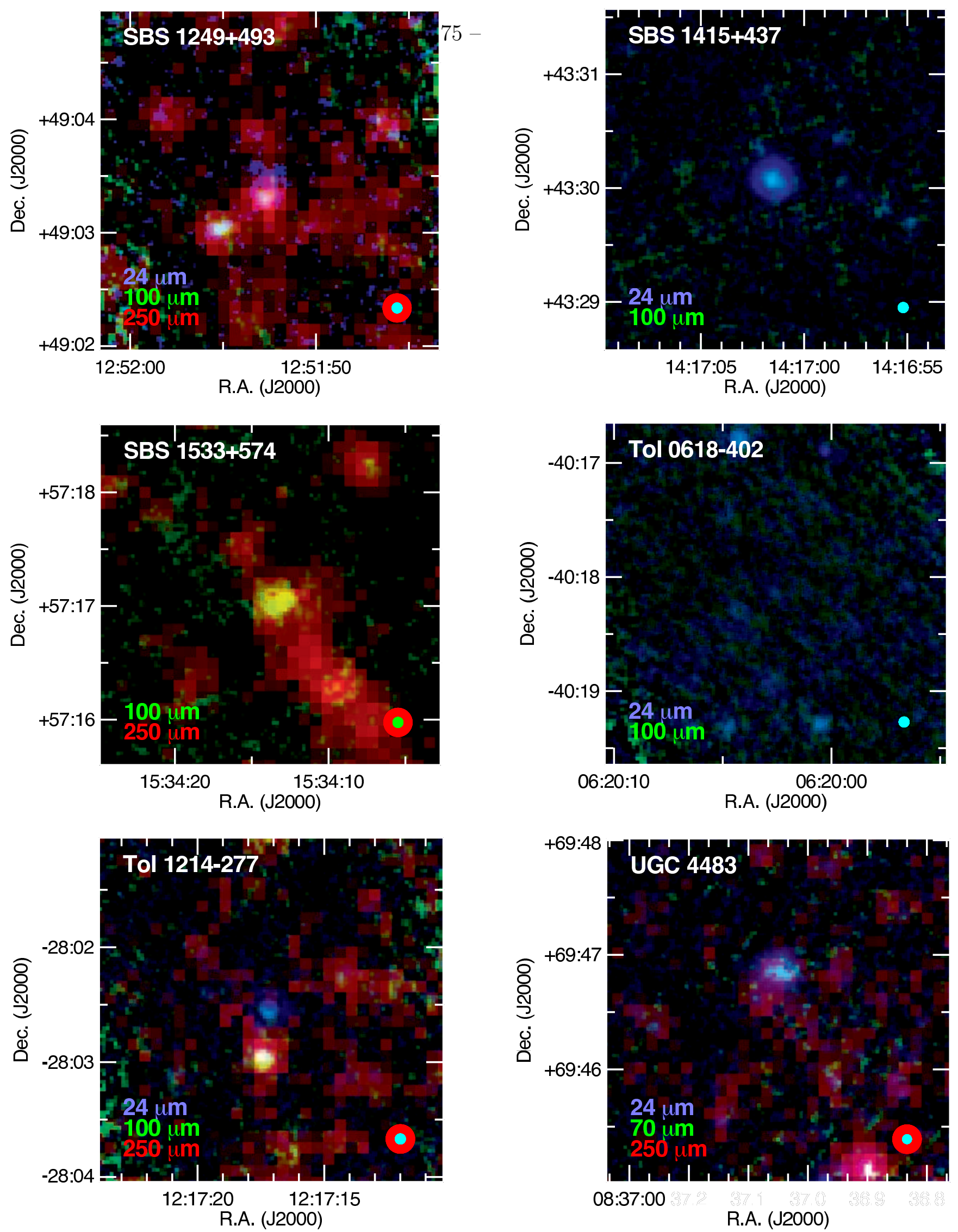

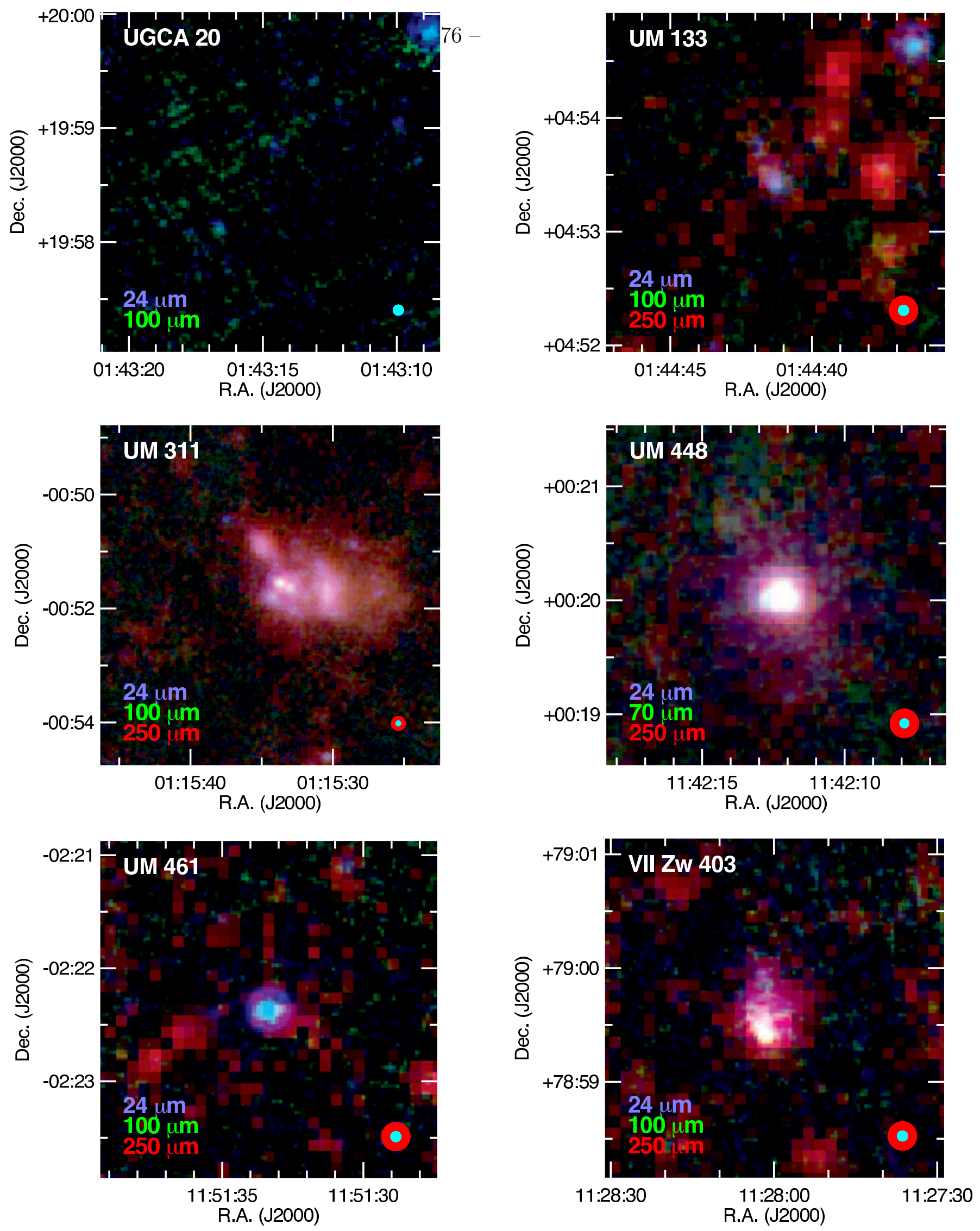
We thank the anonymous referee for useful suggestions which help to improve the clarity of the manuscript. PACS has been developed by MPE (Germany); UVIE (Austria); KU Leuven, CSL, IMEC (Belgium); CEA, LAM (France); MPIA (Germany); INAF-IFSI/OAA/OAP/OAT, LENS, SISSA (Italy); IAC (Spain). This development has been supported by BMVIT (Austria), ESA-PRODEX (Belgium), CEA/CNES (France), DLR (Germany), ASI/INAF (Italy), and CICYT/MCYT (Spain). SPIRE has been developed by Cardiff University (UK); Univ. Lethbridge (Canada); NAOC (China); CEA, LAM (France); IFSI, Univ. Padua (Italy); IAC (Spain); SNSB (Sweden); Imperial College London, RAL, UCL-MSSL, UKATC, Univ. Sussex (UK) and Caltech, JPL, NHSC, Univ. Colorado (USA). This development has been supported by CSA (Canada); NAOC (China); CEA, CNES, CNRS (France); ASI (Italy); MCINN (Spain); Stockholm Observatory (Sweden); STFC (UK); and NASA (USA).

SPIRE has been developed by a consortium of institutes led by Cardiff Univ. (UK) and including: Univ. Lethbridge (Canada); NAOC (China); CEA, LAM (France); IFSI, Univ. Padua (Italy); IAC (Spain); Stockholm Observatory (Sweden); Imperial College London, RAL, UCL-MSSL, UKATC, Univ. Sussex (UK); and Caltech, JPL, NHSC, Univ. Colorado (USA). This development has been supported by national funding agencies: CSA (Canada); NAOC (China); CEA, CNES, CNRS (France); ASI (Italy); MCINN (Spain); SNSB (Sweden); STFC, UKSA (UK); and NASA (USA).

The research leading to these results has received funding from the European Community's Seventh Framework Programme (/FP7/2007-2013/) under grant agreement No 22951 


\section{REFERENCES}

Aloisi, A., van der Marel, R. P., Mack, J., et al. 2005, ApJ, 631, L45

Aloisi, A., Clementini, G., Tosi, M., et al. 2007, ApJ, 667, L151

Aniano, G., Draine, B. T., Calzetti, D., et al. 2012, ArXiv e-prints

Asplund, M., Grevesse, N., Sauval, A. J., \& Scott, P. 2009, ARA\&A, 47, 481

Bakes, E. L. O., \& Tielens, A. G. G. M. 1994, ApJ, 427, 822

Bayet, E., Gerin, M., Phillips, T. G., \& Contursi, A. 2004, A\&A, 427, 45

-. 2006, A\&A, 460, 467

—. 2009, MNRAS, 399, 264

Begum, A., \& Chengalur, J. N. 2005, MNRAS, 362, 609

Bendo, G. J., Galliano, F., \& Madden, S. C. 2012a, arXiv1202.4629B

Bendo, G. J., Buckalew, B. A., Dale, D. A., et al. 2006, ApJ, 645, 134

Bendo, G. J., Wilson, C. D., Pohlen, M., et al. 2010, A\&A, 518, L65

Bendo, G. J., Boselli, A., Dariush, A., et al. 2012b, MNRAS, 419, 1833

Bergvall, N., Masegosa, J., Östlin, G., \& Cernicharo, J. 2000, A\&A, 359, 41

Bergvall, N., \& Östlin, G. 2002, A\&A, 390, 891

Bergvall, N., Zackrisson, E., Andersson, B.-G., et al. 2006, A\&A, 448, 513

Bettoni, D., Galletta, G., \& García-Burillo, S. 2003, A\&A, 405, 5

Bigiel, F., Leroy, A., Walter, F., et al. 2008, AJ, 136, 2846 
Bigiel, F., Leroy, A. K., Walter, F., et al. 2011, ApJ, 730, L13

Bolatto, A. D., Jackson, J. M., Wilson, C. D., \& Moriarty-Schieven, G. 2000, ApJ, 532, 909

Boquien, M., Calzetti, D., Combes, F., et al. 2011, AJ, 142, 111

Bordalo, V., Plana, H., \& Telles, E. 2009, ApJ, 696, 1668

Boselli, A., Gavazzi, G., Lequeux, J., \& Pierini, D. 2002, A\&A, 385, 454

Boselli, A., Ciesla, L., Buat, V., et al. 2010, A\&A, 518, L61

Boselli, A., Ciesla, L., Cortese, L., et al. 2012, A\&A, 540, A54

Bot, C., Boulanger, F., Lagache, G., Cambrésy, L., \& Egret, D. 2004, A\&A, 423, 567

Böttner, C., Klein, U., \& Heithausen, A. 2003, A\&A, 408, 493

Brauher, J. R., Dale, D. A., \& Helou, G. 2008, ApJS, 178, 280

Cannon, J. M., Dohm-Palmer, R. C., Skillman, E. D., et al. 2003, AJ, 126, 2806

Cannon, J. M., Smith, J.-D. T., Walter, F., et al. 2006, ApJ, 647, 293

Chengalur, J. N., Pustilnik, S. A., Martin, J.-M., \& Kniazev, A. Y. 2006, MNRAS, 371, 1849

Ciesla, L., Boselli, A., Smith, M. W. L., et al. 2012, A\&A, 543, A161

Cormier, D., Madden, S. C., Hony, S., et al. 2010, A\&A, 518, L57+

Cormier, D., Lebouteiller, V., Madden, S. C., et al. 2012, ArXiv e-prints

Dale, D. A., Aniano, G., Engelbracht, C. W., et al. 2012, ApJ, 745, 95

Dale, D. A., \& Helou, G. 2002, ApJ, 576, 159 
Davies, J. I., Bianchi, S., Cortese, L., et al. 2012, MNRAS, 419, 3505

Davoust, E., \& Contini, T. 2004, A\&A, 416, 515

de Looze, I., Baes, M., Bendo, G. J., Cortese, L., \& Fritz, J. 2011, MNRAS, 416, 2712

De Looze, I., Baes, M., Parkin, T. J., et al. 2012, MNRAS, 423, 2359

de Vaucouleurs, G., de Vaucouleurs, A., Corwin, Jr., H. G., et al. 1991, Third Reference Catalogue of Bright Galaxies. Volume I: Explanations and references. Volume II: Data for galaxies between $0^{h}$ and $12^{h}$. Volume III: Data for galaxies between $12^{h}$ and $24^{h}$.

Draine, B. T., Dale, D. A., Bendo, G., et al. 2007, ApJ, 663, 866

Draine, B. T., \& Hensley, B. 2012, ArXiv e-prints

Draine, B. T., \& Lazarian, A. 1998, ApJ, 494, L19+

Draine, B. T., \& Li, A. 2007, ApJ, 657, 810

Dwek, E. 1998, ApJ, 501, 643

Edmunds, M. G. 2001, MNRAS, 328, 223

Ekta, B., \& Chengalur, J. N. 2010, MNRAS, 403, 295

Ekta, B., Pustilnik, S. A., \& Chengalur, J. N. 2009, MNRAS, 397, 963

Engelbracht, C. W., Rieke, G. H., Gordon, K. D., et al. 2008, ApJ, 678, 804

Engelbracht, C. W., et al. 2005, ApJ, 628, L29

Eskew, M., Zaritsky, D., \& Meidt, S. 2012, AJ, 143, 139

Fanelli, M. N., Waller, W. W., Smith, D. A., et al. 1997, ApJ, 481, 735 
Foyle, K., Wilson, C. D., Mentuch, E., et al. 2012, MNRAS, 421, 2917

Fricke, K. J., Izotov, Y. I., Papaderos, P., Guseva, N. G., \& Thuan, T. X. 2001, AJ, 121, 169

Fritz, J., Gentile, G., Smith, M. W. L., et al. 2012, A\&A, 546, A34

Galametz, M., Madden, S., Galliano, F., et al. 2009, A\&A, 508, 645

Galametz, M., Madden, S. C., Galliano, F., et al. 2010, A\&A, 518, L55+

-. 2011, A\&A, 532, A56

Galametz, M., Kennicutt, R. C., Albrecht, M., et al. 2012, ArXiv e-prints

Galliano, F., Dwek, E., \& Chanial, P. 2008, ApJ, 672, 214

Galliano, F., Hony, S., Bernard, J.-P., et al. 2011, A\&A, 536, A88

Galliano, F., Madden, S. C., Jones, A. P., et al. 2003, A\&A, 407, 159

-. 2005, A\&A, 434, 867

Genzel, R., Tacconi, L. J., Combes, F., et al. 2012, ApJ, 746, 69

Gieren, W., Pietrzyński, G., Nalewajko, K., et al. 2006, ApJ, 647, 1056

Gil de Paz, A., \& Madore, B. F. 2005, ApJS, 156, 345

Glover, S. C. O., \& Clark, P. C. 2012, MNRAS, 421, 9

Gordon, D., \& Gottesman, S. T. 1981, AJ, 86, 161

Grenier, I. A., Casandjian, J.-M., \& Terrier, R. 2005, Science, 307, 1292

Griffin, M. J., Abergel, A., Abreu, A., et al. 2010, A\&A, 518, L3+ 
Grocholski, A. J., van der Marel, R. P., Aloisi, A., et al. 2012, AJ, 143, 117

Grossi, M., Hunt, L. K., Madden, S., et al. 2010, A\&A, 518, L52+

Guseva, N. G., Izotov, Y. I., Fricke, K. J., \& Henkel, C. 2012, ArXiv e-prints

Guseva, N. G., Izotov, Y. I., Papaderos, P., \& Fricke, K. J. 2007, A\&A, 464, 885

Guseva, N. G., Izotov, Y. I., \& Thuan, T. X. 2000, ApJ, 531, 776

Guseva, N. G., Papaderos, P., Izotov, Y. I., et al. 2003a, A\&A, 407, 91

-. 2003b, A\&A, 407, 105

Hailey-Dunsheath, S., Nikola, T., Stacey, G. J., et al. 2010, ApJ, 714, L162

Helou, G. 1986, ApJ, 311, L33

Hermelo, I., Lisenfeld, U., Relaño, M., et al. 2013, A\&A, 549, A70

Hoang, T., Lazarian, A., \& Draine, B. T. 2011, ApJ, 741, 87

Hopkins, A. M., Schulte-Ladbeck, R. E., \& Drozdovsky, I. O. 2002, AJ, 124, 862

Houck, J. R., Roellig, T. L., van Cleve, J., et al. 2004, ApJS, 154, 18

Huchtmeier, W. K., Krishna, G., \& Petrosian, A. 2005, A\&A, 434, 887

Hunt, L. K., Thuan, T. X., Izotov, Y. I., \& Sauvage, M. 2010, ApJ, 712, 164

Hunter, D. A., Elmegreen, B. G., \& Ludka, B. C. 2010, AJ, 139, 447

Hunter, D. A., Gallagher, III, J. S., Rice, W. L., \& Gillett, F. C. 1989, ApJ, 336, 152

Hunter, D. A., \& Kaufman, M. 2007, AJ, 134, 721

Hunter, D. A., Zahedy, F., Bowsher, E. C., et al. 2011, AJ, 142, 173 
Hunter, D. A., Kaufman, M., Hollenbach, D. J., et al. 2001, ApJ, 553, 121

Israel, F. P., \& Maloney, P. R. 2011, ArXiv e-prints

Israel, F. P., Maloney, P. R., Geis, N., et al. 1996, ApJ, 465, 738

Issa, M. R., MacLaren, I., \& Wolfendale, A. W. 1990, A\&A, 236, 237

Izotov, I. I., Guseva, N. G., Lipovetskii, V. A., Kniazev, A. I., \& Stepanian, J. A. 1991, A\&A, 247, 303

Izotov, Y. I., Papaderos, P., Guseva, N. G., Fricke, K. J., \& Thuan, T. X. 2004, A\&A, 421, 539

Izotov, Y. I., Stasińska, G., Meynet, G., Guseva, N. G., \& Thuan, T. X. 2006, A\&A, 448, 955

Izotov, Y. I., \& Thuan, T. X. 1998, ApJ, 500, 188

-. 1999, ApJ, 511, 639

Izotov, Y. I., Thuan, T. X., \& Lipovetsky, V. A. 1994, ApJ, 435, 647

-. 1997, ApJS, 108, 1

Izotov, Y. I., Thuan, T. X., \& Stasińska, G. 2007, ApJ, 662, 15

Jackson, D. C., Cannon, J. M., Skillman, E. D., et al. 2006, ApJ, 646, 192

James, A., Dunne, L., Eales, S., \& Edmunds, M. G. 2002, MNRAS, 335, 753

Jones, A. P. 2012a, A\&A, 540, A1

-. 2012b, A\&A, 540, A2

-. 2012c, A\&A, 542, A98 
Kamenetzky, J., Glenn, J., Rangwala, N., et al. 2012, ApJ, 753, 70

Karachentsev, I. D., Karachentseva, V. E., Huchtmeier, W. K., \& Makarov, D. I. 2004, AJ, 127,2031

Karachentsev, I. D., Makarov, D. I., \& Huchtmeier, W. K. 1999, A\&AS, 139, 97

Karachentsev, I. D., Dolphin, A. E., Geisler, D., et al. 2002, A\&A, 383, 125

Karachentsev, I. D., Sharina, M. E., Dolphin, A. E., et al. 2003, A\&A, 398, 467

Karczewski, O. Ł., Barlow, M. J., Page, M. J., et al. 2013, ArXiv e-prints

Kaufman, M. J., Wolfire, M. G., \& Hollenbach, D. J. 2006, ApJ, 644, 283

Kennicutt, R. C., Calzetti, D., Aniano, G., et al. 2011, PASP, 123, 1347

Kennicutt, Jr., R. C. 1998, ApJ, 498, 541

Kennicutt, Jr., R. C., Armus, L., Bendo, G., et al. 2003, PASP, 115, 928

Kennicutt, Jr., R. C., et al. 2007, ApJ, 671, 333

Kewley, L. J., \& Ellison, S. L. 2008, ApJ, 681, 1183

Kim, M., Kim, E., Hwang, N., et al. 2009, ApJ, 703, 816

Kiuchi, G., Ohta, K., Sawicki, M., \& Allen, M. 2004, AJ, 128, 2743

Kobulnicky, H. A., Kennicutt, Jr., R. C., \& Pizagno, J. L. 1999, ApJ, 514, 544

Kobulnicky, H. A., \& Kewley, L. J. 2004, ApJ, 617, 240

Kobulnicky, H. A., \& Skillman, E. D. 1996, ApJ, 471, 211

—. 1997, ApJ, 489, 636 
Kong, X., \& Cheng, F. Z. 2002, A\&A, 389, 845

Koribalski, B. S., Staveley-Smith, L., Kilborn, V. A., et al. 2004, AJ, 128, 16

Krumholz, M. R., \& Gnedin, N. Y. 2011, ApJ, 729, 36

Lebouteiller, V., Barry, D. J., Spoon, H. W. W., et al. 2011, ApJS, 196, 8

Lebouteiller, V., Cormier, D., Madden, S. C., et al. 2012, ArXiv e-prints

Lee, H., \& Skillman, E. D. 2004, ApJ, 614, 698

Lee, H., Skillman, E. D., \& Venn, K. A. 2006, ApJ, 642, 813

Leroy, A. K., Bolatto, A., Gordon, K., et al. 2011, ArXiv e-prints

Lisenfeld, U., \& Ferrara, A. 1998, ApJ, 496, 145

Lisenfeld, U., Israel, F. P., Stil, J. M., \& Sievers, A. 2002, A\&A, 382, 860

López-Sánchez, Á. R., \& Esteban, C. 2010, A\&A, 517, A85

López-Sánchez, Á. R., Koribalski, B. S., van Eymeren, J., et al. 2012, MNRAS, 419, 1051

Lynds, R., Tolstoy, E., O’Neil, Jr., E. J., \& Hunter, D. A. 1998, AJ, 116, 146

MacKenty, J. W., Maíz-Apellániz, J., Pickens, C. E., Norman, C. A., \& Walborn, N. R. 2000, AJ, 120, 3007

Madden, S. C. 2000, New Astronomy Review, 44, 249

Madden, S. C., Galliano, F., Jones, A. P., \& Sauvage, M. 2006, A\&A, 446, 877

Madden, S. C., Poglitsch, A., Geis, N., Stacey, G. J., \& Townes, C. H. 1997, ApJ, 483, 200

Maddox, S. J., Efstathiou, G., Sutherland, W. J., \& Loveday, J. 1990, MNRAS, 243, 692 
Magdis, G. E., Daddi, E., Béthermin, M., et al. 2012, ApJ, 760, 6

Magnelli, B., Saintonge, A., Lutz, D., et al. 2012, A\&A, 548, A22

Magrini, L., \& Gonçalves, D. R. 2009, MNRAS, 398, 280

Maiolino, R., Nagao, T., Grazian, A., et al. 2008, A\&A, 488, 463

Martin, C. L. 1998, ApJ, 506, 222

Masegosa, J., Moles, M., \& Campos-Aguilar, A. 1994, ApJ, 420, 576

Mateo, M. L. 1998, ARA\&A, 36, 435

McCall, M. L., Rybski, P. M., \& Shields, G. A. 1985, ApJS, 57, 1

Meixner, M., Galliano, F., Hony, S., et al. 2010, A\&A, 518, L71+

Melisse, J. P. M., \& Israel, F. P. 1994, A\&A, 285, 51

Mentuch Cooper, E., Wilson, C. D., Foyle, K., et al. 2012, ApJ, 755, 165

Meny, C., Gromov, V., Boudet, N., et al. 2007, A\&A, 468, 171

Moles, M., del Olmo, A., Perea, J., et al. 1994, A\&A, 285, 404

Moll, S. L., Mengel, S., de Grijs, R., Smith, L. J., \& Crowther, P. A. 2007, MNRAS, 382, 1877

Mould, J. R., Huchra, J. P., Freedman, W. L., et al. 2000, ApJ, 529, 786

Oberst, T. E., Parshley, S. C., Nikola, T., et al. 2011, ApJ, 739, 100

O'Halloran, B., Galametz, M., Madden, S. C., et al. 2010, A\&A, 518, L58+

O'Halloran, B., Satyapal, S., \& Dudik, R. P. 2006, ApJ, 641, 795 
Panuzzo, P., Rangwala, N., Rykala, A., et al. 2010, A\&A, 518, L37

Paradis, D., Bernard, J.-P., Mény, C., \& Gromov, V. 2011, A\&A, 534, A118

Paradis, D., Dobashi, K., Shimoikura, T., et al. 2012, A\&A, 543, A103

Parkin, T. J., Wilson, C. D., Foyle, K., et al. 2012, MNRAS, 422, 2291

Paturel, G., Theureau, G., Bottinelli, L., et al. 2003, A\&A, 412, 57

Pilyugin, L. S., \& Thuan, T. X. 2005, ApJ, 631, 231

—. 2007, ApJ, 669, 299

Pineda, J. L., Mizuno, N., Röllig, M., et al. 2012, A\&A, 544, A84

Planck Collaboration, Ade, P. A. R., Aghanim, N., et al. 2011, A\&A, 536, A19

Poglitsch, A., Krabbe, A., Madden, S. C., et al. 1995, ApJ, 454, 293

Poglitsch, A., Waelkens, C., Geis, N., et al. 2010, A\&A, 518, L2+

Popescu, C. C., \& Hopp, U. 2000, A\&AS, 142, 247

Pustilnik, S. A., Kniazev, A. Y., Pramskij, A. G., Ugryumov, A. V., \& Masegosa, J. 2003, A\&A, 409, 917

Pustilnik, S. A., \& Martin, J.-M. 2007, A\&A, 464, 859

Pustilnik, S. A., Martin, J.-M., Huchtmeier, W. K., et al. 2002, A\&A, 389, 405

Rangwala, N., Maloney, P. R., Glenn, J., et al. 2011, ApJ, 743, 94

Reach, W. T., Dwek, E., Fixsen, D. J., et al. 1995, ApJ, 451, 188 
Roehlly, Y., Buat, V., Heinis, S., Moreau, C., \& Gimenez, S. 2011, in Astronomical Society of the Pacific Conference Series, Vol. 442, Astronomical Data Analysis Software and Systems XX, ed. I. N. Evans, A. Accomazzi, D. J. Mink, \& A. H. Rots, 25

Rosenberg, J. L., Ashby, M. L. N., Salzer, J. J., \& Huang, J.-S. 2006, ApJ, 636, 742

Roussel, H., Wilson, C. D., Vigroux, L., et al. 2010, A\&A, 518, L66

Sauvage, M., Thuan, T. X., \& Lagage, P. O. 1997, A\&A, 325, 98

Sauvage, M., Vigroux, L., \& Thuan, T. X. 1990, A\&A, 237, 296

Saviane, I., Ivanov, V. D., Held, E. V., et al. 2008, A\&A, 487, 901

Schruba, A., Leroy, A. K., Walter, F., et al. 2012, AJ, 143, 138

Schulte-Ladbeck, R. E., Hopp, U., Greggio, L., Crone, M. M., \& Drozdovsky, I. O. 2001, AJ, 121, 3007

Sharina, M. E., Karachentsev, I. D., \& Tikhonov, N. A. 1996, A\&AS, 119, 499

Skillman, E. D., Côté, S., \& Miller, B. W. 2003, AJ, 125, 610

Smith, J. D. T., Draine, B. T., Dale, D. A., et al. 2007, ApJ, 656, 770

Smith, M. W. L., Eales, S. A., Gomez, H. L., et al. 2012, ApJ, 756, 40

Smoker, J. V., Davies, R. D., Axon, D. J., \& Hummel, E. 2000, A\&A, 361, 19

Spinoglio, L., Pereira-Santaella, M., Busquet, G., et al. 2012, ApJ, 758, 108

Stacey, G. J., Geis, N., Genzel, R., et al. 1991, ApJ, 373, 423

Stacey, G. J., Hailey-Dunsheath, S., Ferkinhoff, C., et al. 2010, ApJ, 724, 957

Thuan, T. X., Hibbard, J. E., \& Lévrier, F. 2004, AJ, 128, 617 
Thuan, T. X., Izotov, Y. I., \& Lipovetsky, V. A. 1995, ApJ, 445, 108

Thuan, T. X., \& Martin, G. E. 1981, ApJ, 247, 823

Thuan, T. X., Sauvage, M., \& Madden, S. 1999, ApJ, 516, 783

Tosi, M., Sabbi, E., Bellazzini, M., et al. 2001, AJ, 122, 1271

Tully, R. B. 1988, Nearby galaxies catalog

Úbeda, L., Maíz-Apellániz, J., \& MacKenty, J. W. 2007a, AJ, 133, 917

-. 2007b, AJ, 133, 932

Ugryumov, A. V., Engels, D., Pustilnik, S. A., et al. 2003, A\&A, 397, 463

Ugryumov, A. V., Engels, D., Kniazev, A. Y., et al. 2001, A\&A, 374, 907

van Eymeren, J., Marcelin, M., Koribalski, B. S., et al. 2009, A\&A, 505, 105

van Zee, L., \& Haynes, M. P. 2006, ApJ, 636, 214

van Zee, L., Haynes, M. P., Salzer, J. J., \& Broeils, A. H. 1996, AJ, 112, 129

van Zee, L., Westpfahl, D., Haynes, M. P., \& Salzer, J. J. 1998, AJ, 115, 1000

Vennik, J., Hopp, U., \& Popescu, C. C. 2000, A\&AS, 142, 399

Vermeij, R., Peeters, E., Tielens, A. G. G. M., \& van der Hulst, J. M. 2002, A\&A, 382, 1042

Walter, F., Brinks, E., de Blok, W. J. G., et al. 2008, AJ, 136, 2563

Walter, F., Cannon, J. M., Roussel, H., et al. 2007, ApJ, 661, 102

Wolfire, M. G., Hollenbach, D., \& McKee, C. F. 2010, ApJ, 716, 1191

Wolfire, M. G., Tielens, A. G. G. M., \& Hollenbach, D. 1990, ApJ, 358, 116 
Wu, Y., Charmandaris, V., Hao, L., et al. 2006, ApJ, 639, 157

Xilouris, E. M., Tabatabaei, F. S., Boquien, M., et al. 2012, A\&A, 543, A74

Ysard, N., Juvela, M., \& Verstraete, L. 2011, A\&A, 535, A89

Zhu, M., Papadopoulos, P. P., Xilouris, E. M., Kuno, N., \& Lisenfeld, U. 2009, ApJ, 706, 941

Zubko, V., Dwek, E., \& Arendt, R. G. 2004, ApJS, 152, 211

Zubko, V. G., Mennella, V., Colangeli, L., \& Bussoletti, E. 1996, MNRAS, 282, 1321 